\title{
Senegal: Staff Report for the 2010 Article IV Consultation, Fifth Review Under the Policy Support Instrument, Third Review Under the Exogenous Shocks Facility, Request for Waiver of Nonobservance of Performance Criterion, and Modification of Assessment Criterion-Staff Report; Debt Sustainability Analysis; Press Release; Executive Director Statement; Public Information Notice
}

In the context of the 2010 Article IV Consultation, Fifth Review Under the Policy Support Instrument, Third Review Under the Exogenous Shocks Facility, Request for Waiver of Nonobservance of Performance Criterion, and Modification of Assessment Criterion, with Senegal, the following documents have been released and are included in this package:

- $\quad$ The Staff Report for the 2010 Article IV Consultation, Fifth Review Under the Policy Support Instrument, Third Review Under the Exogenous Shocks Facility, Request for Waiver of Nonobservance of Performance Criterion, and Modification of Assessment Criterion, prepared by a staff team of the IMF, following discussions that ended on March 25, 2010, with officials of Senegal on economic developments and policies. Based on information available at the time of these discussions, the staff report was completed on May 7, 2010. The views expressed in the staff report are those of the staff team and do not necessarily reflect the views of the Executive Board of the IMF.

- $\quad$ A joint IMF/IDA Debt Sustainability Analysis

- $\quad$ A Press Release on the completion of the reviews

- $\quad$ A statement by the Executive Director for Senegal

- $\quad$ A Public Information Notice

The documents listed below have been or will be separately released.

Letter of Intent sent to the IMF by the authorities of Senegal*

Memorandum of Economic and Financial Policies by the authorities of Senegal*

Technical Memorandum of Understanding*

*Also included in the Staff Report

The policy of publication of staff reports and other documents allows for the deletion of market-sensitive information.

Copies of this report are available to the public from International Monetary Fund • Publication Services

$70019^{\text {th }}$ Street, N.W. $\bullet$ Washington, D.C. 20431

Telephone: (202) 623-7430 • Telefax: (202) 623-7201

E-mail: publications@imf.org Internet: http://www.imf.org

\section{International Monetary Fund Washington, D.C.}




\title{
INTERNATIONAL MONETARY FUND
}

\section{SENEGAL}

\section{Staff Report for the 2010 Article IV Consultation, Fifth Review Under the Policy Support Instrument, Third Review Under the Exogenous Shocks Facility, Request for Waiver of Nonobservance of Performance Criteria, and Modification of Assessment Criterion}

\author{
Prepared by the African Department \\ (In consultation with other departments) \\ Approved by Roger Nord and Thomas Dorsey
}

May 7, 2010

\begin{abstract}
Article IV consultation and program discussions were held in Dakar during March 1125, 2010. The team comprised Mr. Funke (head), Mr. Lakwijk, Mr. Gitton (all AFR), Ms. Sancak (FAD), Mr. Painchaud (SPR), and Ms. Fichera (resident representative). Mr. Sembene (OED) joined the policy discussions. The team met with Finance Minister Diop, Budget Minister Diop, Agriculture Minister Sarr, Energy Minister Sarr, International Cooperation and Infrastructure Minister Wade, BCEAO National Director Diop, other senior government officials, and representatives of development partners and the private sector. The mission cooperated closely with the World Bank.
\end{abstract}

Seminars and outreach. In two half-day seminars the authorities presented the findings of their studies on (i) competitiveness in the West African Economic and Monetary Union (WAEMU); (ii) intertemporal budget constraints and their impact on growth; and (iii) the impact on growth of the state's arrears to the private sector. The team made presentations on (i) liquidity management; (ii) fiscal risks and debt sustainability; (iii) competitiveness of the Senegalese economy; (iv) the role of the banking sector in government financing; and (v) the revised debt sustainability framework. The mission met with representatives of parliament, trade unions, and civil society organizations.

Article IV consultations. The discussions centered on how to regain sustained economic growth after the external and domestic shocks of the past two years.

Fifth PSI and third ESF reviews. Staff recommends their completion. All but one quantitative assessment/performance criteria have been met and most structural conditionality has been observed. Staff supports the authorities' request for waivers for nonobservance of the assessment/performance criterion on the fiscal balance because of corrective actions taken by the authorities.

Exchange restrictions and regime. Senegal, a member of the WAEMU, accepted the obligations under Article VIII, Sections 2(a), 3 and 4 of the Fund's Articles of Agreement as of June 1, 1996, and maintains an exchange system free of restrictions on the making of payments and transfers for current international transactions. The WAEMU's exchange regime is a conventional peg to the euro. 


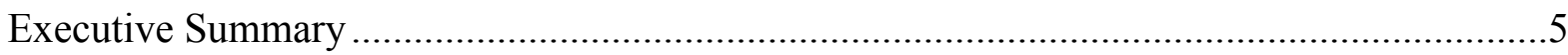

I. Recent Developments and Short-term Outlook: Economic Activity May

Have Bottomed Out

II. Program Performance..............................................................................................

III. Policy Discussions: Regaining Growth Momentum............................................... 9

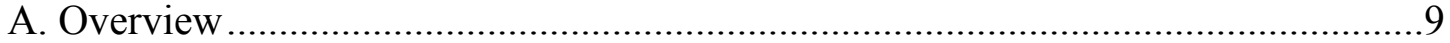

B. Gradually Reducing Fiscal Stimulus .......................................................... 13

C. Fully Normalizing Relations with the Private Sector.......................................... 14

D. Reaping the Benefits of Public Investment....................................................... 14

E. Boosting Competitiveness and Growth Through Accelerated Structural Reforms.16

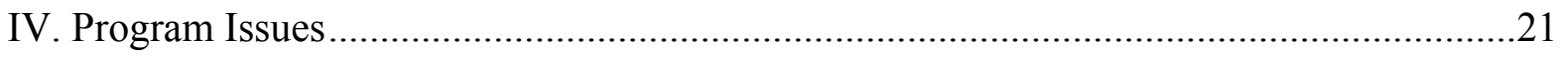

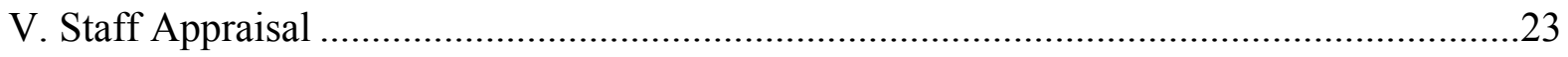

Tables

1. Selected Economic and Financial Indicators, 2007-15 ............................................25

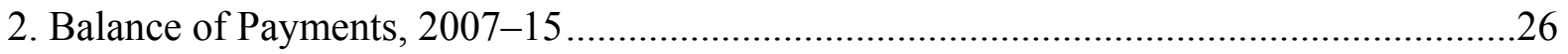

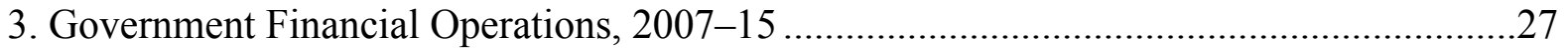

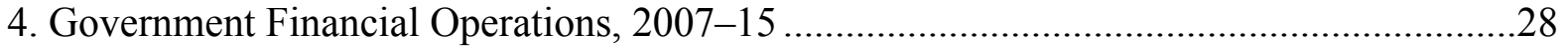

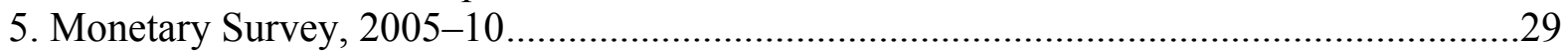

6. Financial Soundness Indicators for the Banking Sector, 2002-09 ..................................30

7. Quantitative Assessment Criteria (Performance Criteria) and Indicative Targets

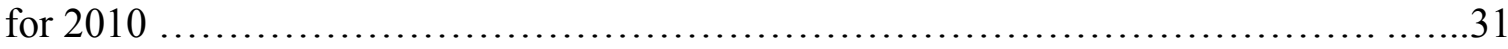

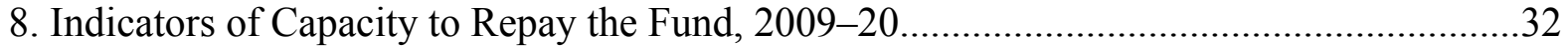

9. Millennium Development Goals............................................... 33

Figures

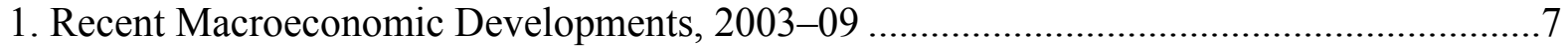

2. Comparing Macroeconomic Projections...................................................................... 10

3. Senegal, WAEMU, and SSA: Macroeconomic Developments and Outlook, 2004-10 ......11

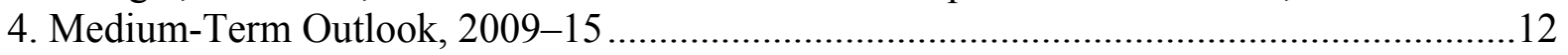

5. Exchange Rate and Structural Competitiveness.................................... 18

6. Financial Sector Issues........................................................... 20

Boxes

1. Senegal's Reactions to Past IMF Advice....................................... 9

2. Extrabudgetary Spending and Public Institution/Agency Debt....................... 15

3. Assessment of the REER and External Competitiveness........................... 17

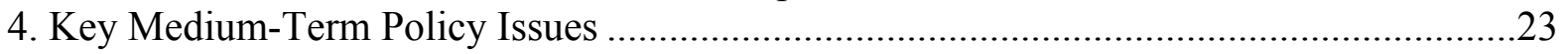


Appendix I. Letter of Intent 34

Attachment I. Memorandum of Financial Policies ................................................................36

Attachment II. Technical Memorandum of Understanding ................................................50 


\section{List of Acronyms}

\begin{tabular}{|c|c|}
\hline AFD & French development agency \\
\hline AIDB & Airport project company \\
\hline ARMP & Procurement regulatory authority \\
\hline ASTER & Expenditure accounting system \\
\hline BCEAO & Central Bank of West African States \\
\hline BC-WAMU & Banking Commission of the West African Monetary Union \\
\hline CIRR & Commercial interest reference rate \\
\hline CPIA & Country Policy and Institutional Assessment \\
\hline CFAF & CFA Francs \\
\hline DCMP & Central public procurement directorate \\
\hline DSA & Debt Sustainability Analysis \\
\hline DGID & Taxes, land and property general directorate \\
\hline $\mathrm{ECF}$ & Extended Credit Facility \\
\hline ESF & Exogenous Shocks Facility \\
\hline FDI & Foreign Direct Investment \\
\hline FNR & Civil servants pension scheme \\
\hline FSIPP & Specific surcharge on petroleum products \\
\hline FSAP & Financial Sector Assessment Program \\
\hline GDP & Gross Domestic Product \\
\hline ICS & Phosphoric acid production company \\
\hline IGF & Office of the Inspector General of Finance \\
\hline $\mathrm{MCA}$ & Millennium Challenge Account \\
\hline MDG & Millennium Development Goals \\
\hline MDRI & Multilateral Debt Relief Initiative \\
\hline MEFP & Memorandum of Economic and Financial Policies \\
\hline NFA & Net Foreign Assets \\
\hline NIR & Net International Reserves \\
\hline NPV & Net present value \\
\hline NPL & Non-performing loan \\
\hline $\mathrm{PC}$ & Performance criterion \\
\hline PEFA & Public Expenditure and Fiscal Accountability \\
\hline PFM & Public financial management \\
\hline PPP & Public Private Partnership \\
\hline PRGF & Poverty Reduction and Growth Facility \\
\hline PRSP & Poverty Reduction Strategy Paper \\
\hline PSI & Policy Support Instrument \\
\hline REER & Real effective exchange rate \\
\hline SAR & Oil refinery company, partly government-owned \\
\hline SDR & Special Drawing Rights \\
\hline SENELEC & Government-owned electricity company \\
\hline SIGFIP & Integrated public financial management system \\
\hline SSA & Sub-Saharan Africa \\
\hline TMU & Technical Memorandum of Understanding \\
\hline TOFE & State financial operations table \\
\hline VAT & Value-added tax \\
\hline WAEMU & West African Economic and Monetary Union \\
\hline
\end{tabular}




\section{Executive Summary}

The external and domestic shocks of the past few years contributed to a major loss in output. Senegal needs to accelerate structural reforms to regain its growth momentum. In a difficult environment, program performance has been mixed on the macroeconomic front but satisfactory on the structural side.

Signs of an emerging recovery: Recent indicators of activity, credit growth, and government revenues suggest that growth may have bottomed out. Real GDP growth, which averaged about 2 percent in 2008 and 2009, is expected to pick up to close to $3 \frac{1}{2}$ percent in 2010. Consumer price inflation, which was negative in the second half of 2009 , is expected to return gradually to its long-run trend of about 2 percent.

Raising growth as a policy priority: The oil, food, and financial crises, coupled with domestic shocks, have contributed to a significant output loss. Improving nonprice competitiveness will be crucial to regaining growth momentum. Authorities and staff agreed that Senegal must accelerate structural reforms in the business climate, governance, the energy sector, and the financial sector to boost competitiveness and growth. In line with findings for the WAEMU, three different approaches suggest that the real effective exchange rate may be modestly overvalued but the estimates are within the normal margin of error.

Broadly satisfactory program performance: All but one quantitative assessment/performance criteria were met. The missed criterion on the fiscal balance points to continued weaknesses in public financial management. Structural reforms were generally satisfactory. With the authorities' firm commitment to further public financial management reforms, on balance staff recommends completion of the fifth PSI and the third and last ESF reviews and granting waivers of nonobservance for the assessment/performance criterion on the fiscal balance because of corrective actions taken by the authorities.

Persistent risks: Risks to growth and the program relate to unexpectedly subdued external demand, financing constraints that limit the fiscal room for maneuver, and renewed problems with electricity supply. Opportunistic changes in economic policies for political reasons could also dampen prospects. 


\section{ReCEnt DeVelopments And Short-term OUtlook: ECONOMIC ACTIVITy MAY HaVe BOTTOMED OUT}

1. Following the food and fuel price shocks in 2008, economic activity slowed further in 2009 because of the global economic downturn and domestic shocks, including temporary electricity shortages (Figure 1, Tables 1-5).

- $\quad$ Economic growth. Real GDP growth is estimated to have been $1 \frac{1}{2}$ percent in 2009 (Table 1). Lower external demand and downward pressure on remittances, tourism receipts, and foreign direct investment (FDI) have reduced growth. The agricultural sector, which benefited from supportive policies and favorable weather, helped to mitigate the impact of depressed demand at home and abroad on the secondary sector (construction, energy) and particularly the tertiary sector (transport, tourism services).

- Inflation. The drop in crude oil and food prices pushed down consumer prices. Inflation has been negative since the second half of 2009.

- $\quad$ Fiscal balance: The overall fiscal deficit widened to 5.1 percent of GDP in 2009 from 3.7 percent of GDP two years earlier on account of lower tax revenues and higher expenditure.

- $\quad$ Balance of payments. The decline in the 2009 current account deficit reflected mainly lower energy and food imports as export performance (outside the recovering chemicals sector) was subdued (Table 2). The global economic downturn caused FDI to recede. ${ }^{1}$

2. There are signs that economic activity has bottomed out. Real GDP growth is projected to reach $3 \frac{1}{2}$ percent in 2010 . The industrial activity index and credit growth to the economy, which had been declining during most of 2009, started to pick up toward yearend. Inflation should return gradually to 2 percent.

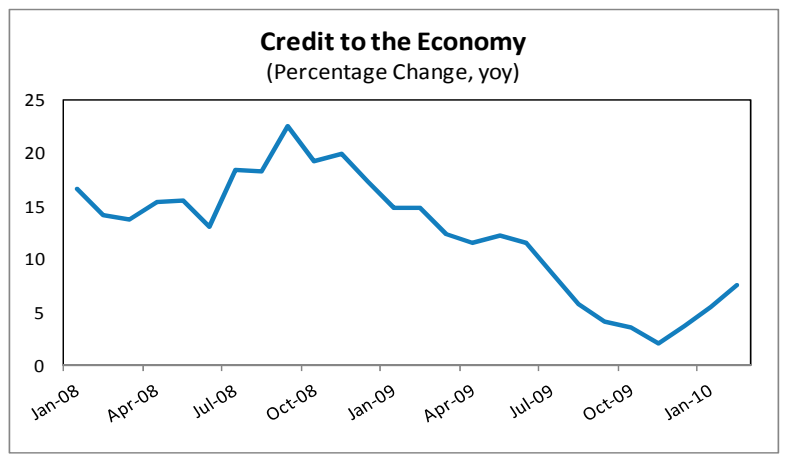

3. Uncertainties about the short-term outlook persist. Risks to growth mainly relate to sluggish external demand, financing constraints that limit the fiscal room for maneuver, and renewed problems with electricity supplies. Opportunistic changes in economic policies for political reasons could also dampen growth prospects. On the positive side, a faster than expected pickup in global activity could have positive spillover effects.

\footnotetext{
${ }^{1}$ Notably, Arcelor-Mittal suspended its US\$2.2 billion projected investment in an iron ore project.
} 
Figure 1. Senegal: Recent Macroeconomic Developments, 2003-09

Economic growth slowed further in 2009 despite progress with the settlement of government payment delays as the world economic crisis affected the country.

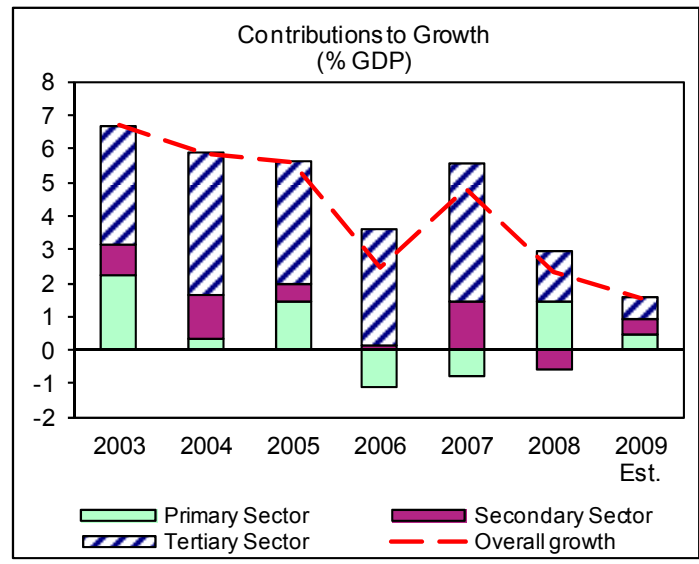

The current account deficit narrowed due to lower imports, partly related to reduced fuel and food prices.

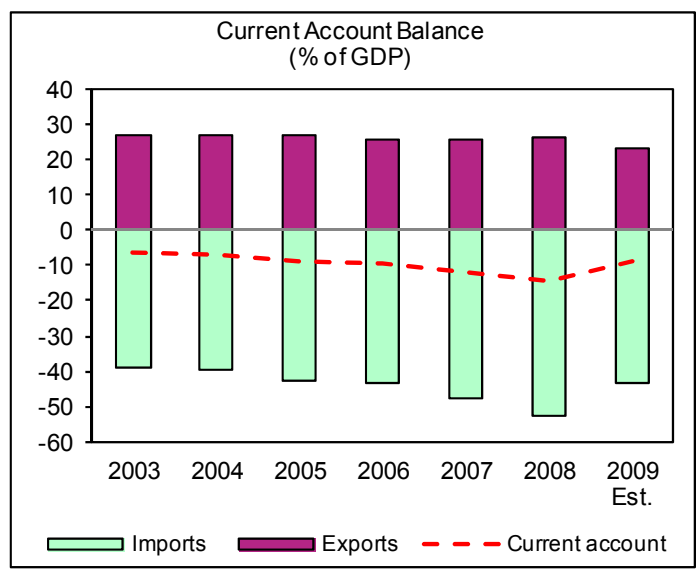

While tax collections came under pressure...

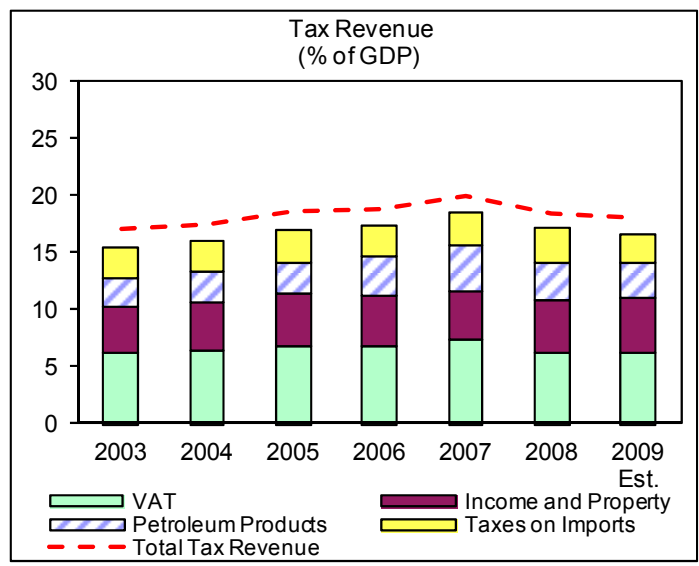

Inflation was negative in the second half of 2009 as food and energy prices declined.

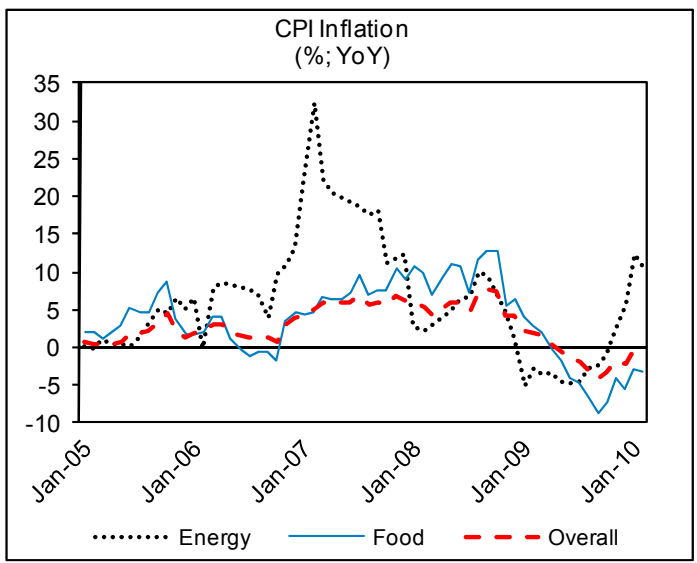

Fiscal policy has been eased in 2008/2009.

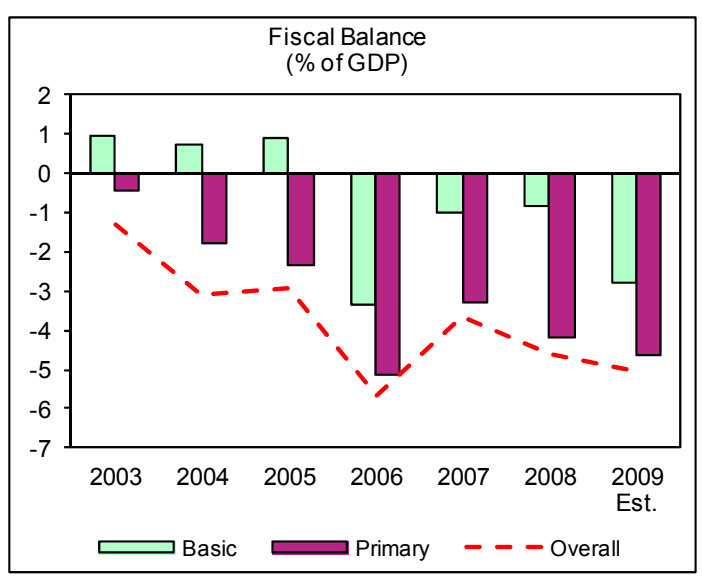

...spending increased.

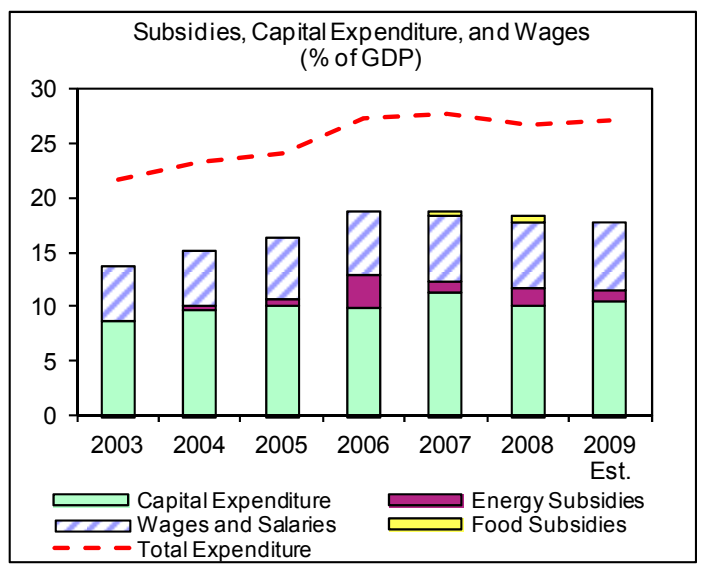

Sources: BCEAO; Senegalese authorities; and IMF staff estimates. 


\section{Program Performance}

4. Macroeconomic performance has been mixed. All quantitative performance/assessment criteria through end-December 2009 except the one for the (basic) fiscal balance were met. Revenues fell short, mostly due to tax arrears of agencies and other public entities. But current expenditure, which was well below program objectives during the first 9 months of 2009, was also higher than programmed (Tables 3 and 4). The authorities explained that they had to pay higher utility bills and use supplemental appropriation decrees (décrets d'avance) totaling some 0.5 percent of GDP, signed by the President and Prime Minister and permitted for emergency measures by the 2001 organic law, including to pay contractuals in the education sector. As a result, the end-2009 basic fiscal deficit was $3 / 4$ percent of GDP higher than targeted (Table 7). While staff appreciated the authorities' increased financial monitoring of public entities in 2010 in view of their tax arrears, staff stressed that the authorities should budget sufficient amounts for essential spending and should have reduced other non-priority expenditure to maintain the budget deficit target. To minimize the risks of higher than initially budgeted current spending, the authorities expressed their intention to offset future supplemental expenditures. This year's budget, for the first time, has a contingency reserve for unforeseen current expenditure. These actions increase the authorities' spending control and form the basis for the waiver request for the missed end-December 2009 performance/assessment criterion.

\section{Performance on the structural side has been satisfactory (Text} Table 1) and structural benchmarks for the fifth review have generally been met. The authorities have issued a press statement on the MEFP $\mid 5$ settlement of extrabudgetary commitments, but some details of the modalities still need to be spelled out. They also elaborated action plans to promote the financial sector and reduce tax expenditures. Planned structural reforms focus on public financial management, settlement of remaining extrabudgetary commitments and agency debt, and tax policy.

Text Table 1. Structural Benchmarks, December 2009-March 2010

\begin{tabular}{lll}
\hline Policy Measures & Target Date & Status \\
\hline $\begin{array}{l}\text { 1. Stop issuing } 2009 \text { payment orders. } \\
\begin{array}{l}\text { 2. Issue a press release summarizing the results of the audit of } \\
\text { extrabudgetary commitments and agency debt, the government's strategy } \\
\text { for settling payments in the first supplementary budget for 2010 submitted } \\
\text { to parliament no later than May 15, 2010, and the settlement procedures. }\end{array}\end{array}$ & January 31, 2010 & $\begin{array}{l}\text { Partly } \\
\text { met }\end{array}$ \\
$\begin{array}{l}\text { 3. Draw up an action plan to suppress tax expenditures with a significant } \\
\text { budgetary impact that are ineffective or inefficient, with the aim of } \\
\text { incorporating the first changes in the 2011 budget. }\end{array}$ & March 31, 2010 & Met \\
\begin{tabular}{l} 
4. Prepare consolidated action plan to implement FSAP recommendations. \\
\hline
\end{tabular} & March 31, 2010 & Met \\
\hline
\end{tabular}




\section{Policy Discussions: Regaining Growth Momentum}

\section{A. Overview}

6. Article IV consultation discussions centered on how to return to higher growth and reduce vulnerabilities to shocks. At the time of the last Article IV consultation, medium-term economic growth was expected to average about $5 \frac{1}{2}$ percent, but annual growth averaged only about 2 percent for 2008-2009 which likely implies a permanent output loss (Figure 2). While Senegal has put in place policies that are consistent with past staff advice (Box 1), structural reform has proved more challenging in part because of the difficult external environment. Progress towards the MDGs has been mixed (Table 9). The successive shocks have complicated the implementation of the authorities' poverty reduction strategy and possibly reversed earlier gains in poverty reduction. While the recent period has shown the limits of existing institutions and policies to help the poor cope with such shocks, the authorities have reaffirmed their commitment to achieve the PRS long-term objectives through the implementation of their reform agenda and higher growth. Work on their PRSP-3 is ongoing and will likely be finalized by the end of the year. Higher growth will be vital if Senegal is to reduce poverty and continue to make significant progress toward the MDGs.

\section{Box 1. Senegal's Reactions to Past IMF Advice}

Senegal and the IMF have a very good working relationship. The country's policies have been generally consistent with IMF recommendations, as reflected in the completion of past ECF (formerly known as PRGF) arrangements and the first four PSI reviews. Authorities and staff generally agree on the main bottlenecks to higher growth. The authorities aim to create a more investment-friendly business environment to foster private sector development.

The authorities acted upon the policy recommendations, particularly in the fiscal area, from the 2008 Article IV consultation and subsequent technical assistance. As was recommended, they corrected much of the fiscal slippages. To reduce the risk of future slippages, the authorities have applied their budget framework more rigorously. In line with the PSI-supported program commitments, they have substantially reduced unpaid bills to the private sector and launched far-reaching PFM reforms. But progress in some structural reforms, outside the Fund's core expertise and not covered by benchmarks, including related to the energy sector and the business climate, has been mixed.

7. Staff and authorities agreed that several complementary policies need to be put in place to regain Senegal's growth momentum and return to previous growth trajectories (Figures 3 and 4): (i) maintaining supportive macroeconomic policies; (ii) fully and expeditiously normalizing relations with the private sector; and (iii) accelerating structural reforms to overcome such bottlenecks as cumbersome business procedures, governance issues, frequent electricity outages, and limited access to finance. 
Figure 2. Senegal: Comparing Macroeconomic Projections

The short-term decline in real GDP growth...

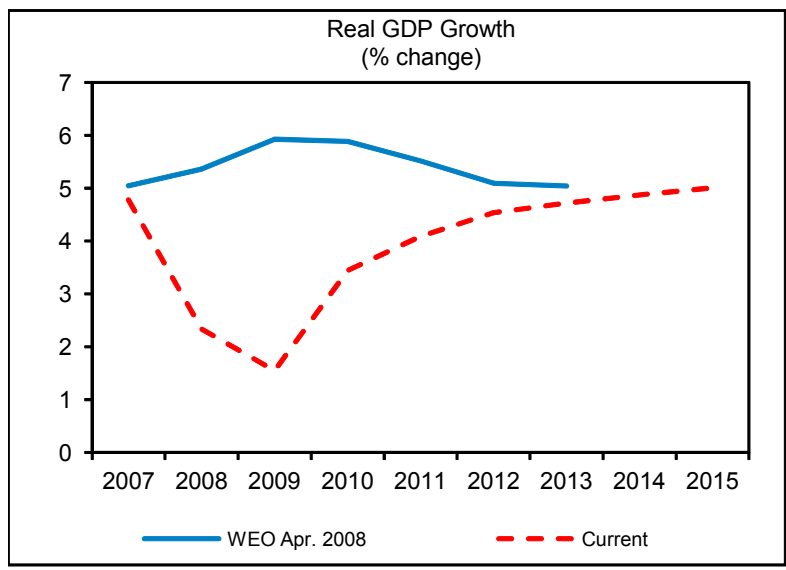

With lower commodity prices, price level projections return to pre-crisis levels.

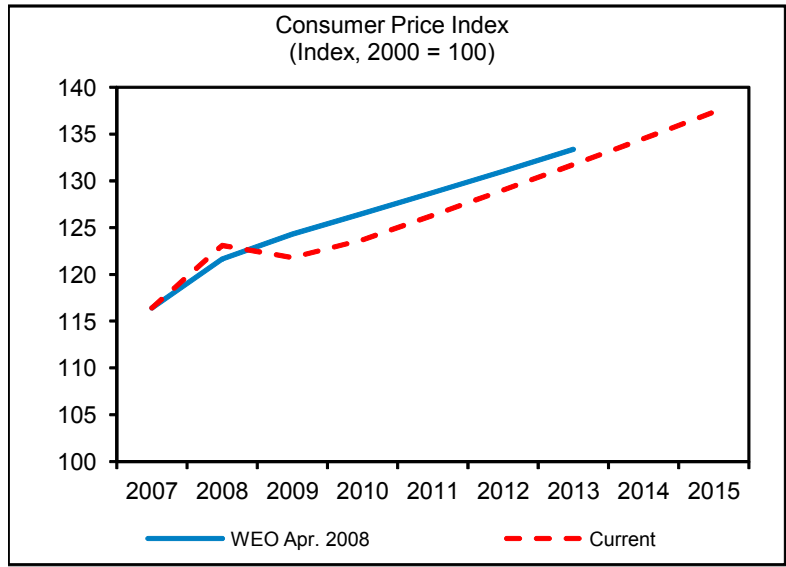

Deficit financing and resource mobilization to settle payment delays increased debt.

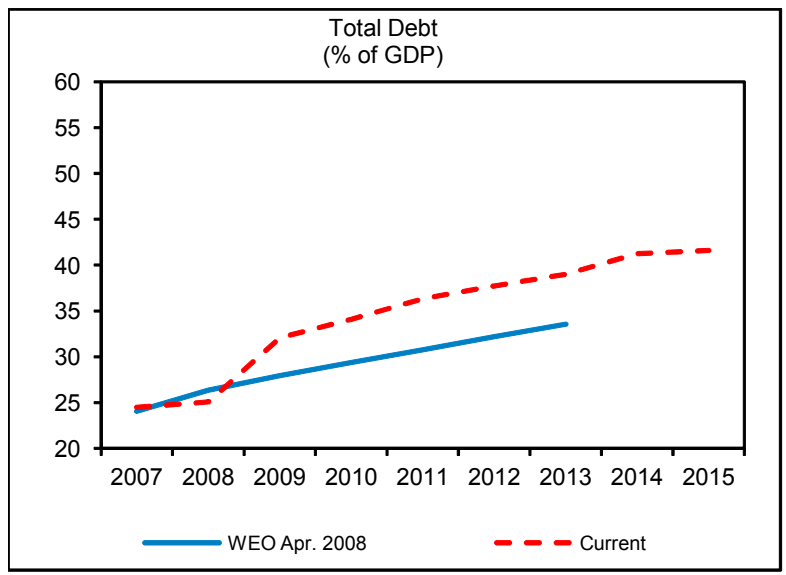

Sources: BCEAO; Senegalese authorities; and IMF staff estimates. ...translates to a significant loss in output.

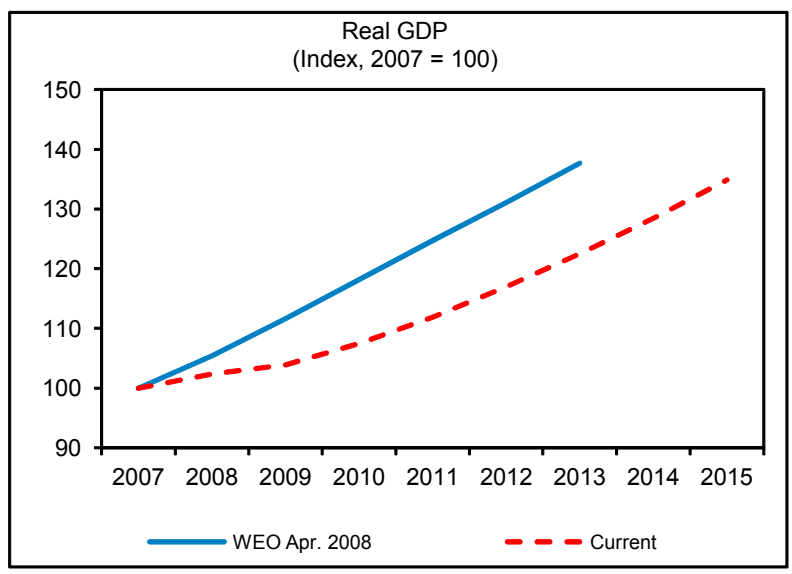

The medium-term fiscal objective remains unchanged.

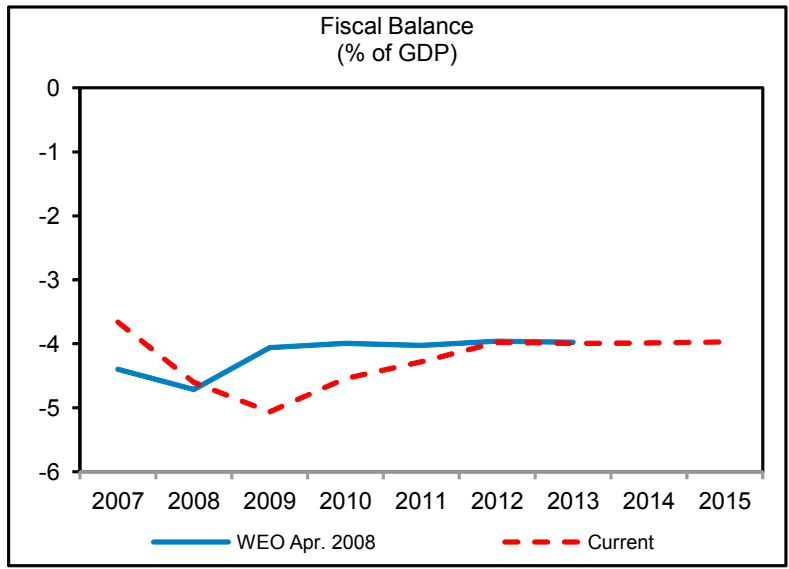

Improved terms of trade lead to a smaller current account deficit than projected earlier.

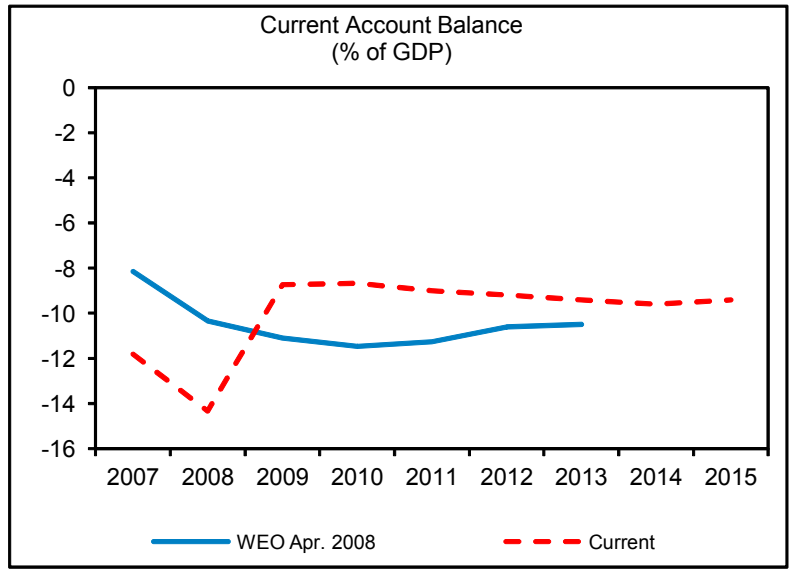


Figure 3. Senegal, WAEMU, and SSA: Macroeconomic Developments and Outlook, 2004-10

Growth has slowed more than in other WAEMU countries...

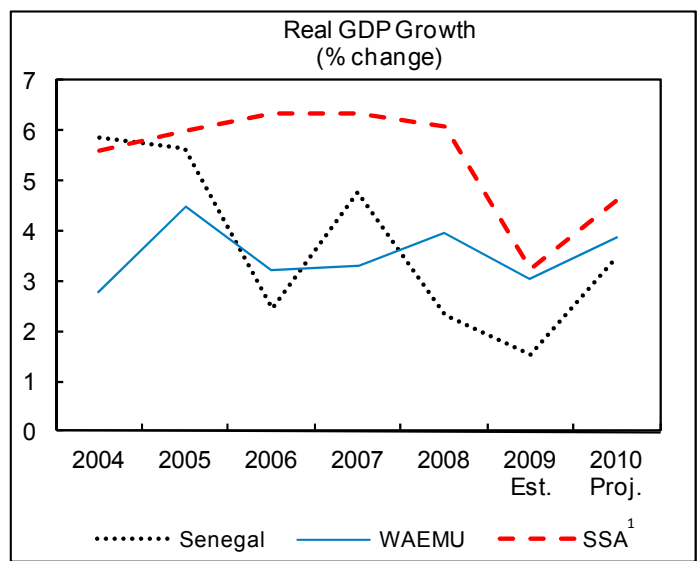

The fiscal deficit has been wider than in the WAEMU ...

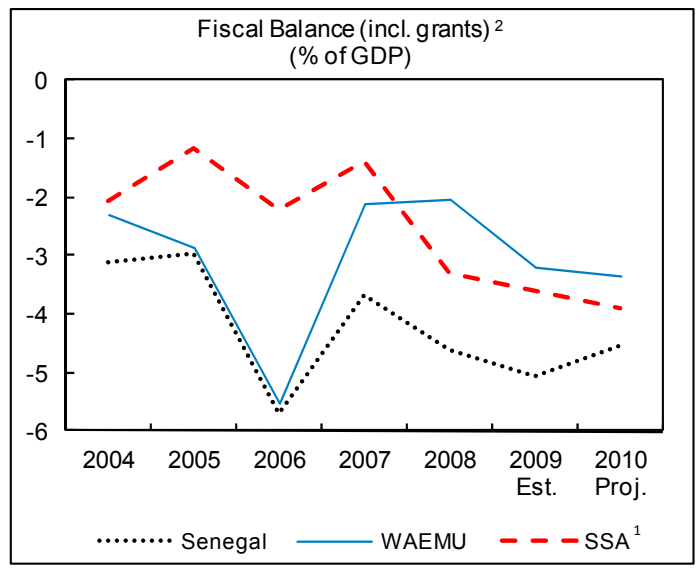

As in the WAEMU, the current account deficit remains large ...

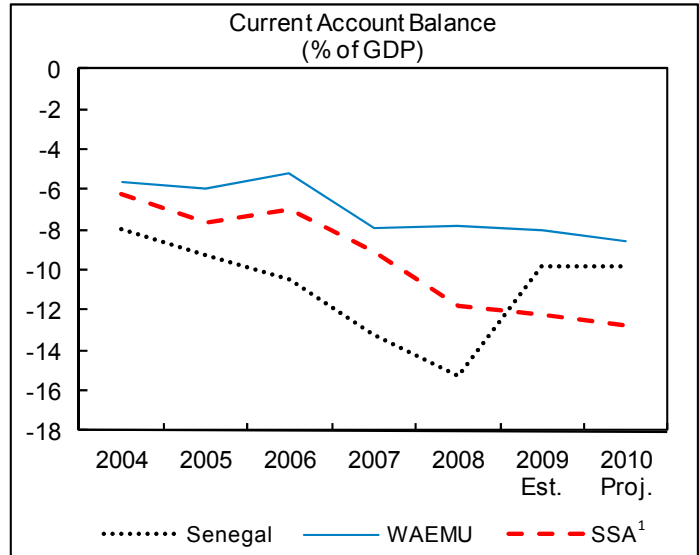

... including markedly in the tertiary sector.

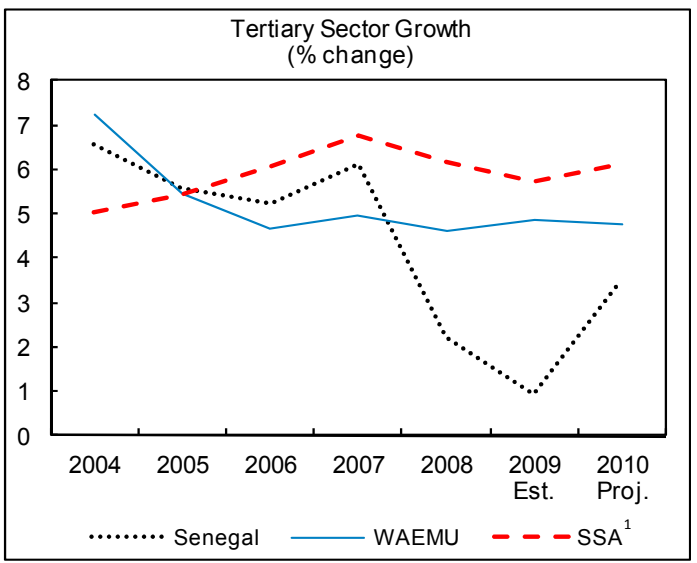

... despite higher government revenue.

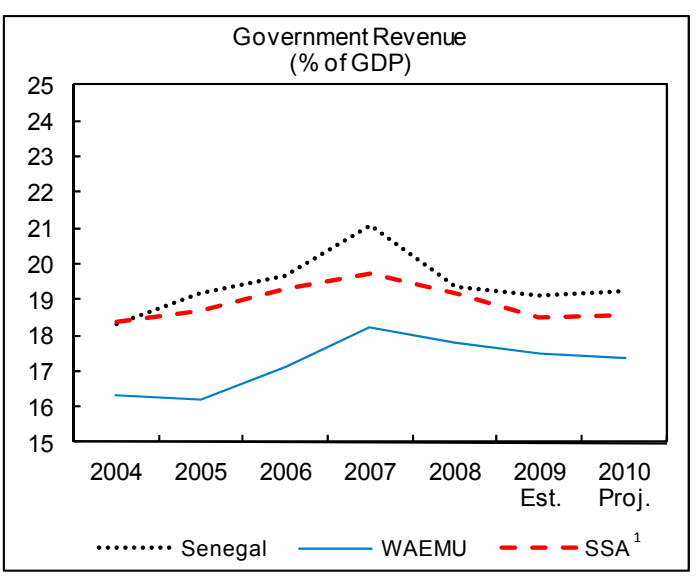

... and FDI inflows are lower than in other SSA economies.

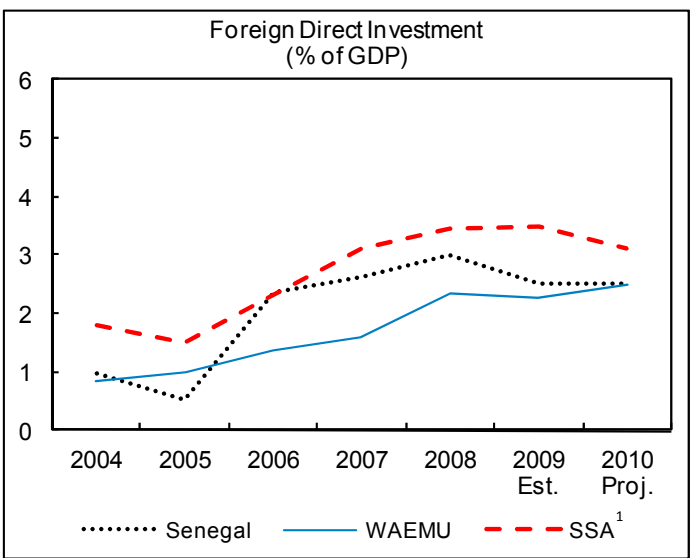

Sources: BCEAO; Senegalese authorities; and IMF staff estimates.

1 Excludes SSA countries classified as Oil-Exporters and South Africa.

${ }^{2}$ For 2006, fiscal balance excluding grants forWAEMU and non-oil SSA to eliminate effect of MDRI relief. 
Figure 4. Senegal: Medium-Term Outlook, 2009-15

Output growth is projected to be driven by the services sector, recovery in ICS' production, and construction related to large investment projects.

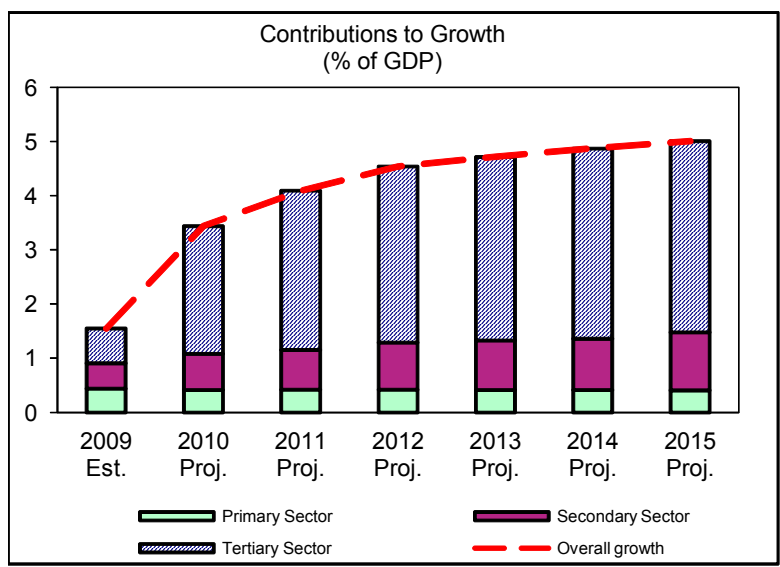

The current account deficit is projected to be financed in part by private flows ...

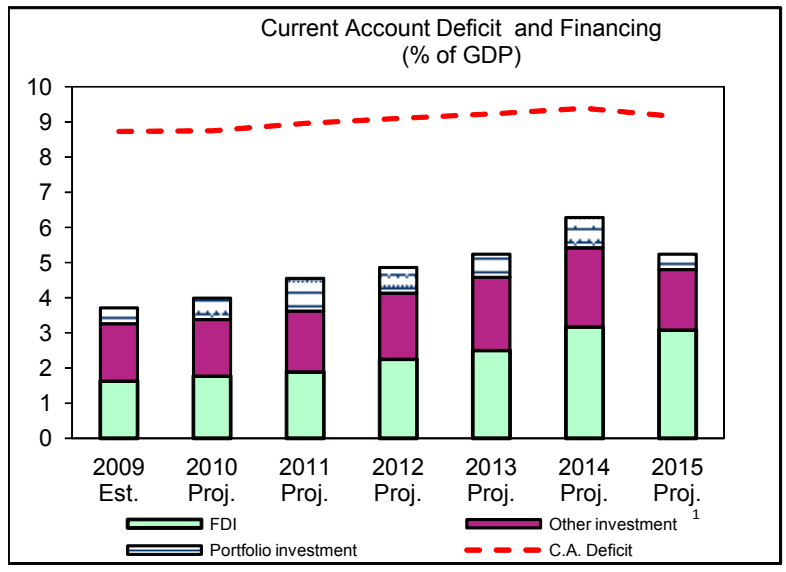

The fiscal balance will be contained...

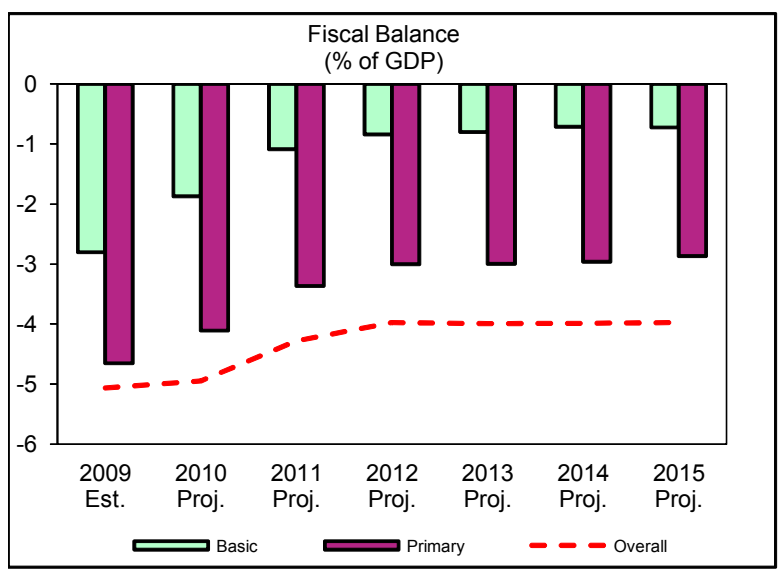

Sources: BCEAO; Senegalese authorities; and IMF staff estimates.

1 Includes only private other investment.
Inflation is projected to return to historical trends in the context of WAEMU membership and in the absence of renewed energy and food price pressures.

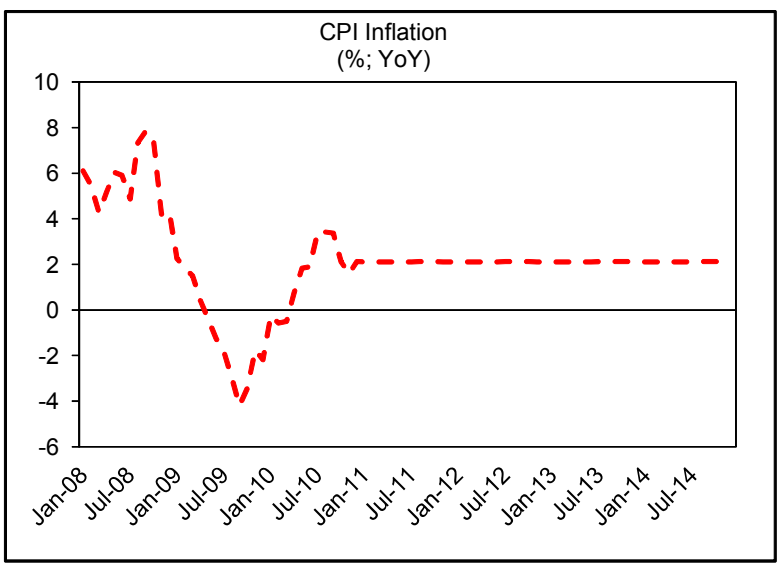

...while the economy remains dependent on foreign assistance.

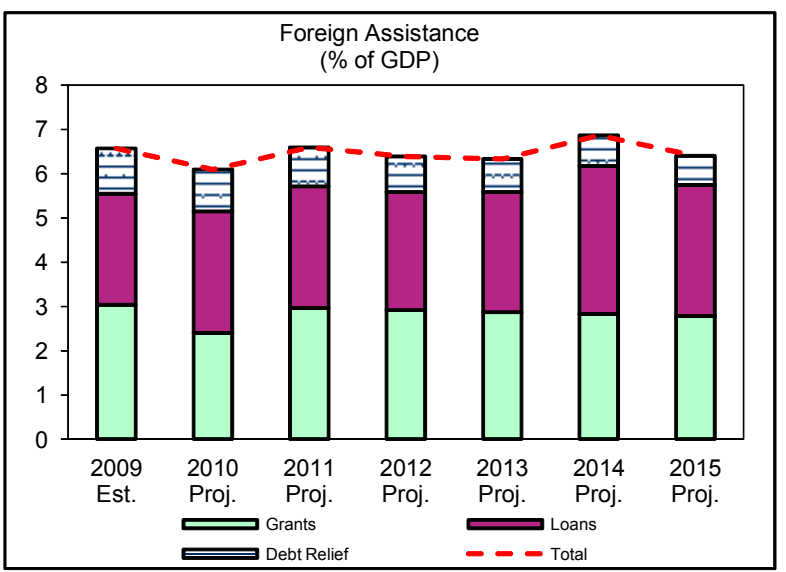

...by controlling current expenditure while over time allowing public investment to rise.

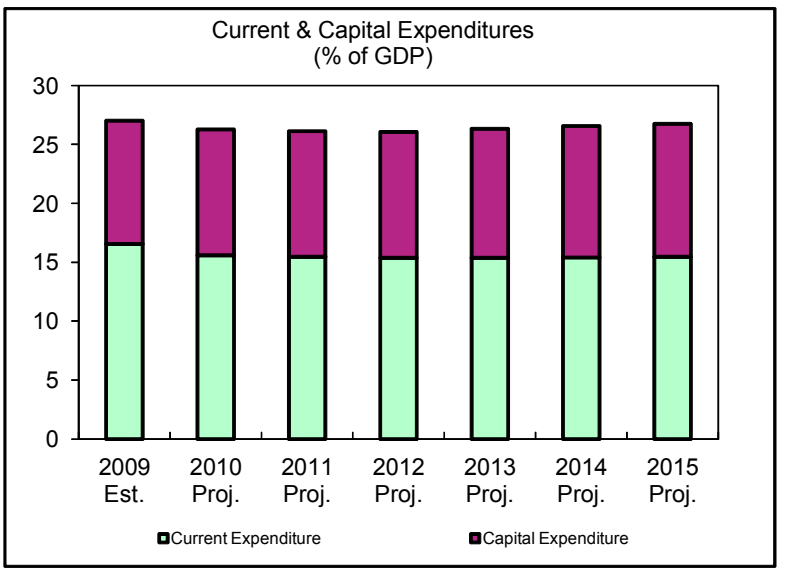




\section{B. Gradually Reducing Fiscal Stimulus}

8. With a higher than expected fiscal deficit in 2009 , financing constraints, and signs of a pick-up in activity, authorities and staff

MEFP $q 8,12$ concurred that the fiscal stimulus should be gradually reduced to progressively achieve a medium-term deficit target of 4 percent of GDP (and somewhat

less in the long run). To contain the deficit, the authorities expressed their commitment to reduce current expenditure and reinforce efforts to bring in more revenue (including through preparing for tax expenditure rationalizationstructural benchmark). To finance the deficit, they plan to tap the regional MEFP $\mid 23$ market several times this year. It appears that tax revenues started to recover in early 2010, and regional liquidity is high. But because of uncertainties about whether these developments will persist, the authorities have agreed to slow expenditure (mostly investment) by about $1 / 2$ percent of GDP. ${ }^{2}$ Authorities and staff concurred that expenditure could be raised back to the budgeted level later in the year, if revenue collection permits or additional financing becomes available through privatization proceeds. To minimize liquidity and financing risks, staff encouraged the authorities to update their treasury and bond issuance plans more often. A gradual reduction in the fiscal deficit will help move Senegal back onto MEFP $\mid 20,21$ the path of meeting WAEMU convergence criteria in the medium term.

Senegal: Compliance with WAEMU Convergence Criteria

\begin{tabular}{|c|c|c|c|c|}
\hline & 2007 & 2008 & 2009 & $\begin{array}{l}2010 \\
\text { Proj. }\end{array}$ \\
\hline \multicolumn{5}{|l|}{ Primary criteria } \\
\hline Basic fiscal balance/GDP ( $\geq 0$ percent) & -1.0 & -0.8 & -2.8 & -1.5 \\
\hline Average inflation $(\leq 3$ percent $)$ & 5.9 & 5.8 & -1.1 & 1.6 \\
\hline Total debt/GDP ( $\leq 70$ percent $)$ & 24.5 & 25.1 & 32.1 & 34.1 \\
\hline Change in domestic arrears $(\leq 0)$ & 0.0 & 0.0 & 0.0 & 0.0 \\
\hline Change in external arrears $(\leq 0)$ & 0.0 & 0.0 & 0.0 & 0.0 \\
\hline \multicolumn{5}{|l|}{ Secondary criteria } \\
\hline Wages and salaries/fiscal revenue ( $\leq 35$ percent) $1 /$ & 28.7 & 30.2 & 32.5 & 32.3 \\
\hline $\begin{array}{l}\text { Domestically financed capital expenditure/fiscal revenue } \\
(\geq 20 \text { percent) }\end{array}$ & 34.4 & 27.3 & 32.9 & 32.9 \\
\hline Current account deficit, excluding grants/GDP $(\leq 5$ percent $)$ & 13.2 & 15.3 & 9.8 & 9.8 \\
\hline Fiscal revenue/GDP ( $\geq 17$ percent $)$ & 21.1 & 19.4 & 18.7 & 19.4 \\
\hline
\end{tabular}

Sources: Senegalese authorities; and Fund staff estimates and projections.

1/ Excluding some contractual wages not classified in the wage bill.

\footnotetext{
${ }^{2}$ Regularization of extrabudgetary spending and public institution/agency debt in the May 2010 supplementary budget continues to be recorded below the line and excluded from the fiscal balance for program purposes (and in Tables 3 and 4) pending finalization of modalities. To the extent that it will eventually be recorded above the line, the fiscal deficit will increase.
} 


\section{Fully Normalizing Relations with the Private Sector}

9. The authorities have made more progress in normalizing financial relations with the private sector than was expected during the last program reviews (Box 2).

Representatives of the private sector confirmed that past payment delays are being addressed. In addition to advancing with the settlement of extrabudgetary spending and public institution and agency debt, the stock of unpaid bills within the regular expenditure chain has been kept at normal levels since mid-2009 (quantitative

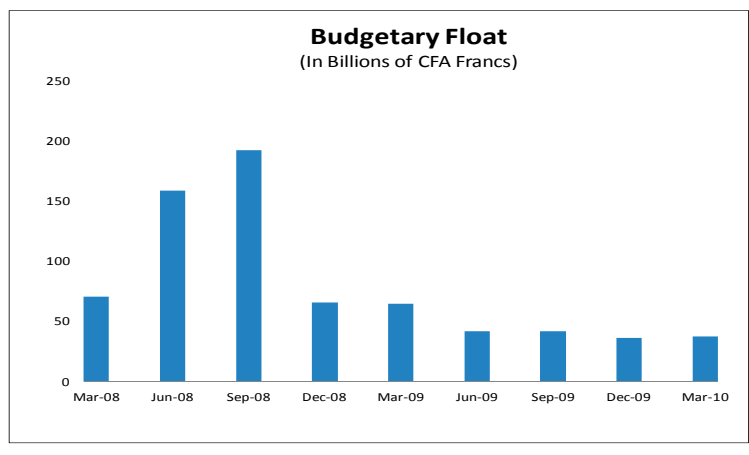
performance / assessment criterion on the budgetary float). ${ }^{3}$ Tight monitoring and management of unpaid bills within the expenditure chain (budgetary float) is important for improving cash flow supervision and preventing budgetary slippages. Staff stressed that liquidity needs to be better managed so that normal fluctuations in the flow of revenues and expenditures can be absorbed without recourse to abrupt spending halts or arrears; to this end, a census of government accounts will be completed (structural benchmark).

\section{The authorities reiterated their commitment to speedily} regularize the remaining extrabudgetary spending and public institution/agency debt through a transparent and publicly

MEFP $q 9,10$ communicated process. This should bring financial relations between the government and the private sector back to normal. The supplementary budget to be submitted to parliament by midMay 2010 will incorporate further regularization, consistent with the authorities' commitment to pay down at least 50 percent of extrabudgetary spending and public institution / agency debt by the end of September 2010 (structural benchmarks). To help prevent a recurrence of payment issues vis-à-vis the private sector, the SIGFIP budget system continues to be improved (structural benchmark), and financial relations between the Treasury and public institutions/agencies have been strengthened.

MEFP $\uparrow 17,18$

\section{Reaping the Benefits of Public Investment}

\section{The authorities are putting more emphasis on public investment to support} economic development and diversification. With the support of donors, the authorities are giving priority to extending and modernizing transport infrastructure to facilitate access to markets. Several large infrastructure projects are progressing, including the Blaise Diagne International Airport, the Dakar-Diamniadio toll road, other road projects, and modernization of the port of Dakar. Plans for other new important projects, such as the establishment of a special economic zone, conversion of the current airport into a business complex, road construction, and water distribution and housing projects, are in various stages of development. The

\footnotetext{
${ }^{3}$ See Box 1 of the last Staff Report (Country Report 10/13).
} 


\section{Box 2. Extrabudgetary Spending and Public Institution/Agency Debt}

The government's financial relations with the private sector are returning to normal as extrabudgetary spending and public institution/agency debt are settled. Of the estimated total of CFAF 119 billion, CFAF 37 billion was found to be intragovernmental (taxes and social security contributions) and to have no impact on the private sector. Out of the remaining total of CFAF 82 billion (1 $1 \frac{1}{2}$ percent of GDP) of extrabudgetary spending and public institution/ agency debt vis-à-vis the private sector, CFAF 35 billion was settled in 2009 and at least half of the remainder will be paid by September 2010.

- $\quad$ Of the extrabudgetary spending by ministeries of CFAF 36 billion (2/3 percent of GDP) that the July 2009 audit identified, some CFAF 10 billion has been regularized in the 2009 and 2010 budgets, and the first supplementary budget for 2010 will regularize the remainder (mid-May structural benchmark).

- $\quad$ Public institution/agency debt to the private sector amounted to CFAF 45 billion ( $3 / 4$ percent of GDP), of which two-thirds was paid in late 2009 when an easing cash position allowed the Treasury to unblock amounts due. The remaining one-third is to be settled in 2010-11.

authorities noted that these projects would support economic diversification, help reduce poverty, ${ }^{4}$ and lessen Senegal's vulnerability to external shocks.

\section{The authorities plan to look at a variety of financing options for their new}

infrastructure projects. They intend to hold a donors' conference on infrastructure financing and seek economic profitability assessments for MEFP $\$ 13,14$ their projects. Following the issuance in December 2009 of a US\$200 million bond with a fiveyear maturity for the Dakar-Diamniadio toll road, the authorities inquired about possible room for additional external nonconcessional financing. Staff stated that nonconcessional borrowing for Senegal should for the time being stay linked to specific projects that have been assessed as economically profitable by a reputable source, for which sufficient concessional financing or financing in the regional market could not be mobilized. Projects must be analyzed case by case. But given Senegal's low risk of debt distress, confirmed by the latest Bank-Fund debt sustainability analysis (DSA), there should in principle be some space for nonconcessional financing for new infrastructure projects as long as they do not jeopardize Senegal's debt sustainability. Untied limits could become possible after PFM improves.

\section{Staff stressed that investment projects should be selected and} prioritized based on rigorous economic cost-benefit analyses. Careful assessments, including analysis of maintenance costs, would make it

\footnotetext{
${ }^{4}$ The livelihood of about half the population is still closely tied to subsistence agriculture.
} 
easier to select the highest-return projects and help raise the productivity of government spending. Progress in this domain could be instrumental in improving Senegal's capacity to manage its finances, for example as reflected in the PEFA (Public Expenditure and Fiscal Accountability) assessment and CPIA (Country Policy and Institutional Assessment).

\section{E. Boosting Competitiveness and Growth Through Accelerated Structural Reforms}

\section{Price and Structural Competitiveness}

\section{Despite a significant devaluation} in 1994, Senegal's export performance has been weak. Its share of world export volumes has been declining gradually since the mid-1980s, though recently production improvements at ICS (phosphoric acid) have slowed the decline.

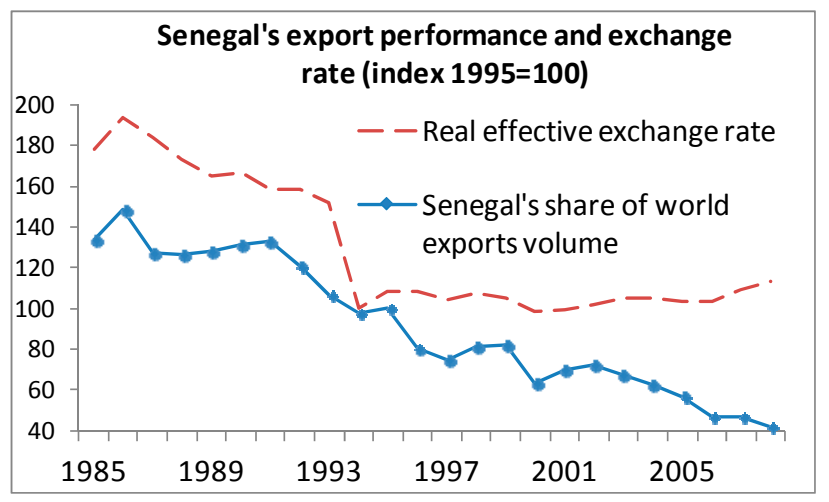

15. While Senegal's real effective exchange rate (REER) has appreciated over the past two years, there is no conclusive evidence that the exchange rate is overvalued. This conclusion is consistent with a recent regional external stability assessment that found the WAEMU region's REER not to be misaligned. It is based on an analysis of possible real exchange rate misalignment undertaken using three complementary approaches (Box 3 and Figure 5). Senegal's competitiveness could be raised by improving the business environment and governance.

MEFP ๆ29-30

\section{Energy Sector Reform}

16. Temporary electricity shortages add to the cost of doing business and fiscal risks. Since 2006 power shortages and blackouts in Senegal have been frequent. The high cost and poor quality of electricity supplies appear to be a major impediment to private investment. ${ }^{5}$ The national electricity company, SENELEC, has been recapitalized in 2007 (CFAF 65 billion) and in 2008 (CFAF 37 billion). But technical failures persist, leading to higher production costs, constraints in fuel supply, delays in putting in place energy-saving measures, tax arrears, and weaknesses in internal governance.

17. Staff stressed that the government's reform plan, in collaboration with the Bank and other donors, needs to be fully and

MEFP q24-26 promptly implemented to limit fiscal and economic risks. The plan builds on a wide range of measures, including restructuring of SENELEC's short-term debt, fresh financing, some tax breaks on new investment, and cost-recovering tariff adjustments. SENELEC will need to be made financially sound on a permanent basis to ensure adequate electricity distribution. Electricity production, transportation, and distribution are expected to be separated in the future.

\footnotetext{
${ }^{5}$ Senegal ranks 113 out of 133 countries for quality of electricity supply in World Economic Forum, Global Competitiveness Report 2009-2010.
} 


\section{Box 3. Assessment of the REER and External Competitiveness}

\section{While Senegal's real effective exchange rate (REER) has appreciated by almost 14 percent since 1994, there is no conclusive evidence that the exchange rate is overvalued, and the recent depreciation of the euro has reduced pressure. An analysis of possible real exchange rate misalignment was undertaken using three complementary approaches:}

Macroeconomic balance: The current account deficit "norm" is estimated at 5.5-6.0 percent of GDP, while the "underlying" current account deficit is estimated at $91 / 4$ percent of GDP, consistent with the projected current account balance over 2012-15. Assuming a trade balance elasticity for small countries of -0.71 , the difference between the norm and the "underlying" current account indicates that the REER is overvalued by 5 percent. $^{2}$

Equilibrium exchange rate: A comparison of the current value of the REER and an estimate of its equilibrium value indicates that the real effective exchange rate is close to its equilibrium. ${ }^{3}$

External sustainability: Assuming a negative net international investment position of 50 percent of GDP, Senegal's current account deficit norm would be about 3.5 percent of GDP. This norm is consistent with a REER overvaluation of about 8 percent. However, the most recent DSA suggests that a larger current account deficit is sustainable given the projected FDI inflows and the concessionality of external financing.

To improve its competitiveness, Senegal needs a better business environment. While its ranking in the Global Competitiveness Report has recently improved, Senegal still ranks 92 out of 133 countries. Areas of weakness the report identified include: (i) higher education and training; (ii) labor market efficiency; (iii) financial market sophistication; (iv) market size; and (v) health and primary education. The World Bank Doing Business Report 2010, in which Senegal ranked 157 out of 183 countries, highlights the need for improvement in the cost of doing business in Senegal in areas such as: (i) hiring workers; (ii) registering property; (iii) protecting investors; and (iv) paying taxes.

Senegal could also improve its competitiveness by enhancing its governance. In the latest CPIA of the World Bank, Senegal ranked 57 out of 75 low-income countries, and areas for improvement are: (i) social protection and labor; (ii) quality of budget and financial management; and (iii) transparency, accountability, and corruption control in the public sector.

\footnotetext{
${ }^{1}$ See Vitek, 2010, Exchange Rate Assessment Toolkit (IMF internal document). The current account norm is based on a panel regression of the current account on a set of fundamentals, including: (i) relative old- age dependency; (ii) relative population growth; (iii) relative income; (iv) relative income growth; (v) oil trade balance; (vi) relative fiscal balance; (vii) initial net foreign assets; (viii) aid flows; and (ix) remittances flows.

${ }^{2}$ See Tokarick, 2009, A Method For Calculating Export Supply and Import Demand Elasticities, mimeo.

${ }^{3}$ See Mongardini and Rayner, 2009, "Grants, Remittances, and the Equilibrium Real Exchange Rate in Sub-Saharan African Countries," IMF Working Paper. The equilibrium value of the REER is based on a panel regression of the REER on a set of fundamentals: (i) terms of trade; (ii) trade openness; (iii) relative productivity; and (iv) relative government consumption.
} 
Figure 5. Senegal: Exchange Rate and Competitiveness

While inflation remained on average close to trading partners...

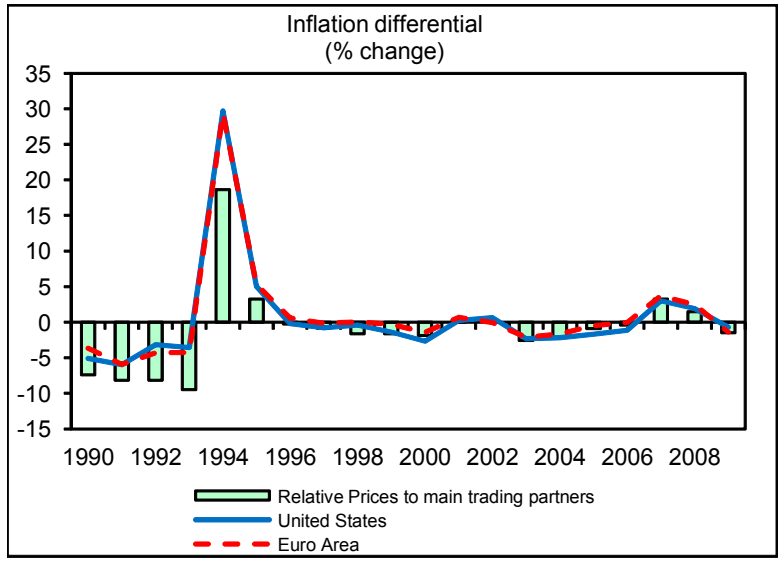

... has contributed to a moderate nominal and real effective exchange rate appreciation...

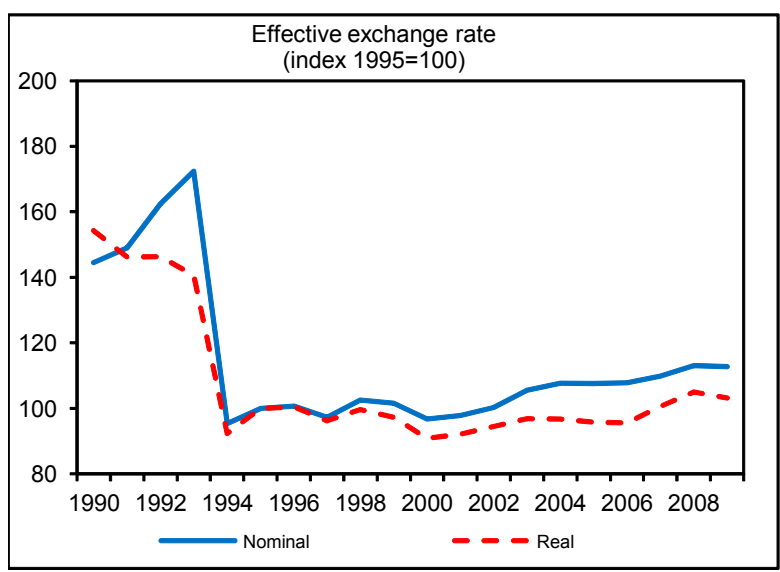

Raising competitiveness rests on enhancing institutions and infrastructure...

\begin{tabular}{|c|c|c|}
\hline \multicolumn{3}{|c|}{ Global Competitiveness Index } \\
\hline & $2008 / 2009$ & $2009 / 2010$ \\
\hline Overall ranking & 96 & 92 \\
\hline Institutions & 83 & 77 \\
\hline o/w Transparency of gov. policy making & 118 & 121 \\
\hline o/w Diversion of public funds & 122 & 114 \\
\hline Infrastructure & 83 & 80 \\
\hline o/w Quality of electricity supply & 118 & 113 \\
\hline o/w Quality of roads & 78 & 78 \\
\hline $\mathrm{o} / \mathrm{w}$ Quality of railroad infrastructure & 93 & 89 \\
\hline Health and primary education & 109 & 108 \\
\hline $\mathrm{o} / \mathrm{w}$ Primary enrollment & 120 & 122 \\
\hline Labor market efficiency & 120 & 110 \\
\hline o/w Rigidity of employment & 118 & 121 \\
\hline Financial market sophistication & 111 & 110 \\
\hline $\mathrm{o} / \mathrm{w}$ Ease of access to loans & 133 & 129 \\
\hline Number of countries & 134 & 133 \\
\hline
\end{tabular}

... the appreciation of the euro...

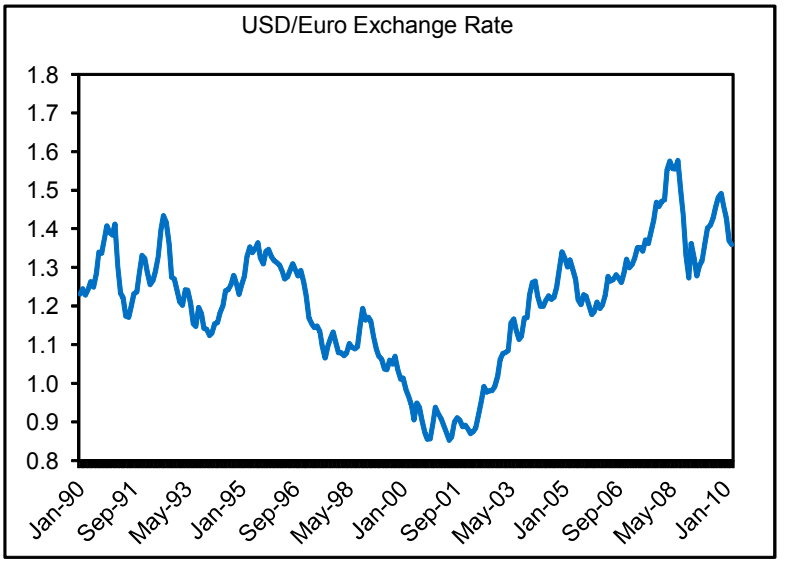

... but the real effective exchange rate remains close to its equilibrium.

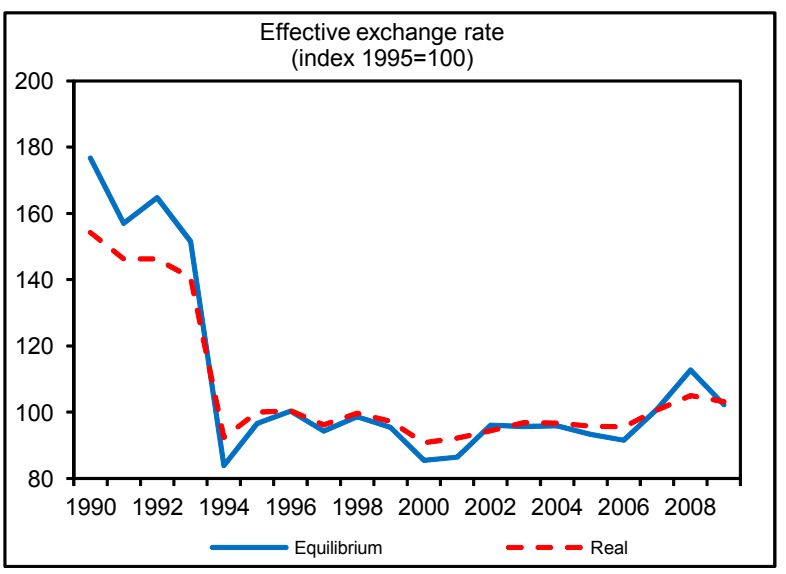

... as well as the business climate.

\begin{tabular}{|lcc|}
\hline \multicolumn{3}{|c|}{ Doing Bus iness Indicators } \\
\hline \multicolumn{2}{|c|}{2009} & 2010 \\
\hline Overall ranking & 152 & 157 \\
Starting a business & 94 & 102 \\
Dealing w ith construction permits & 121 & 124 \\
Employing w orkers & 173 & 172 \\
Registering property & 164 & 166 \\
Getting credit & 147 & 150 \\
Protecting investors & 164 & 165 \\
Paying taxes & 173 & 172 \\
Trading across borders & 64 & 57 \\
Enforcing contracts & 150 & 151 \\
Closing a business & 80 & 80 \\
Number of countries & 181 & 181 \\
\hline
\end{tabular}

Sources: IMF staff calculations and estimates; World Economic Forum; and World Bank. 


\section{Financial Sector Reform}

18. The global crisis has had negligible effects on the banking system, but the sector is affected by the slowdown in economic growth. Bank loan quality has deteriorated because of domestic economic conditions. As in other countries in the WAEMU region, compliance with prudential requirements has historically been mixed (Tables below). But most banks are on track to meet the higher minimum capital requirements by December 2010. The authorities are assessing the position of credit institutions likely to encounter difficulties in complying with the new rules and proposing targeted measures. Some private sector participants saw a need to review some prudential requirements (e.g., on the maturity matching of assets and resources), which may be too conservative and hinder market development.

\begin{tabular}{lrrrrrrrr}
\multicolumn{1}{c}{ Financial Soundness Indicators for the Banking Sector, 2002-09 } \\
(Percent)
\end{tabular}

$1 /$ Changes in 2006 due to ICS. It was recapitalized in 2008 but past provisions remain in place.

Number of Banks Non-Compliant with Prudential Standards

\begin{tabular}{|c|c|c|c|c|c|c|c|c|}
\hline & Dec-02 & Dec-03 & Dec-04 & Dec-05 & Dec-06 & Dec-07 & Dec-08 & Dec-09 \\
\hline Minimum capital $1 /$ & 2 & 1 & 1 & 1 & 1 & 1 & 0 & 2 \\
\hline Capital adequacy $2 /$ & 2 & 2 & 2 & 2 & 2 & 2 & 2 & 2 \\
\hline Large exposures and concentration 3/ & 2 & 6 & 6 & 8 & 5 & 6 & 8 & 6 \\
\hline Liquidity $4 /$ & 1 & 3 & 1 & 4 & 4 & 4 & 4 & 5 \\
\hline Transformation (stable resources) 5/ & 5 & 3 & 4 & 3 & 4 & 6 & 6 & 6 \\
\hline Number of Banks & 11 & 11 & 12 & 14 & 17 & 17 & 17 & 17 \\
\hline
\end{tabular}

Source: BCEAO and BC-WAMU

1/ Capital equity $>$ CFAF 1 billion.

2/ Regulatory capital/risk-weighted assets $>8$ percent.

3/ (i) Loans to a single borrower/regulatory capital < 75 percent; (ii) Sum of all risks reaching 25 percent of regulatory capital $<8$ times regulatory capital.

4/ Assets with residual term of less than 3 months/liabilities with residual term of less than 3 months $>75$ percent.

5 / Resources with residual term of more than 2 years/assets with residual term of more than two years $>75$ percent.

19. Stress tests by the authorities suggest the banking system may have some resilience to large credit shocks. Based on December 2009 data, if credit quality worsened and provisioning needed to increase by 50 percent, the global capital adequacy of the banking system would deteriorate by less than 1 percentage point, and no additional bank would violate the regulation on the minimum capital adequacy ratio. Even if nonperforming loans increased by 25 percent, the capital adequacy ratio of the banking system as a whole would be well above the 8 percent threshold at 14.5 percent, although a third bank would breach the regulation.

20. Authorities and staff saw scope to improve the contribution of the financial sector to investment and long-run growth (Figure 6). 
Figure 6. Senegal: Financial Sector Issues

Overall, the banking sector appears well capitalized..

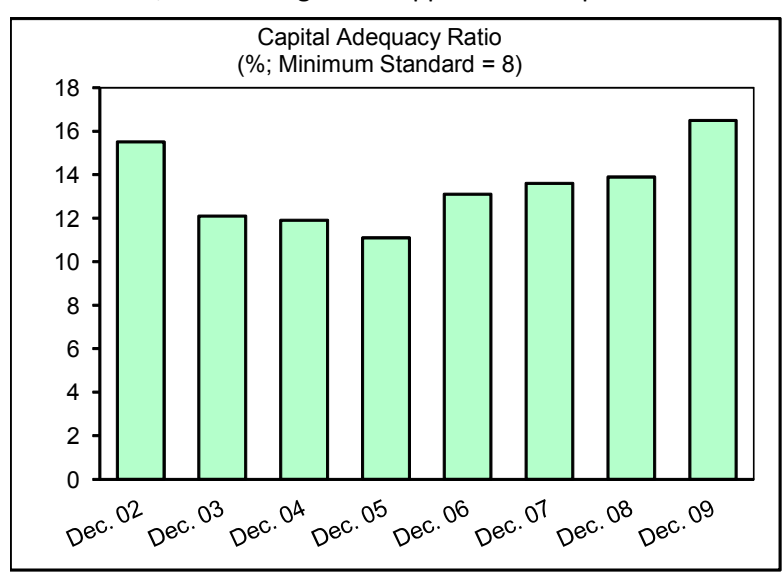

...non-performing loans are high,...

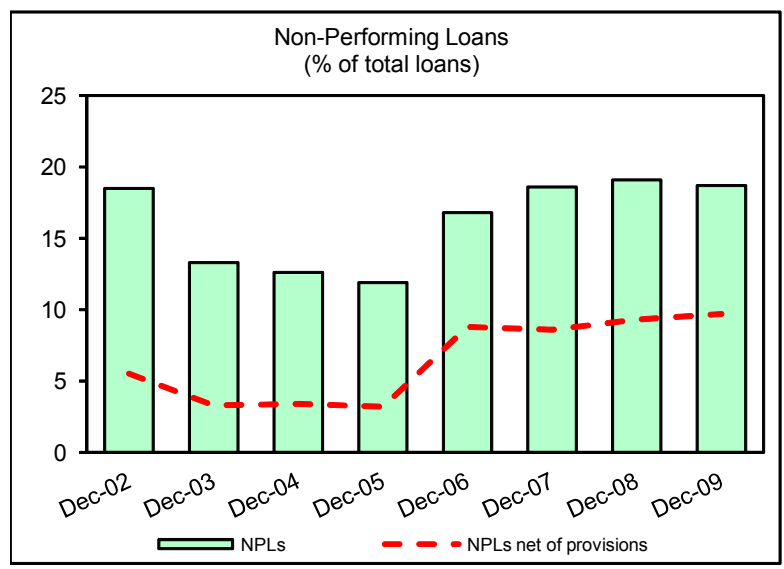

The gradual deepening of the banking market...

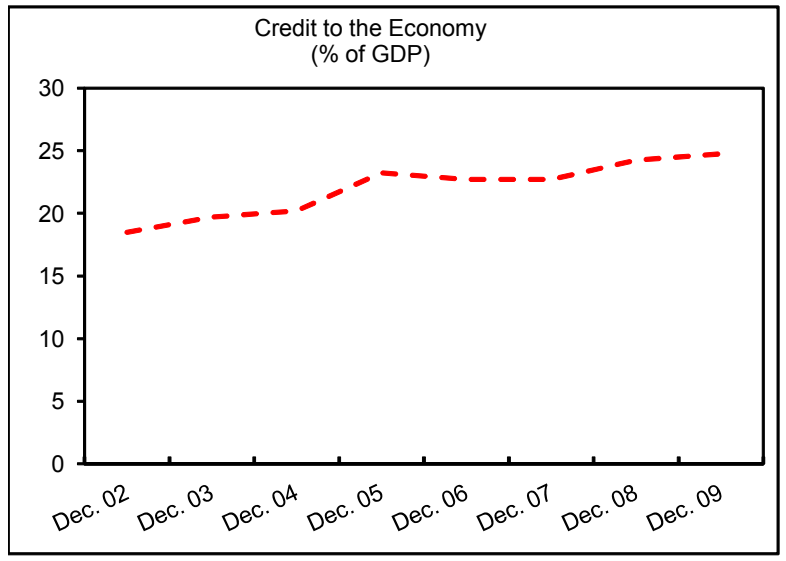

...but loan concentration is high,...

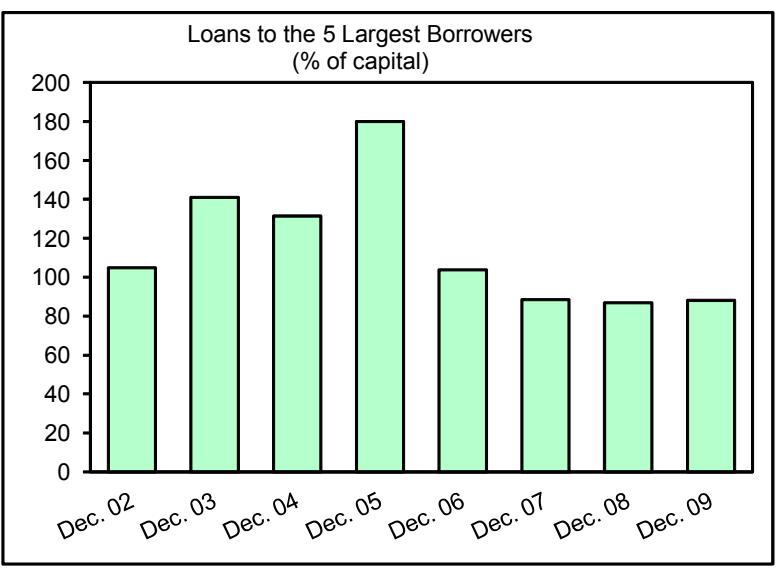

...and banks' asset maturity is relatively short.

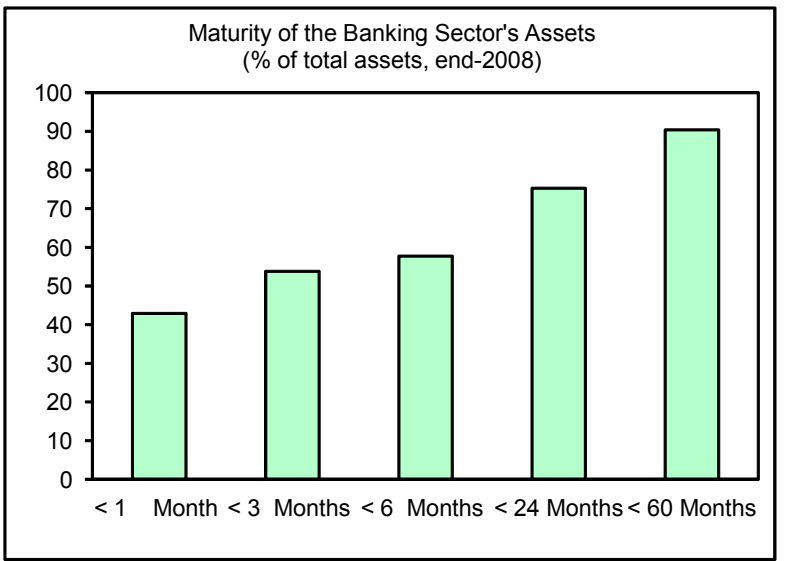

...would be supported by overcoming obstacles to credit access.

\begin{tabular}{|c|c|c|c|}
\hline \multicolumn{4}{|c|}{ Access to Credit $^{1}$} \\
\hline & Senegal & Mauritius & Morocco \\
\hline $\begin{array}{l}\text { Rank } \\
\text { (out of 183) }\end{array}$ & 150 & 87 & 87 \\
\hline $\begin{array}{l}\text { Strength of Legal Rights Index } \\
(0-10)\end{array}$ & 3 & 5 & 3 \\
\hline $\begin{array}{l}\text { Credit Information Index } \\
(0-6)\end{array}$ & 1 & 3 & 5 \\
\hline $\begin{array}{l}\text { Borrow ers Covered by Public Registery } \\
\text { (\%of adults) }\end{array}$ & 4 & 37 & 0 \\
\hline $\begin{array}{l}\text { Borrow ers Covered by Private Registery } \\
\text { (\%of adults) }\end{array}$ & 0 & 0 & 14 \\
\hline
\end{tabular}

Sources: Senegalese authorities; World Bank; and IMF staff calculations and estimates.

${ }^{1}$ Doing Business 2010. 
Surveys of firms identify inadequate access to finance as the leading constraint to private sector development in Senegal. ${ }^{6}$ The authorities have taken stock of various reform initiatives in an updated consolidated financial sector action plan (completed structural benchmark), taking into account progress since the 2004 national FSAP and the 2007 regional FSAP. The action plan lists a number of decisions to be taken in 2010-2011 to further improving the institutional, legal, and operational environment of the financial sector, including increasing the rate of bank penetration, while at the same time guarding against vulnerabilities.

\section{Program Issues}

21. The appended Letter of Intent and accompanying Memorandum of Economic and Financial Policies outlines the authorities' policy objectives for the remainder of 2010. Quantitative assessment criteria for end-June 2010 are shown in Table 1 and structural measures in Table 2 of Appendix I (see also Text Table 2). The thrust of the structural measures remains unchanged.

\section{The program includes an adjuster for the basic fiscal balance if additional} privatization revenues materialize. In that case, the floor on the basic fiscal balance will be adjusted downward by up to CFAF 35 billion. The adjuster would help the authorities to sustain their original spending plans. Any additional privatization receipts could support new infrastructure projects in next year's budget.

\section{The program faces risks, notwithstanding the authorities' commitment to their} program. These risks include continued revenue shortfalls, liquidity shortages in the regional market, delays in implementing domestic reforms, and institutional and policy changes in the run-up to elections intensifying spending pressures. Increased vigilance with the contracting of external loans is also needed, in particular because two Ministers have signature authority for external loans.

24. The future relationship between Senegal and the Fund was discussed. The PSI ends in early November. The authorities and staff expect continued close collaboration. Discussions on a future program will take place closer to that time (Box 4).

\footnotetext{
${ }^{6}$ World Economic Forum, Global Competitiveness Report 2009-2010.
} 
Text Table 2. Structural Benchmarks, May-October 2010

\begin{tabular}{|c|c|c|c|}
\hline Measures & $\begin{array}{c}\text { MEFP } \\
\S\end{array}$ & $\begin{array}{c}\text { Implementation } \\
\text { Date }\end{array}$ & $\begin{array}{c}\text { Macroeconomic } \\
\text { Rationale }\end{array}$ \\
\hline $\begin{array}{l}\text { 1. Submit a supplementary budget for } 2010 \\
\text { to Parliament, based on the macroeconomic } \\
\text { framework agreed with IMF staff, which } \\
\text { includes the specific line items authorizing } \\
\text { the Ministry of Finance to settle in } 2010 \text { the } \\
\text { extrabudgetary arrears of the ministries and } \\
\text { public institutions and agencies and part of } \\
\text { the public institution and agency debt } \\
\text { identified in the July } 2009 \text { audit and not yet } \\
\text { budgeted for. Any debt settlement not } \\
\text { requiring a budget allocation will be } \\
\text { presented in an annex to the supplementary } \\
\text { budget. This process is accompanied by a } \\
\text { press release explaining the settlement terms } \\
\text { and conditions. }\end{array}$ & 5,9 & May 15, 2010 & $\begin{array}{l}\text { Complete the } \\
\text { normalization of financial } \\
\text { relations with the private } \\
\text { sector. }\end{array}$ \\
\hline $\begin{array}{l}\text { 2. Improve SIGFIP by including payroll } \\
\text { expenditure and implementing the SIGFIP- } \\
\text { ASTER interface. }\end{array}$ & 18 & July 31,2010 & $\begin{array}{l}\text { Strengthen the } \\
\text { monitoring of budget } \\
\text { execution. }\end{array}$ \\
\hline $\begin{array}{l}\text { 3. Conduct a census of all accounts of } \\
\text { general government and public institutions, } \\
\text { including the number, name of the holder, } \\
\text { and the balances as of December } 31,2008 \\
\text { and } 2009 \text {, for each account, with a view to } \\
\text { adopting a strategy for establishing a single } \\
\text { Treasury account. }\end{array}$ & 20 & July 31,2010 & $\begin{array}{l}\text { Improve liquidity } \\
\text { management and the } \\
\text { transparency of } \\
\text { operations. }\end{array}$ \\
\hline $\begin{array}{l}\text { 4. Complete payment of at least } 50 \text { percent of } \\
\text { extrabudgetary arrears and public institution } \\
\text { and agency debt identified in the July } 2009 \\
\text { audit. }\end{array}$ & 9 & $\begin{array}{c}\text { September } 30, \\
2010\end{array}$ & $\begin{array}{l}\text { Complete normalization } \\
\text { of financial relations with } \\
\text { the private sector and } \\
\text { improve the business } \\
\text { climate. }\end{array}$ \\
\hline $\begin{array}{l}\text { 5. Publish a press release reporting on the } \\
\text { results of the internal audit, summarizing } \\
\text { progress made to clear the extrabudgetary } \\
\text { commitments and public institution and } \\
\text { agency debt, and detailing steps taken to } \\
\text { complete this process. }\end{array}$ & 10 & $\begin{array}{l}\text { September } 30 \\
2010\end{array}$ & $\begin{array}{l}\text { Complete normalization } \\
\text { of financial relations with } \\
\text { the private sector and } \\
\text { improve the business } \\
\text { climate. }\end{array}$ \\
\hline $\begin{array}{l}\text { 6. Compile the general tax code and all } \\
\text { legislation governing domestic taxation in a } \\
\text { single document. }\end{array}$ & 23 & October 15,2010 & $\begin{array}{l}\text { Further simplify the tax } \\
\text { system. }\end{array}$ \\
\hline
\end{tabular}




\section{Box 4. Key Medium-Term Policy Issues}

Key issues are to accelerate growth, reduce vulnerabilities to shocks, and protect the most vulnerable. The following reform areas will play a key role, and part of these could form cornerstones for a next program with the Fund:

- $\quad$ Continued PFM reforms: Significant progress has been made since late 2008, but further public financial management reforms remain essential for more effective expenditure planning and control.

- $\quad$ Raising tax revenues: While already high in a regional perspective, the authorities aim to increase tax revenues above 20 percent of GDP. Higher revenues, supported by further strengthening tax and customs administrations, rationalizing tax expenditure, and eventually other tax reform measures, would increase the fiscal space for priority spending.

- $\quad$ Expenditure composition and quality of spending: The authorities' emphasis on the importance of public investment, including infrastructure development, would be supported by improved investment planning and a well-developed debt management strategy.

- $\quad$ Private sector development: Further reforms to support private sector development, including in the business climate and governance, are essential for raising growth and central to the authorities' efforts. The energy and financial sectors will need to play a supportive role.

\section{Staff Appraisal}

25. There are signs that Senegal's economy has started to recover. Growth is projected to reach $3 \frac{1}{2}$ percent in 2010, driven by a pick-up in external demand and supportive domestic economic policies.

\section{While the risks of debt distress are low, the authorities will need to gradually} reduce the fiscal stimulus to reach a deficit target of 4 percent of GDP in the medium term and somewhat less in the longer run, which is consistent with debt sustainability.

\section{A swift and transparent settlement of extrabudgetary spending and agency debt is} essential. Fast settlement will support the recovery and the authorities' fiscal objectives of raising growth-enhancing public investments and sheltering priority spending.

28. Raising Senegal's growth potential requires faster action on the authorities' plans, which specify improvements in nonprice competitiveness through a better business climate and governance. In the energy sector, which is critical for growth, the plan, supported by the Bank and other donors, needs to be implemented quickly to limit supply bottlenecks and fiscal risks. 
29. The contribution of the financial sector to growth needs to be enhanced. As the financial sector action plan makes clear, it is essential to improve the institutional, legal, and operational environment while at the same time guarding against vulnerabilities. The space for greater financing by the financial sector of the government should be examined.

30. While program implementation on the macroeconomic side has been mixed, solid progress has been made on the structural side. The staff recommends that the waiver for the nonobservance of the performance/assessment criterion on the fiscal balance be granted and the fifth review of the PSI and the third and last review of the ESF arrangement be completed. The 2010 budget includes a contingency reserve, and the authorities are committed to making every effort in the future to offset any increase in expenditure related to supplemental appropriation decrees (décrets d'avance) by reducing nonpriority spending.

31. It is recommended that the next Article IV consultation take place within 24 months, subject to the provisions of the decision on consultation cycles in program countries. 
Table 1. Senegal: Selected Economic and Financial Indicators, 2007-15

\begin{tabular}{|c|c|c|c|c|c|c|c|c|c|c|}
\hline & \multirow[t]{2}{*}{2007} & \multirow[t]{2}{*}{2008} & \multirow{2}{*}{$\begin{array}{r}2009 \\
\text { Est. }\end{array}$} & \multicolumn{2}{|c|}{2010} & 2011 & 2012 & 2013 & 2014 & 2015 \\
\hline & & & & Prog. & Proj. & \multicolumn{5}{|c|}{ Proj. } \\
\hline & \multicolumn{9}{|c|}{ (Annual percentage change) } & \\
\hline National income and prices & & & & & & & & & & \\
\hline GDP at constant prices & 4.8 & 2.3 & 1.5 & 3.4 & 3.4 & 4.1 & 4.5 & 4.7 & 4.9 & 5.0 \\
\hline Of which: nonagriculture GDP & 6.3 & 1.1 & 1.1 & 3.4 & 3.4 & 4.1 & 4.6 & 4.8 & 5.0 & 5.1 \\
\hline GDP deflator & 5.4 & 7.3 & -0.5 & 2.1 & 2.1 & 2.1 & 2.1 & 2.1 & 2.1 & 2.1 \\
\hline \multicolumn{11}{|l|}{ Consumer prices } \\
\hline Annual average & 5.9 & 5.8 & -1.1 & 1.8 & 1.6 & 2.1 & 2.1 & 2.1 & 2.1 & 2.1 \\
\hline End of period & 6.2 & 4.3 & -2.2 & 2.2 & 2.1 & 2.1 & 2.1 & 2.1 & 2.1 & 2.1 \\
\hline \multicolumn{11}{|l|}{ External sector } \\
\hline Exports, f.o.b. (CFA francs) & -3.7 & 23.0 & -15.2 & 14.6 & 17.6 & 9.3 & 7.1 & 8.4 & 7.5 & 7.5 \\
\hline Imports, f.o.b. (CFA francs) & 19.5 & 25.8 & -20.4 & 10.8 & 10.3 & 6.7 & 7.1 & 7.9 & 7.9 & 6.7 \\
\hline Export volume & 6.1 & -14.6 & 9.9 & 10.5 & 5.2 & 5.9 & 6.0 & 6.6 & 6.2 & 5.9 \\
\hline Import volume & 21.5 & 11.3 & -5.3 & 5.1 & 4.6 & 4.9 & 6.7 & 6.4 & 6.5 & 6.2 \\
\hline Terms of trade ("-" = deterioration) & -2.9 & 12.2 & -1.0 & -1.6 & 4.8 & 1.7 & 0.9 & 0.7 & 0.1 & 0.7 \\
\hline Nominal effective exchange rate & 1.9 & 2.9 & -0.2 & $\ldots$ & $\ldots$ & $\ldots$ & $\ldots$ & $\ldots$ & $\ldots$ & $\ldots$ \\
\hline Real effective exchange rate & 5.3 & 4.4 & -1.7 & $\ldots$ & $\ldots$ & $\ldots$ & $\ldots$ & $\ldots$ & $\ldots$ & $\ldots$ \\
\hline \multicolumn{11}{|c|}{ (Changes in percent of beginning-of-year broad money, unless otherwise indicated) } \\
\hline \multicolumn{11}{|l|}{ Money and credit } \\
\hline Net domestic assets & 8.6 & 6.2 & 6.1 & 13.0 & 10.1 & 10.0 & 9.0 & 9.2 & 10.5 & 7.3 \\
\hline Domestic credit & 11.5 & 7.3 & 6.8 & 13.4 & 10.0 & 10.3 & 9.2 & 9.4 & 10.7 & 7.6 \\
\hline Credit to the government (net) & 4.9 & -3.5 & 4.2 & 8.3 & 4.7 & 4.2 & 3.0 & 2.8 & 3.9 & 0.8 \\
\hline \multirow[t]{2}{*}{ Credit to the economy (percentage growth) } & 10.5 & 17.2 & 3.6 & 7.5 & 7.8 & 9.4 & 9.7 & 10.4 & 11.0 & 11.1 \\
\hline & \multicolumn{9}{|c|}{ (Percent of GDP, unless otherwise indicated) } & \\
\hline \multicolumn{11}{|l|}{ Government financial operations } \\
\hline Revenue & 21.1 & 19.4 & 18.7 & 19.6 & 19.4 & 19.6 & 19.9 & 20.1 & 20.4 & 20.6 \\
\hline Grants & 2.6 & 2.4 & 3.0 & 2.3 & 2.4 & 2.2 & 2.2 & 2.2 & 2.2 & 2.2 \\
\hline Total expenditure and net lending & 27.6 & 26.6 & 27.1 & 26.9 & 26.3 & 26.1 & 26.1 & 26.3 & 26.6 & 26.7 \\
\hline \multicolumn{11}{|l|}{ Overall fiscal surplus $(+)$ or deficit (-) } \\
\hline Payment order basis, excluding grants & -6.2 & -7.0 & -8.1 & -7.3 & -6.9 & -6.5 & -6.2 & -6.2 & -6.2 & -6.2 \\
\hline Payment order basis, including grants & -3.7 & -4.6 & -5.1 & -4.9 & -4.5 & -4.3 & -4.0 & -4.0 & -4.0 & -4.0 \\
\hline Primary fiscal balance $1 /$ & -3.3 & -4.2 & -4.7 & -4.1 & -4.1 & -3.4 & -3.0 & -3.0 & -3.0 & -2.9 \\
\hline Basic fiscal balance $2 /$ & -1.0 & -0.8 & -2.8 & -1.9 & -1.9 & -1.1 & -0.8 & -0.8 & -0.7 & -0.7 \\
\hline Gross domestic investment & 34.5 & 33.8 & 28.5 & 30.9 & 29.1 & 29.8 & 30.2 & 31.0 & 31.7 & 32.3 \\
\hline Government & 11.2 & 10.0 & 10.5 & 11.2 & 10.7 & 10.6 & 10.7 & 10.9 & 11.2 & 11.3 \\
\hline Nongovernment & 23.3 & 23.8 & 18.1 & 19.6 & 18.5 & 19.2 & 19.5 & 20.1 & 20.5 & 21.0 \\
\hline Gross domestic savings & 12.1 & 7.3 & 8.5 & 11.8 & 9.6 & 10.6 & 11.3 & 12.4 & 12.8 & 13.7 \\
\hline Government & 7.1 & 5.0 & 5.4 & 7.3 & 7.2 & 7.4 & 7.8 & 8.1 & 8.3 & 8.5 \\
\hline Nongovernment & 4.9 & 2.3 & 3.1 & 4.5 & 2.5 & 3.2 & 3.4 & 4.3 & 4.5 & 5.1 \\
\hline Gross national savings & 22.7 & 19.5 & 19.8 & 21.0 & 20.4 & 20.9 & 21.1 & 21.8 & 22.3 & 23.1 \\
\hline \multicolumn{11}{|l|}{ External current account deficit (-) } \\
\hline Including current official transfers & -11.8 & -14.3 & -8.7 & -9.9 & -8.7 & -9.0 & -9.1 & -9.2 & -9.4 & -9.2 \\
\hline Excluding current official transfers & -13.2 & -15.3 & -9.8 & -10.8 & -9.8 & -9.9 & -10.0 & -10.2 & -10.3 & -10.1 \\
\hline Central government domestic debt $3 /$ & 6.6 & 5.3 & 8.1 & 7.0 & 8.6 & 10.2 & 11.1 & 11.9 & 13.3 & 13.0 \\
\hline External public debt (nominal) 3/ 4/ & 17.9 & 19.8 & 24.0 & 26.1 & 27.1 & 27.8 & 28.5 & 29.1 & 28.9 & 29.6 \\
\hline \multicolumn{11}{|l|}{ External public debt service $4 /$} \\
\hline Percent of exports & 3.4 & 2.8 & 3.8 & 4.2 & 4.8 & 6.6 & 6.3 & 6.0 & 9.4 & 5.2 \\
\hline Percent of government revenue & 4.2 & 3.8 & 4.8 & 4.8 & 6.2 & 8.5 & 8.0 & 7.6 & 11.8 & 6.5 \\
\hline Gross domestic product (CFAF billions) & 5,405 & 5,935 & 5,999 & 6,336 & 6,345 & 6,742 & 7,197 & 7,696 & 8,241 & 8,836 \\
\hline
\end{tabular}

Sources: Senegalese authorities; and IMF staff estimates and projections.

$1 /$ Defined as total revenue and grants minus total expenditure and net lending, excluding interest expenditure.

2/ Defined as total revenue minus total expenditure and net lending, excluding externally financed capital expenditure, on-lending,

HIPC and MDRI spending, and 2010 clearing of extrabudgetary spending and agency debt.

3/ Debt outstanding at year-end.

4/ After HIPC and MDRI (from 2006) debt relief. 
Table 2. Senegal: Balance of Payments, 2007-15

\begin{tabular}{|c|c|c|c|c|c|c|c|c|c|}
\hline & 2007 & 2008 & 2009 & 2010 & 2011 & 2012 & 2013 & 2014 & 2015 \\
\hline & & & Est. & \multicolumn{6}{|c|}{ Proj. } \\
\hline & \multicolumn{9}{|c|}{ (CFAF billions, unless otherwise indicated) } \\
\hline Current account & -638 & -850 & -524 & -555 & -604 & -655 & -710 & -774 & -809 \\
\hline Balance on goods & $-1,193$ & $-1,523$ & $-1,159$ & $-1,217$ & $-1,274$ & $-1,365$ & $-1,468$ & $-1,589$ & $-1,686$ \\
\hline Exports, f.o.b. & 803 & 988 & 838 & 985 & 1,076 & 1,153 & 1,250 & 1,343 & 1,443 \\
\hline Imports, f.o.b. & $-1,996$ & $-2,510$ & $-1,997$ & $-2,202$ & $-2,350$ & $-2,518$ & $-2,717$ & $-2,931$ & $-3,129$ \\
\hline Services and incomes (net) & -64 & -82 & -64 & -56 & -62 & -43 & -16 & -15 & -17 \\
\hline Credits & 671 & 709 & 684 & 715 & 737 & 774 & 822 & 881 & 944 \\
\hline Debits & -734 & -791 & -748 & -771 & -799 & -817 & -838 & -896 & -961 \\
\hline Of which: interest on public debt & -24 & -24 & -23 & -29 & -29 & -29 & -29 & -29 & -32 \\
\hline Unrequited current transfers (net) & 618 & 754 & 699 & 718 & 732 & 753 & 773 & 830 & 892 \\
\hline Private (net) $1 /$ & 566 & 722 & 659 & 677 & 698 & 713 & 729 & 780 & 837 \\
\hline Public (net) & 52 & 33 & 40 & 41 & 34 & 39 & 45 & 50 & 55 \\
\hline Of which: budgetary grants & 53 & 38 & 46 & 45 & 36 & 38 & 41 & 44 & 47 \\
\hline Capital and financial account & 690 & 745 & 707 & 523 & 622 & 688 & 768 & 852 & 915 \\
\hline Capital account & 182 & 111 & 144 & 115 & 172 & 180 & 189 & 199 & 209 \\
\hline Private capital transfers & 7 & 8 & 8 & 8 & 9 & 9 & 9 & 9 & 10 \\
\hline Project grants & 86 & 101 & 136 & 107 & 114 & 121 & 130 & 139 & 149 \\
\hline Debt cancellation and other transfers $2 /$ & 89 & 2 & 0 & 0 & 50 & 50 & 50 & 50 & 50 \\
\hline Financial account & 508 & 634 & 563 & 407 & 449 & 507 & 579 & 654 & 706 \\
\hline Direct investment & 131 & 122 & 98 & 112 & 127 & 162 & 192 & 261 & 272 \\
\hline Portfolio investment & 29 & 21 & 27 & 38 & 63 & 53 & 51 & 71 & 39 \\
\hline Other investment & 348 & 491 & 438 & 257 & 259 & 293 & 336 & 322 & 395 \\
\hline Public sector (net) & 97 & 208 & 341 & 154 & 143 & 158 & 176 & 136 & 244 \\
\hline Of which: disbursements & 158 & 264 & 363 & 205 & 223 & 240 & 260 & 288 & 307 \\
\hline program loans & 19 & 70 & 55 & 29 & 32 & 34 & 37 & 39 & 42 \\
\hline project loans & 138 & 192 & 128 & 176 & 191 & 206 & 223 & 249 & 265 \\
\hline other & 2 & 2 & 180 & 0 & 0 & 0 & 0 & 0 & 0 \\
\hline Of which: SDR allocation & 0 & 0 & 93 & 0 & 0 & 0 & 0 & 0 & 0 \\
\hline amortization & -54 & -44 & -50 & -51 & -80 & -83 & -84 & -152 & -63 \\
\hline Private sector (net) & 254 & 279 & 98 & 102 & 116 & 135 & 160 & 186 & 152 \\
\hline Errors and omissions & -4 & 5 & 0 & 0 & 0 & 0 & 0 & 0 & 0 \\
\hline Overall balance & 51 & -105 & 183 & -32 & 17 & 33 & 58 & 79 & 107 \\
\hline Financing & -51 & 105 & -183 & 9 & -17 & -33 & -58 & -79 & -107 \\
\hline Net foreign assets (BCEAO) & -75 & -8 & -179 & -3 & -27 & -43 & -68 & -88 & -116 \\
\hline Net use of Fund resources & 0 & 17 & 47 & 0 & -2 & -3 & -3 & -9 & -18 \\
\hline Purchases/disbursements & 0 & 17 & 47 & 0 & 0 & 0 & 0 & 0 & 0 \\
\hline Repurchases/repayments & 0 & 0 & 0 & 0 & -2 & -3 & -3 & -9 & -18 \\
\hline Other & -75 & -25 & -226 & -2 & -26 & -41 & -65 & -80 & -98 \\
\hline Deposit money banks & 3 & 98 & -24 & -7 & -7 & -7 & -8 & -8 & -9 \\
\hline Payments arrears ("-" = reduction) & 0 & 0 & 0 & 0 & 0 & 0 & 0 & 0 & 0 \\
\hline Exceptional financing & 21 & 16 & 20 & 18 & 17 & 18 & 18 & 18 & 18 \\
\hline Residual financing gap $3 /$ & 0 & 0 & 0 & 24 & 0 & 0 & 0 & 0 & 0 \\
\hline \multicolumn{10}{|l|}{ Memorandum items: } \\
\hline \multicolumn{10}{|l|}{ Current account balance } \\
\hline Including current official transfers (percent of GDP) & -11.8 & -14.3 & -8.7 & -8.7 & -9.0 & -9.1 & -9.2 & -9.4 & -9.2 \\
\hline Excluding current official transfers (percent of GDP) & -13.2 & -15.3 & -9.8 & -9.8 & -9.9 & -10.0 & -10.2 & -10.3 & -10.1 \\
\hline Gross official reserves (imputed reserves, billions of US $\$$ ) & 1.6 & 1.5 & 2.1 & 2.0 & 2.1 & 2.1 & 2.3 & 2.4 & 2.6 \\
\hline (percent of broad money) & 37.2 & 37.1 & 43.4 & 39.8 & 36.8 & 34.6 & 33.0 & 31.3 & 30.7 \\
\hline WAEMU gross official reserves (billions of US\$) & 10.7 & 10.5 & 13.8 & $\ldots$ & $\ldots$ & $\ldots$ & $\ldots$ & $\ldots$ & $\ldots$ \\
\hline (percent of broad money) & 56.6 & 55.0 & 58.7 & $\ldots$ & $\ldots$ & $\ldots$ & $\ldots$ & $\ldots$ & $\ldots$ \\
\hline (months of WAEMU imports of GNFS) & 6.2 & 5.7 & 7.4 & & & & $\ldots$ & $\ldots$ & \\
\hline Gross domestic product & 5,405 & 5,935 & 5,999 & 6,345 & 6,742 & 7,197 & 7,696 & 8,241 & 8,836 \\
\hline
\end{tabular}

Sources: Central Bank of West African States (BCEAO); and IMF staff estimates and projections.

1/ Upwardly revised from 2008 based on a new survey of workers' remittances.

2/ Includes receipts from sale of a telecom license in 2007 and MCA grants during 2011-15.

3/ Financing gap in 2010 to be filled with ESF drawing 
Table 3. Senegal: Government Financial Operations, 2007-15

\begin{tabular}{|c|c|c|c|c|c|c|c|c|c|c|c|}
\hline & \multirow[t]{2}{*}{2007} & \multirow[t]{2}{*}{2008} & \multicolumn{2}{|c|}{2009} & \multicolumn{2}{|c|}{2010} & \multirow[t]{2}{*}{2011} & \multirow[t]{2}{*}{2012} & \multirow{2}{*}{$\begin{array}{l}2013 \\
\text { Proj. }\end{array}$} & \multirow[t]{2}{*}{2014} & \multirow[t]{2}{*}{2015} \\
\hline & & & Prog. & Est. & Prog. & Proj. & & & & & \\
\hline & \multicolumn{11}{|c|}{ (Billions of CFA francs, unless otherwise indicated) } \\
\hline Total revenue and grants & 1,277 & 1,293 & 1,323 & 1,303 & 1,390 & 1,380 & 1,472 & 1,591 & 1,719 & 1,861 & 2,012 \\
\hline Revenue & 1,139 & 1,153 & 1,160 & 1,121 & 1,242 & 1,228 & 1,322 & 1,431 & 1,548 & 1,678 & 1,816 \\
\hline Tax revenue & 1,088 & 1,088 & 1,125 & 1,084 & 1,199 & 1,170 & 1,260 & 1,365 & 1,477 & 1,603 & 1,735 \\
\hline Income tax & 232 & 273 & 284 & 287 & 300 & 303 & 328 & 357 & 389 & 424 & 463 \\
\hline Taxes on goods and services & 628 & 616 & 625 & 615 & 663 & 660 & 709 & 767 & 832 & 902 & 979 \\
\hline Taxes on petroleum products & 215 & 199 & 216 & 182 & 235 & 207 & 223 & 241 & 256 & 276 & 293 \\
\hline Nontax revenue & 51 & 65 & 36 & 37 & 43 & 58 & 62 & 66 & 71 & 76 & 81 \\
\hline Grants & 138 & 140 & 162 & 182 & 148 & 152 & 150 & 160 & 171 & 183 & 196 \\
\hline Budgetary & 53 & 38 & 61 & 46 & 41 & 45 & 36 & 38 & 41 & 44 & 47 \\
\hline Budgeted development projects & 86 & 101 & 101 & 136 & 107 & 107 & 114 & 121 & 130 & 139 & 149 \\
\hline Total expenditure and net lending & 1,491 & 1,579 & 1,592 & 1,623 & 1,703 & 1,668 & 1,760 & 1,877 & 2,026 & 2,190 & 2,363 \\
\hline Current expenditure & 881 & 979 & 957 & 993 & 989 & 989 & 1,043 & 1,108 & 1,185 & 1,270 & 1,367 \\
\hline Wages and salaries $1 /$ & 327 & 348 & 370 & 364 & 397 & 397 & 411 & 432 & 462 & 494 & 530 \\
\hline Interest due & 34 & 39 & 48 & 41 & 53 & 53 & 62 & 70 & 77 & 84 & 97 \\
\hline Of which : external 2/ & 24 & 24 & 26 & 23 & 31 & 29 & 29 & 29 & 29 & 29 & 32 \\
\hline Other current expenditure & 519 & 593 & 539 & 587 & 539 & 539 & 570 & 606 & 647 & 691 & 740 \\
\hline Transfers and subsidies $3 /$ & 287 & 333 & 270 & 286 & 250 & 250 & 266 & 284 & 303 & 325 & 348 \\
\hline Of which: SAR and butane subsidy & 55 & 69 & 44 & 33 & 0 & 0 & 0 & 0 & 0 & 0 & 0 \\
\hline Of which: SENELEC & 0 & 30 & 18 & 30 & 0 & 0 & 0 & 0 & 0 & 0 & 0 \\
\hline Of which: Food subsidies & 21 & 46 & 0 & 0 & 0 & 0 & 0 & 0 & 0 & 0 & 0 \\
\hline Goods and services & 217 & 239 & 256 & 292 & 277 & 277 & 293 & 311 & 332 & 355 & 380 \\
\hline HIPC and MDRI current spending & 15 & 21 & 13 & 9 & 12 & 12 & 12 & 12 & 11 & 11 & 12 \\
\hline Capital expenditure 4/ & 605 & 595 & 633 & 627 & 713 & 678 & 717 & 769 & 841 & 920 & 996 \\
\hline Domestically financed & 392 & 314 & 386 & 369 & 439 & 404 & 423 & 452 & 494 & 539 & 587 \\
\hline HIPC and MDRl-financed & 60 & 63 & 42 & 60 & 48 & 48 & 48 & 46 & 46 & 47 & 48 \\
\hline Non-HIPC/MDRI financed & 331 & 251 & 344 & 309 & 392 & 357 & 375 & 406 & 448 & 492 & 539 \\
\hline Externally financed & 213 & 281 & 247 & 258 & 273 & 273 & 294 & 316 & 347 & 381 & 409 \\
\hline Net lending & 5 & 5 & 2 & 3 & 2 & 2 & 0 & 0 & 0 & 0 & 0 \\
\hline Of which: On-lending & 10 & 12 & 10 & 6 & 10 & 10 & 11 & 11 & 12 & 13 & 14 \\
\hline Selected public sector entities balance $5 /$ & 16 & 13 & 0 & 0 & 0 & 0 & 0 & 0 & 0 & 0 & 0 \\
\hline Primary fiscal balance & -163 & -235 & -221 & -263 & -260 & -235 & -227 & -216 & -231 & -244 & -254 \\
\hline Overall fiscal balance (including grants) & -198 & -273 & -269 & -304 & -313 & -288 & -289 & -286 & -307 & -328 & -351 \\
\hline Overall fiscal balance (excluding grants) & -336 & -413 & -431 & -486 & -462 & -440 & -438 & -446 & -478 & -511 & -547 \\
\hline Basic fiscal balance 6/ & -54 & -50 & -119 & -168 & -119 & -97 & -74 & -61 & -62 & -59 & -64 \\
\hline Financing & 198 & 273 & 269 & 304 & 313 & 288 & 289 & 286 & 307 & 328 & 351 \\
\hline External financing & 131 & 224 & 292 & 245 & 191 & 192 & 202 & 209 & 227 & 203 & 279 \\
\hline Drawings & 156 & 262 & 218 & 183 & 206 & 205 & 223 & 240 & 260 & 288 & 307 \\
\hline Program loans & 19 & 70 & 63 & 55 & 30 & 29 & 32 & 34 & 37 & 39 & 42 \\
\hline Project loans & 138 & 192 & 155 & 128 & 176 & 176 & 191 & 206 & 223 & 249 & 265 \\
\hline Amortization due & -54 & -44 & -50 & -50 & -52 & -51 & -80 & -83 & -84 & -152 & -63 \\
\hline Debt relief and HIPC Initiative assistance & 21 & 16 & 20 & 20 & 18 & 18 & 17 & 18 & 18 & 18 & 18 \\
\hline T-bills and bonds issued in WAEMU & 8 & -9 & 14 & 4 & 20 & 20 & 43 & 34 & 33 & 49 & 18 \\
\hline Nonconcessional loans for infrastructure developme & 0 & 0 & 90 & 87 & 0 & 0 & 0 & 0 & 0 & 0 & \\
\hline Domestic financing & 58 & 124 & 19 & 151 & 182 & 103 & 97 & 77 & 81 & 125 & 23 \\
\hline Banking system $7 /$ & 98 & -46 & 36 & 110 & 182 & 100 & 97 & 77 & 81 & 126 & 24 \\
\hline Of which: T-bills and bonds & 136 & -14 & -26 & 52 & 69 & 59 & 128 & 103 & 100 & 148 & 53 \\
\hline Nonbank financing & -40 & 169 & -17 & 41 & -1 & 3 & 0 & 0 & 0 & 0 & 0 \\
\hline Settlement of payment delays $8 /$ & 0 & -84 & -66 & -95 & -83 & -30 & -11 & 0 & 0 & 0 & 0 \\
\hline Errors and omissions & 9 & 9 & 0 & 3 & 0 & 0 & 0 & 0 & 0 & 0 & 0 \\
\hline Financing gap 9/ & 0 & 0 & 24 & 0 & 24 & 24 & 0 & 0 & 0 & 0 & 0 \\
\hline \multicolumn{12}{|l|}{ Memorandum items: } \\
\hline Budgetary float (program definition) & 55 & 66 & 45 & 45 & 45 & 45 & 45 & 45 & 45 & 45 & 0 \\
\hline New issues of government securities & 183 & 131 & 127 & 147 & 177 & 220 & $\ldots$ & $\ldots$ & $\ldots$ & $\ldots$ & $\ldots$ \\
\hline Priority expenditure (percent of total expenditure) 10/ & 32 & 33 & 34 & 34 & 35 & 35 & $\ldots$ & $\ldots$ & $\ldots$ & $\ldots$ & \\
\hline Gross domestic product & 5,405 & 5,935 & 5,983 & 5,999 & 6,336 & 6,345 & 6,742 & 7,197 & 7,696 & 8,241 & 8,836 \\
\hline
\end{tabular}

Sources: Senegalese authorities; and IMF staff estimates and projections.

1/ Excludes project-related wages and salaries, which are included in capital spending, and the salaries of autonomous agencies and health and education contractual workers, which are included in transfers and subsidies.

2/ From 2006 on, reflects post-MDRI debt service schedule.

3/ Excludes subsidies aimed at sector development policies, which are included in capital spending

4/ Includes recapitalization of SENELEC. The government provided CFAF 65 billion in 2007 under domestically-financed capital

expenditure, while budget support by the World Bank and France in 2008-10 specifically earmarked for the recapitalization is being provided under externally-financed capital expenditure.

$5 /$ Local governments, autonomous public sector entities (e.g., hospitals, universities), and the civil servants pension fund (FNR). 6/ Total revenue minus total expenditure and net lending, excluding externally financed capital expenditure, on-lending, HIPC/MDRI expenditure, and 2010 clearing of extrabudgetary spending and agency debt.

$7 /$ Includes the 10 -year CFAF loan from the BCEAO in 2009 equal to the general SDR allocation.

8/ Within the expenditure chain in 2008-09, and extrabudgetary spending and agency debt in 2009-11.

9/ Financing gap in 2010 to be filled with ESF drawing.

10/ Defined as expenditures on health, education, environment, the judiciary, social development, sewage, and rural irrigation. 
Table 4. Senegal: Government Financial Operations, 2007-15

\begin{tabular}{|c|c|c|c|c|c|c|c|c|c|c|c|}
\hline & \multirow[t]{2}{*}{2007} & \multirow[t]{2}{*}{2008} & \multicolumn{2}{|c|}{2009} & \multicolumn{2}{|c|}{2010} & \multirow[t]{2}{*}{2011} & \multirow[t]{2}{*}{2012} & \multirow{2}{*}{$\begin{array}{l}2013 \\
\text { Proj. }\end{array}$} & \multirow[t]{2}{*}{2014} & \multirow[t]{2}{*}{2015} \\
\hline & & & Prog. & Est. & Prog. & Proj. & & & & & \\
\hline & \multicolumn{11}{|c|}{ (Percent of GDP) } \\
\hline Total revenue and grants & 23.6 & 21.8 & 22.1 & 21.7 & 21.9 & 21.8 & 21.8 & 22.1 & 22.3 & 22.6 & 22.8 \\
\hline Revenue & 21.1 & 19.4 & 19.4 & 18.7 & 19.6 & 19.4 & 19.6 & 19.9 & 20.1 & 20.4 & 20.6 \\
\hline Tax revenue & 20.1 & 18.3 & 18.8 & 18.1 & 18.9 & 18.4 & 18.7 & 19.0 & 19.2 & 19.4 & 19.6 \\
\hline Income tax & 4.3 & 4.6 & 4.7 & 4.8 & 4.7 & 4.8 & 4.9 & 5.0 & 5.1 & 5.1 & 5.2 \\
\hline Taxes on goods and services & 11.6 & 10.4 & 10.4 & 10.3 & 10.5 & 10.4 & 10.5 & 10.7 & 10.8 & 11.0 & 11.1 \\
\hline Taxes on petroleum products & 4.0 & 3.4 & 3.6 & 3.0 & 3.7 & 3.3 & 3.3 & 3.3 & 3.3 & 3.4 & 3.3 \\
\hline Nontax revenue & 0.9 & 1.1 & 0.6 & 0.6 & 0.7 & 0.9 & 0.9 & 0.9 & 0.9 & 0.9 & 0.9 \\
\hline Grants & 2.6 & 2.4 & 2.7 & 3.0 & 2.3 & 2.4 & 2.2 & 2.2 & 2.2 & 2.2 & 2.2 \\
\hline Total expenditure and net lending & 27.6 & 26.6 & 26.6 & 27.1 & 26.9 & 26.3 & 26.1 & 26.1 & 26.3 & 26.6 & 26.7 \\
\hline Current expenditure & 16.3 & 16.5 & 16.0 & 16.5 & 15.6 & 15.6 & 15.5 & 15.4 & 15.4 & 15.4 & 15.5 \\
\hline Wages and salaries & 6.1 & 5.9 & 6.2 & 6.1 & 6.3 & 6.3 & 6.1 & 6.0 & 6.0 & 6.0 & 6.0 \\
\hline Interest payments $1 /$ & 0.6 & 0.6 & 0.8 & 0.7 & 0.8 & 0.8 & 0.9 & 1.0 & 1.0 & 1.0 & 1.1 \\
\hline Other current expenditure & 9.6 & 10.0 & 9.0 & 9.8 & 8.5 & 8.5 & 8.5 & 8.4 & 8.4 & 8.4 & 8.4 \\
\hline Of which: Goods and services & 4.0 & 4.0 & 4.3 & 4.9 & 4.4 & 4.4 & 4.3 & 4.3 & 4.3 & 4.3 & 4.3 \\
\hline Of which: Transfers and subsidies & 5.3 & 5.6 & 4.5 & 4.8 & 3.9 & 3.9 & 3.9 & 3.9 & 3.9 & 3.9 & 3.9 \\
\hline Of which: Energy and food subsidies & 1.4 & 2.4 & 1.0 & 1.0 & 0.0 & 0.0 & 0.0 & 0.0 & 0.0 & 0.0 & 0.0 \\
\hline HIPC and MDRI current spending & 0.3 & 0.3 & 0.2 & 0.2 & 0.2 & 0.2 & 0.2 & 0.2 & 0.1 & 0.1 & $\ldots$ \\
\hline Capital expenditure $2 /$ & 11.2 & 10.0 & 10.6 & 10.5 & 11.2 & 10.7 & 10.6 & 10.7 & 10.9 & 11.2 & 11.3 \\
\hline Domestically financed & 7.2 & 5.3 & 6.5 & 6.2 & 6.9 & 6.4 & 6.3 & 6.3 & 6.4 & 6.5 & 6.6 \\
\hline Of which: Without transfers to PEs & 6.0 & 4.7 & 6.5 & 6.2 & 6.9 & 6.4 & 6.3 & 6.3 & 6.4 & 6.5 & 6.6 \\
\hline Externally financed & 3.9 & 4.7 & 4.1 & 4.3 & 4.3 & 4.3 & 4.4 & 4.4 & 4.5 & 4.6 & 4.6 \\
\hline Net lending & 0.1 & 0.1 & 0.0 & 0.1 & 0.0 & 0.0 & 0.0 & 0.0 & 0.0 & 0.0 & 0.0 \\
\hline Selected public sector entities balance $3 /$ & 0.3 & 0.2 & 0.0 & 0.0 & 0.0 & 0.0 & 0.0 & 0.0 & 0.0 & 0.0 & 0.0 \\
\hline Primary fiscal balance & -3.3 & -4.2 & -3.7 & -4.7 & -4.1 & -3.7 & -3.4 & -3.0 & -3.0 & -3.0 & -2.9 \\
\hline \multicolumn{12}{|l|}{ Overall fiscal balance } \\
\hline Payment order basis, excluding grants & -6.2 & -7.0 & -7.2 & -8.1 & -7.3 & -6.9 & -6.5 & -6.2 & -6.2 & -6.2 & -6.2 \\
\hline Payment order basis, including grants & -3.7 & -4.6 & -4.5 & -5.1 & -4.9 & -4.5 & -4.3 & -4.0 & -4.0 & -4.0 & -4.0 \\
\hline Basic fiscal balance 4/ & -1.0 & -0.8 & -2.0 & -2.8 & -1.9 & -1.5 & -1.1 & -0.8 & -0.8 & -0.7 & -0.7 \\
\hline Financing & 3.7 & 4.6 & 4.5 & 5.1 & 4.9 & 4.5 & 4.3 & 4.0 & 4.0 & 4.0 & 4.0 \\
\hline External financing & 2.4 & 3.8 & 4.9 & 4.1 & 3.0 & 3.0 & 3.0 & 2.9 & 2.9 & 2.5 & 3.2 \\
\hline Domestic financing & 1.1 & 2.1 & 0.3 & 2.5 & 2.9 & 1.6 & 1.4 & 1.1 & 1.0 & 1.5 & 0.3 \\
\hline Settlement of payment delays $5 /$ & 0.0 & -1.4 & -1.1 & -1.6 & -1.3 & -0.5 & -0.2 & 0.0 & 0.0 & 0.0 & 0.0 \\
\hline Errors and omissions & 0.2 & 0.2 & 0.0 & 0.1 & 0.0 & 0.0 & 0.0 & 0.0 & 0.0 & 0.0 & 0.0 \\
\hline Financing gap 6/ & 0.0 & 0.0 & 0.4 & 0.0 & 0.4 & 0.4 & 0.0 & 0.0 & 0.0 & 0.0 & 0.0 \\
\hline Memorandum items: & \multicolumn{11}{|c|}{ (Percent of GDP, unless otherwise indicated) } \\
\hline Priority expenditure $7 /$ & 8.9 & 8.9 & 9.1 & 9.1 & 9.3 & 9.3 & $\ldots$ & & & & \\
\hline Wages and salaries (percent of revenue) & 28.7 & 30.2 & 31.9 & 32.5 & 32.0 & 32.3 & 31.1 & 30.2 & 29.8 & 29.5 & 29.2 \\
\hline
\end{tabular}

Sources: Senegalese authorities; and IMF staff estimates and projections.

1/ From 2006 on, reflects post-MDRI debt service schedule.

2/ Includes SENELEC recapitalization. The government provided CFAF 65 billion in 2007 under domestically-financed capital expenditure, while earmarked budget support by the World Bank and France in 2008-10 is being provided under 3/ Local governments, autonomous public sector entities (e.g. hospitals, universities), and the civil servants pension fund (FNR).

4/ Defined as total revenue minus total expenditure and net lending, excluding externally financed capital expenditure, on-lending,

HIPC/MDRI expenditure, and 2010 clearing of extrabudgetary spending and agency debt.

5/ Within the expenditure chain in 2008-09 and extrabudgetary spending and agency debt in 2009-11. 6/ Financing gap in 2010 to be filled with ESF drawing.

7/ Defined as expenditures on health, education, environment, the judiciary, social development, sewage, and rural irrigation. 
Table 5. Senegal: Monetary Survey, 2005-10

\begin{tabular}{|c|c|c|c|c|c|c|}
\hline & 2005 & 2006 & 2007 & 2008 & 2009 & 2010 \\
\hline & & & & & Est. & Proj. \\
\hline & \multicolumn{6}{|c|}{ (CFAF billions) } \\
\hline Net foreign assets & 660 & 780 & 851 & 762 & 859 & 868 \\
\hline Central Bank of West African States (BCEAO) 1/ & 487 & 569 & 644 & 653 & 725 & 728 \\
\hline Commercial banks & 173 & 210 & 207 & 109 & 133 & 140 \\
\hline Net domestic assets & 894 & 972 & 1,122 & 1,245 & 1,367 & 1,592 \\
\hline Net domestic credit & 1,032 & 1,122 & 1,324 & 1,467 & 1,604 & 1,825 \\
\hline Net credit to the government & -35 & 11 & 96 & 28 & 112 & 217 \\
\hline Central bank & 84 & 45 & 55 & -14 & 119 & 169 \\
\hline Commercial banks & -123 & -46 & 21 & 33 & -9 & 45 \\
\hline Other institutions & 4 & 12 & 20 & 9 & 2 & 2 \\
\hline Credit to the economy & 1,067 & 1,111 & 1,228 & 1,440 & 1,492 & 1,608 \\
\hline Other items (net) & -138 & -151 & -202 & -223 & -236 & -233 \\
\hline Broad money (M2) & 1,553 & 1,751 & 1,973 & 2,007 & 2,219 & 2,459 \\
\hline Currency outside banks & 378 & 453 & 485 & 474 & 495 & 518 \\
\hline Total deposits & 1,176 & 1,298 & 1,488 & 1,532 & 1,724 & 1,941 \\
\hline Demand deposits & 593 & 652 & 784 & 779 & 857 & 1,022 \\
\hline \multirow[t]{2}{*}{ Time deposits } & 582 & 646 & 705 & 754 & 867 & 919 \\
\hline & \multicolumn{6}{|c|}{ (Change in percentage of beginning-of-period broad money stock) } \\
\hline Net foreign assets & -1.2 & 7.7 & 4.1 & -4.5 & 4.8 & 0.4 \\
\hline BCEAO & 0.7 & 5.3 & 4.3 & 0.4 & 3.6 & 0.1 \\
\hline Commercial banks & -1.8 & 2.4 & -0.2 & -5.0 & 1.2 & 0.3 \\
\hline Net domestic assets & 8.6 & 5.0 & 8.6 & 6.2 & 6.1 & 10.1 \\
\hline Net credit to the government & -4.1 & 3.0 & 4.9 & -3.5 & 4.2 & 4.7 \\
\hline Credit to the economy & 14.5 & 2.9 & 6.7 & 10.7 & 2.6 & 5.2 \\
\hline Other items (net) & -1.8 & -0.8 & -2.9 & -1.0 & -0.7 & 0.1 \\
\hline Broad money (M2) & 7.4 & 12.7 & 12.7 & 1.7 & 10.6 & 10.8 \\
\hline Memorandum items: & \multicolumn{6}{|c|}{ (Units indicated) } \\
\hline Velocity (GDP/M2; end of period) & 3.0 & 2.8 & 2.7 & 3.0 & 2.7 & 2.6 \\
\hline Nominal GDP growth (percentage growth) & 8.3 & 6.5 & 10.5 & 9.8 & 1.1 & 5.8 \\
\hline Credit to the economy (percentage growth) & 24.5 & 4.2 & 10.5 & 17.2 & 3.6 & 7.8 \\
\hline Credit to the economy/GDP (percent) & 23.2 & 22.7 & 22.7 & 24.3 & 24.9 & 25.3 \\
\hline Variation of net credit to the government (from & & & & & & \\
\hline previous year; CFAF billions) & -59.2 & 46.3 & 85.1 & -68.3 & 83.7 & 104.9 \\
\hline Central bank refinance rate (eop/latest; percent) & 4.00 & 4.25 & 4.25 & 4.75 & 4.25 & 4.25 \\
\hline
\end{tabular}

Sources: Senegalese authorities; and IMF staff estimates and projections.

1/ Difference in 2009 between changes in NFA and NIR due to SDR allocation. 
Table 6. Financial Soundness Indicators for the Banking Sector, 2002-09 (Percent, unless otherwise indicated)

\begin{tabular}{|c|c|c|c|c|c|c|c|c|}
\hline & $\begin{array}{l}2002 \\
\text { Dec. }\end{array}$ & $\begin{array}{l}2003 \\
\text { Dec. }\end{array}$ & $\begin{array}{l}2004 \\
\text { Dec. }\end{array}$ & $\begin{array}{l}2005 \\
\text { Dec. }\end{array}$ & $\begin{array}{l}2006 \\
\text { Dec. }\end{array}$ & $\begin{array}{l}2007 \\
\text { Dec. }\end{array}$ & $\begin{array}{l}2008 \\
\text { Dec. }\end{array}$ & $\begin{array}{l}2009 \\
\text { Dec. }\end{array}$ \\
\hline \multicolumn{9}{|l|}{ Capital adequacy } \\
\hline Capital to risk-weighted assets & 16.0 & 11.7 & 11.5 & 10.8 & 12.9 & 13.5 & 13.8 & 16.3 \\
\hline Regulatory capital to risk-weighted assets & 15.5 & 12.1 & 11.9 & 11.1 & 13.1 & 13.6 & 13.9 & 16.5 \\
\hline Capital to total assets & 10.3 & 7.8 & 7.7 & 7.6 & 8.3 & 8.3 & 9.1 & 9.3 \\
\hline \multicolumn{9}{|l|}{ Asset composition and quality } \\
\hline Total loans to total assets & 58.3 & 59.6 & 57.1 & 64.0 & 63.8 & 58.8 & 62.8 & 59.5 \\
\hline $\begin{array}{l}\text { Concentration: loans to } 5 \text { largest borrowers to capital } \\
\text { Sectoral distribution of loans }\end{array}$ & 104.9 & 141.0 & 131.4 & 179.9 & 103.7 & 88.5 & 86.9 & 88.1 \\
\hline Industrial & 36.4 & 41.1 & 33.6 & 35.5 & 28.9 & 25.1 & 19.5 & 27.5 \\
\hline Retail and wholesale trade & 22.2 & 19.9 & 19.3 & 17.0 & 18.9 & 14.4 & 18.5 & 24.5 \\
\hline Services, transportation and communication & 17.5 & 17.2 & 27.4 & 28.0 & 30.0 & 29.6 & 31.1 & 41.0 \\
\hline Gross NPLs to total loans 1/ & 18.5 & 13.3 & 12.6 & 11.9 & 16.8 & 18.6 & 19.1 & 18.7 \\
\hline Of which: without ICS & & & & $\ldots$ & $\ldots$ & 12.7 & 14.2 & 15.8 \\
\hline Provisions to NPLs 1/ & 70.5 & 75.3 & 75.7 & 75.4 & 52.0 & 53.8 & 51.5 & 53.1 \\
\hline Of which: without ICS & & $\ldots$ & $\ldots$ & $\ldots$ & $\ldots$ & 74.6 & 62.9 & 64.7 \\
\hline NPLs net of provisions to total loans $1 /$ & 5.5 & 3.3 & 3.4 & 3.2 & 8.8 & 8.6 & 9.3 & 9.7 \\
\hline Of which: without ICS & & & & & & 3.6 & 5.4 & 6.2 \\
\hline NPLs net of provisions to capital $1 /$ & 30.7 & 27.8 & 25.1 & 27.2 & 67.9 & 60.7 & 63.9 & 62.3 \\
\hline Of which: without ICS & & $\cdots$ & $\cdots$ & $\ldots$ & $\ldots$ & 23.8 & 35.3 & 38.4 \\
\hline \multicolumn{9}{|l|}{ Earnings and profitability } \\
\hline Average cost of borrowed funds & 2.2 & 1.8 & 2.0 & 2.0 & 2.2 & 2.3 & 2.8 & $\ldots$ \\
\hline Average interest rate on loans & 9.7 & 8.7 & 11.7 & 11.8 & 11.3 & 11.6 & 13.9 & $\ldots$ \\
\hline Average interest margin $2 /$ & 7.6 & 6.7 & 9.7 & 9.8 & 9.2 & 9.4 & 11.1 & $\ldots$ \\
\hline After-tax return on average assets & 1.8 & 1.8 & 1.8 & 1.6 & 1.6 & 1.6 & 1.4 & $\ldots$ \\
\hline After-tax return on average equity & 21.1 & 22.1 & 17.6 & 15.8 & 14.6 & 15.3 & 13.0 & $\ldots$ \\
\hline Noninterest expenses/net banking income & 45.5 & 48.9 & 48.7 & 47.9 & 49.4 & 50.7 & 51.3 & $\cdots$ \\
\hline Salaries and wages/net banking income & 20.6 & 21.8 & 21.5 & 21.2 & 21.7 & 22.2 & 21.1 & $\ldots$ \\
\hline \multicolumn{9}{|l|}{ Liquidity } \\
\hline Total deposits to total liabilities & 78.5 & 82.0 & 79.6 & 78.3 & 75.8 & 73.6 & 70.3 & 74.9 \\
\hline
\end{tabular}

Source: BCEAO.

1/ NPL changes in 2006 due to ICS. In 2008, ICS was recapitalized and the government guarantee for its bank loans was lifted. However, the loans in question remain classified as non-performing for the time being, although without the need to provision.

2/ Excluding the tax on banking operations. 
Table 7. Quantitative Assessment Criteria (Performance Criteria) and Indicative Targets for 2009-10 1/ (CFAF billions, unless otherwise specified)

\begin{tabular}{|c|c|c|c|c|c|c|}
\hline & \multicolumn{3}{|c|}{ December 31, 2009} & \multicolumn{3}{|c|}{ March 31, 2010} \\
\hline & $\begin{array}{c}\text { Assessment } \\
\text { criterion }\end{array}$ & Actual & Status & $\begin{array}{c}\text { Indicative } \\
\text { target }\end{array}$ & Actual & Status \\
\hline \multicolumn{7}{|l|}{ Assessment (performance) criteria } \\
\hline Floor on the basic fiscal balance $2 /$ & -119 & -168 & Not met & -30 & 37 & Met \\
\hline $\begin{array}{l}\text { Ceiling on the contracting or guaranteeing of new } \\
\text { nonconcessional external debt by the government } 3 / 4 / \\
\text { Ceiling on spending undertaken outside normal and simplified }\end{array}$ & $905 /$ & $875 /$ & Met & $06 /$ & 0 & Met \\
\hline procedures $4 /$ & 0 & 0 & Met & 0 & 0 & Met \\
\hline $\begin{array}{l}\text { Ceiling on government external payment arrears (stock) 4/ } \\
\text { Ceiling on the amount of the float (depenses liquidées }\end{array}$ & 0 & 0 & Met & 0 & 0 & Met \\
\hline non payées par le Tresor) $7 /$ & 45 & 37 & Met & 45 & 40 & Met \\
\hline \multicolumn{7}{|l|}{ Indicative target } \\
\hline $\begin{array}{l}\text { Quarterly ceiling on the share of the value of public sector } \\
\text { contracts signed by single tender (percent) }\end{array}$ & 20 & 6 & Met & 20 & 15 & Met \\
\hline
\end{tabular}

1/ Indicative targets for March 2010, except for the assessment (performance) criteria monitored on a continuous basis. 2/ Defined as total revenue minus total expenditure and net lending, excluding externally financed capital expenditure, on-lending, and HIPC and MDRI spending. Cumulative since the beginning of the year. Total revenue excludes privatization receipts and sales of mobile telephone licenses.

3/ Excluding government or government-guaranteed CFAF borrowing from financial institutions within the WAEMU and external loans contracted by the airport project company (AIDB) to finance the construction of the new Dakar Airport. 4/ Monitored on a continuous basis.

5/ Cumulative since approval of second PSI review. The amount raised from CFAF 80 billion to CFAF 90 billion at the 4 th review is to finance exclusively the Dakar-Diamniadio toll highway project.

6/ Cumulative since approval of fourth PSI review.

7/ Defined as all expenditure for which a bill has been received and recognized (dépense liquidée ) but not yet paid by the Treasury. 
Table 8. Senegal: Indicators of Capacity to Repay the Fund, 2008-20

\begin{tabular}{|c|c|c|c|c|c|c|c|c|c|c|c|c|}
\hline & 2009 & 2010 & 2011 & 2012 & 2013 & 2014 & 2015 & 2016 & 2017 & 2018 & 2019 & 2020 \\
\hline & & & & & Projec & & & & & & & \\
\hline \multicolumn{13}{|l|}{ Obligations to the Fund from existing drawings } \\
\hline Principal (SDR millions) & 0.0 & 0.4 & 2.1 & 3.5 & 3.5 & 11.6 & 20.9 & 19.2 & 17.8 & 17.8 & 9.7 & 0.0 \\
\hline Charges/Interest (SDR millions) 1/ & 0.2 & 0.1 & 0.1 & 0.3 & 0.3 & 0.3 & 0.2 & 0.2 & 0.1 & 0.1 & 0.0 & 0.0 \\
\hline \multicolumn{13}{|l|}{ Obligations to the Fund from prospective drawings $2 /$} \\
\hline Principal (SDR millions) & 0.0 & 0.0 & 0.0 & 0.0 & 0.0 & 0.0 & 3.2 & 6.5 & 6.5 & 6.5 & 6.5 & 3.2 \\
\hline Charges/Interest (SDR millions) 1/ & 0.0 & 0.0 & 0.0 & 0.1 & 0.1 & 0.1 & 0.1 & 0.1 & 0.1 & 0.0 & 0.0 & 0.0 \\
\hline \multicolumn{13}{|l|}{ Total existing and prospective obligations to the Fund $2 /$} \\
\hline In millions of SDRs & 0.2 & 0.4 & 2.2 & 3.8 & 3.8 & 11.9 & 24.5 & 25.9 & 24.4 & 24.4 & 16.2 & 3.3 \\
\hline In millions of U.S. dollars & 0.3 & 0.6 & 3.3 & 5.9 & 5.9 & 18.3 & 37.5 & 39.7 & 37.5 & 37.4 & 24.9 & 5.0 \\
\hline In percent of exports of goods and services & 0.0 & 0.0 & 0.1 & 0.2 & 0.1 & 0.4 & 0.8 & 0.8 & 1.1 & 1.0 & 0.6 & 0.1 \\
\hline In percent of debt service & 0.2 & 0.4 & 1.4 & 2.4 & 2.4 & 4.7 & 15.6 & 15.6 & 14.1 & 13.4 & 9.2 & 1.7 \\
\hline In percent of quota & 0.1 & 0.3 & 1.3 & 2.4 & 2.4 & 7.4 & 15.1 & 16.0 & 15.1 & 15.1 & 10.0 & 2.0 \\
\hline In percent of gross official reserves & 0.1 & 0.0 & 0.2 & 0.3 & 0.3 & 0.8 & 1.4 & 1.4 & 1.3 & 1.2 & 0.7 & 0.1 \\
\hline \multicolumn{13}{|l|}{ Total Fund credit outstanding $2 /$} \\
\hline In millions of SDRs & 106.3 & 138.3 & 136.3 & 132.8 & 129.3 & 117.8 & 93.6 & 68.0 & 43.7 & 19.4 & 3.2 & 0.0 \\
\hline In millions of U.S. dollars & 168.2 & 211.6 & 208.4 & 203.4 & 198.2 & 180.7 & 143.6 & 104.3 & 67.0 & 29.8 & 5.0 & 0.0 \\
\hline In percent of exports of goods and services & 5.7 & 6.5 & 6.0 & 5.5 & 5.0 & 4.3 & 3.2 & 2.1 & 1.9 & 0.8 & 0.1 & 0.0 \\
\hline In percent of debt service & 104.2 & 122.7 & 89.1 & 84.7 & 82.6 & 46.1 & 59.5 & 40.9 & 25.2 & 10.7 & 1.8 & 0.0 \\
\hline In percent of quota & 65.7 & 85.5 & 84.2 & 82.1 & 79.9 & 72.8 & 57.9 & 42.0 & 27.0 & 12.0 & 2.0 & 0.0 \\
\hline In percent of gross official reserves & 7.9 & 10.5 & 10.1 & 9.5 & 8.7 & 7.4 & 5.5 & 3.8 & 2.3 & 1.0 & 0.1 & 0.0 \\
\hline \multicolumn{13}{|l|}{ Memorandum items: } \\
\hline Exports of goods and services (millions of US\$) & 2,962 & 3,271 & 3,467 & 3,683 & 3,957 & 4,229 & 4,522 & 4,881 & 3,455 & 3,655 & 3,868 & 4,093 \\
\hline Debt service (millions of US\$) & 161 & 172 & 234 & 240 & 240 & 392 & 241 & 255 & 266 & 279 & 272 & 293 \\
\hline Quota (millions of SDRs) & 162 & 162 & 162 & 162 & 162 & 162 & 162 & 162 & 162 & 162 & 162 & 162 \\
\hline Gross official reserves (billions of US\$) & 2.1 & 2.0 & 2.1 & 2.1 & 2.3 & 2.4 & 2.6 & 2.8 & 2.9 & 3.1 & 3.4 & 3.6 \\
\hline GDP (millions of US\$) & 12,738 & 13,176 & 13,872 & 14,730 & 15,662 & 16,682 & 17,797 & 19,103 & 20,514 & 22,031 & 23,670 & 25,438 \\
\hline
\end{tabular}

Sources: BCEAO; and Fund staff estimates and projections.

1/ Reflects temporary waiver of interest payments on concessional lending in 2010-11. No changes have been made in charges related to the SDR department. 2/ Based on an ESF disbursement of SDR 32.36 million (20 percent of quota) upon completion of the third ESF review, May 2010. 
Table 9: Millennium Development Goals 1/

\begin{tabular}{|c|c|c|c|c|c|}
\hline & 1990 & 1995 & 2000 & 2005 & 2008 \\
\hline \multicolumn{6}{|c|}{2015 target: Halve 1990 US $\$ 1$ /day poverty and malnutrition rate } \\
\hline \multicolumn{6}{|l|}{ Goal 1: Eradicate extreme poverty and hunger } \\
\hline Employment to population ratio, $15+$, total $(\%)$ & 67 & 67 & 66 & 65 & 66 \\
\hline Employment to population ratio, ages $15-24$, total (\%) & 59 & 59 & 57 & 55 & 54 \\
\hline GDP per person employed (annual \% growth) & -2 & 3 & 0 & 3 & 1 \\
\hline Income share held by lowest $20 \%$ & 3.5 & 6.5 & 6.6 & 6.2 & .. \\
\hline Malnutrition prevalence, weight for age ( $\%$ of children under 5 ) & .. & 21.9 & 20.3 & 14.5 & .. \\
\hline Poverty gap at $\$ 1.25$ a day (PPP) (\%) & 34 & 19 & 14 & 11 & .. \\
\hline Poverty headcount ratio at $\$ 1.25$ a day (PPP) (\% of population) & 66 & 54 & 44 & 34 & .. \\
\hline Prevalence of undernourishment (\% of population) & 28 & 32 & .. & 26 & .. \\
\hline Vulnerable employment, total (\% of total employment) & 83 & .. & .. & .. & .. \\
\hline \multicolumn{6}{|l|}{2015 target: Net enrollment to 100} \\
\hline \multicolumn{6}{|l|}{ Goal 2: Achieve universal primary education } \\
\hline Literacy rate, youth female (\% of females ages $15-24)$ & 28 &.. & 41 & 45 & 45 \\
\hline Literacy rate, youth male (\% of males ages $15-24)$ & 49 & .. & 58 & 58 & 58 \\
\hline Persistence to last grade of primary, total (\% of cohort) & .. &.. & 63 & 53 & .. \\
\hline Primary completion rate, total (\% of relevant age group) & 43 & 38 & 38 & 51 & 50 \\
\hline Total enrollment, primary (\% net) & .. & .. & 57 & 73 & 73 \\
\hline \multicolumn{6}{|l|}{2015 target: Education ratio to 100} \\
\hline \multicolumn{6}{|l|}{ Goal 3: Promote gender equality and empower women } \\
\hline Proportion of seats held by women in national parliaments (\%) & 13 & 12 & 12 & 19 & 22 \\
\hline Ratio of female to male enrollments in tertiary education &.. &.. & .. & 46 & 55 \\
\hline Ratio of female to male primary enrollment & 73 & 76 & 86 & 96 & 100 \\
\hline Ratio of female to male secondary enrollment & 53 & .. & 65 & 75 & 76 \\
\hline $\begin{array}{r}\text { Share of women employed in the nonagricultural sector (\% of total nonagricultural employment) } \\
2015 \text { target: Reduce } 1990 \text { mortality by two-thir }\end{array}$ & .. &.. & 10.6 & .. & .. \\
\hline \multicolumn{6}{|l|}{ Goal 4: Reduce child mortality } \\
\hline Immunization, measles (\% of children ages $12-23$ months) & 51 & 80 & 48 & 74 & 84 \\
\hline Mortality rate, infant (per 1,000 live births) & 72 & 72 & 66 & 61 & 59 \\
\hline Mortality rate, under-5 (per 1,000$)$ & 149 & 148 & 133 & 119 & 114 \\
\hline \multicolumn{6}{|c|}{2015 target: Reduce 1990 maternal mortality by three-fourths } \\
\hline \multicolumn{6}{|l|}{ Goal 5: Improve maternal health } \\
\hline Adolescent fertility rate (births per 1,000 women ages $15-19$ ) &.. & 114 & 109 & 105 & 104 \\
\hline Births attended by skilled health staff (\% of total) &.. & 47 & 60 & 52 & .. \\
\hline Contraceptive prevalence (\% of women ages $15-49$ ) & .. & 13 & 11 & 12 & .. \\
\hline Maternal mortality ratio (modeled estimate, per 100,000 live births) & .. &.. & .. & 980 & .. \\
\hline Pregnant women receiving prenatal care $(\%)$ & .. & 82 & 79 & 87 & .. \\
\hline Unmet need for contraception (\% of married women ages $15-49)$ & .. & 35 & .. & 32 & .. \\
\hline \multicolumn{6}{|c|}{2015 target: Halt and begin to reverse, AIDS and other major diseases } \\
\hline \multicolumn{6}{|c|}{ Goal 6: Combat HIVIAIDS, malaria, and other diseases } \\
\hline Children with fever receiving antimalarial drugs ( $\%$ of children under age 5 with fever) & .. &.. & 36 & 27 & 22 \\
\hline Condom use, population ages $15-24$, female (\% of females ages $15-24)$ & .. & .. & .. & 5 & .. \\
\hline Condom use, population ages $15-24$, male $(\%$ of males ages $15-24)$ & .. &.. & .. & 48 & .. \\
\hline Incidence of tuberculosis (per 100,000 people) & 195 & 215 & 237 & 261 & 272 \\
\hline Prevalence of HIV, female (\% ages $15-24)$ & .. & .. & .. & 0.8 & 0.8 \\
\hline Prevalence of HIV, male ( $\%$ ages $15-24)$ & .. & .. & .. & 0 & 0 \\
\hline Prevalence of HIV, total (\% of population ages $15-49$ ) & 0.1 & 0.2 & 0.4 & 0.8 & 1.0 \\
\hline Tuberculosis cases detected under DOTS (\%) & .. & 62 & 53 & 50 & 48 \\
\hline
\end{tabular}

2015 target: Various, of which halve the percentage of population with no durable access to drinking water

Goal 7: Ensure environmental sustainability

$\mathrm{CO} 2$ emissions ( $\mathrm{kg}$ per PPP \$ of GDP)

$\mathrm{CO} 2$ emissions (metric tons per capita)

Forest area (\% of land area)

Improved sanitation facilities (\% of population with access)

Improved water source (\% of population with access)

Marine protected areas, ( $\%$ of surface area)

Nationally protected areas (\% of total land area)

Goal 8: Develop a global partnership for development

Aid per capita (current US\$)

Debt service (PPG and IMF only, \% of exports, excluding workers' remittances)

Internet users (per 100 people)

Mobile cellular subscriptions (per 100 people)

Telephone lines (per 100 people)

Other

Fertility rate, total (births per woman)

GNI per capita, Atlas method (current US\$)

GNI, Atlas method (current US\$) (billions)

Gross capital formation (\% of GDP)

Life expectancy at birth, total (years)

Literacy rate, adult total (\% of people ages 15 and above)

Population, total (millions)

Trade $(\%$ of GDP)

$\begin{array}{rrrrr}0.4 & 0.4 & 0.3 & 0.3 & . . \\ 0.4 & 0.4 & 0.4 & 0.4 & . . \\ 49 & 47 & 46 & 45 & . . \\ 26 & 27 & 27 & 28 & 28 \\ 67 & 69 & 72 & 77 & 77 \\ . . & . . & . . & 0 & . . \\ . . & . . & . . & 11.2 & 11.2 \\ & & & & \\ 108 & 76 & 43 & 61 & 71 \\ 18 & 16 & 13 & 6 & 4 \\ 0.0 & 0.0 & 0.4 & 4.8 & 8.4 \\ 0 & 0 & 3 & 15 & 44 \\ 1 & 1 & 2 & 2 & 2 \\ & & & & \\ 6.7 & 6.1 & 5.6 & 5.2 & 5.0 \\ . . & . . & . . & 800 & 970 \\ . . & . . & . . & 9.3 & 11.8 \\ 9.1 & 13.6 & 20.5 & 29.7 & 30.2 \\ 52 & 53 & 54 & 55 & 56 \\ 27 & . . & 39 & 42 & 42 \\ 7.5 & 8.7 & 9.9 & 11.3 & 12.2 \\ 57.6 & 68.2 & 65.1 & 69.5 & 72.4 \\ & & & & \end{array}$

Source: World Development Indicators database and Senegalese authorities for the 2015 targets ("Rapport de suivi des OMD", April 2010).

$1 /$ Figures in italics refer to periods other than those specified. 


\section{APPENDIX I \\ LETTER OF INTENT}

Dakar, Senegal

May 7, 2010

Mr. Dominique Strauss-Kahn

Managing Director

International Monetary Fund

$70019^{\text {th }}$ Street, N.W.

Washington, DC 20431

Dear Mr. Strauss-Kahn:

1. The attached Memorandum of Economic and Financial Policies (MEFP) reviews recent economic developments in Senegal and implementation of the government of Senegal's macroeconomic program under the three-year Policy Support Instrument (PSI), which was approved by the IMF Executive Board on November 2, 2007, and the Exogenous Shocks Facility (ESF) arrangement, approved on December 19, 2008 and augmented and extended on June 19, 2009. The attached MEFP builds on previous MEFPs and sets out the policies the government intends to implement, with emphasis on measures and objectives for the remainder of 2010. The ESF arrangement will expire on June 18, 2010 and the PSI on November 1, 2010.

2. Most of the quantitative assessment/performance criteria for end-December 2009 and all quantitative indicative targets for end-March 2010 were met. The target for the basic fiscal balance was exceeded by 0.8 percent of GDP, which is explained in part by revenues that were lower than projected at the time of the last review and by increased spending to meet urgent needs. In the future, the government will endeavor to offset higher-than-budgeted spending by restricting nonpriority expenditures. It is requesting a waiver for exceeding the ceiling on the basic fiscal deficit. The government is determined to pursue sound policies and to bring the overall fiscal deficit down to 4 percent of GDP in the medium term.

3. Structural reforms, which are focused on fiscal management, governance, and transparency, and the preparation of an action plan for the financial sector, have generally progressed in line with the program despite some delays. Given the complexity of the task, the development of procedures for the regularization of extrabudgetary commitments has taken more time than expected. On the other hand, in the interim the government has paid some agency debt and some extrabudgetary spending has already been incorporated in the budget. The government will present a supplementary budget to Parliament by May 15, 2010 that will contain specific line items to settle these payments. Debts that do not require a budgetary allocation will be presented in an annex to the law. 
As indicated in the attached MEFP, the authorities are continuing to pursue an ambitious program of budgetary and financial reforms, as well as governance and energy sector reforms.

4. In light of overall program performance and based on the policies set forth in the attached MEFP, the government requests completion of the fifth review under the PSI and the third and final review under the ESF arrangement with a disbursement in the amount of SDR 32.36 million.

5. The government believes that the policies and measures set forth in the attached MEFP are sufficient to achieve the objectives of the PSI-supported program during the remaining months. It will promptly take any additional measures necessary for the achievement of the objectives of the program. The government will consult with the IMF - at its own initiative or whenever the Managing Director of the IMF requests such consultation - before adopting any such measures or changes to the policies described in the attached MEFP. The government will provide the IMF with such information as it may request in connection with the progress made in implementing the economic and financial policies and achieving the objectives of the program. It is expected that the sixth and final review under the PSI will be completed by end-October, 2010, as the instrument will expire on November 1, 2010. During the next review mission, the government intends to examine with the IMF staff the possibility of another program.

6. The government authorizes the IMF to publish this letter, the attached MEFP, and the related Staff Report, which also includes the Article IV consultation report and the Debt Sustainability Analysis.

\author{
Sincerely yours, \\ /s/ \\ Abdoulaye Diop \\ Minister of State \\ Minister of Economy and Finance
}

Attachments: - Memorandum of Economic and Financial Policies (MEFP)

-Technical Memorandum of Understanding (TMU) 


\title{
ATTACHMENT I
}

\section{Memorandum of Economic and Financial Policies}

\author{
Dakar, May 7, 2010
}

\section{INTRODUCTION}

1. This memorandum updates Senegal's economic program under the threeyear Policy Support Instrument (PSI) for the period 2007-2010 and the Exogenous Shocks Facility (ESF) arrangement approved in December 2008. ${ }^{1}$ The program supported under the PSI focuses on: (i) following a prudent fiscal policy and avoiding a recurrence of payment delays; (ii) strengthening governance and transparency; (iii) promoting private sector activity; and (iv) enhancing the contribution of the financial sector to the economy. This Memorandum describes recent economic developments and program implementation, and discusses the economic outlook for the rest of 2010 and the government's commitments regarding ongoing macroeconomic and structural reforms.

2. The government remains committed to meeting the objectives of the program supported by the PSI. It aims to preserve macroeconomic stability, foster good fiscal management and governance, strengthen growth driven by the private sector, and make rapid progress toward achieving the Millennium Development Goals (MDGs). In collaboration with the IMF, the government has demonstrated that it is determined to overcome the budget execution problems encountered in 2008. Decisive improvements were made in correcting payment delays to the private sector and implementing ambitious reforms to improve the efficiency and transparency of fiscal management. The settlement of extrabudgetary expenditures and public institution and agency debt still outstanding in 2010 and 2011 should make it possible to fully normalize relations with the private sector. This will also make it possible to broaden the focus of reform in Senegal.

\section{RECENT ECONOMIC DEVELOPMENTS, SHORT-TERM OUTLOOK, AND Program Performance}

3. Macroeconomic performance remains below trend. The global financial crisis had a marked impact on Senegal's economy but domestic factors also played a role.

- $\quad$ Real GDP growth was modest. In 2009, business activity remained weak. The general activity index (excluding agriculture and government) was flat for most of 2009 (year-on-year), but there was a modest upturn in activity in the last quarter. Estimated growth was around $1 \frac{1}{2}$ percent in 2009 . The relatively robust performance of the primary sector is attributable primarily to favorable climatic conditions and a more dynamic agricultural policy. In the secondary sector, Industries Chimiques du Sénégal (ICS) continues to recover but was faced with

\footnotetext{
${ }^{1}$ Details of this program were set out in the initial MEFP of October 3, 2007 and in the MEFPs of May 30, 2008, December 5, 2008, June 5, 2009, and December 4, 2009.
} 
significantly lower export prices. Results in the tertiary sector, a traditional engine of growth in the Senegalese economy, were poor.

- The financial and operational difficulties of SENELEC (the public electricity company) led to repeated power cuts that were detrimental to production and investment by private sector enterprises.

- Inflation measured by the consumer price index (year-on-year) has been negative since May 2009, largely because of a decline in food and energy prices.

- $\quad$ The external current account deficit is estimated to have improved compared to 2008 , primarily as a result of the decline in oil and food prices for the year as a whole.

\section{Most quantitative program criteria for end-December 2009 were met.}

(a) The ceiling on the basic fiscal deficit was exceeded by 0.8 percent of GDP in 2009. The basic fiscal deficit at end-December 2009 was CFAF 168 billion, while the ceiling set in the program was CFAF 119 billion. Tax revenues from public entities were lower than anticipated as they accumulated tax arrears. At the same time, current spending was higher than expected in response to urgent needs. These expenditures were based on budget appropriations authorized through supplemental appropriation decrees. Current spending without firm spending limits in the budget, such as telephone, electricity, and water charges, was also higher than expected. However, the government continued to develop its investment program and capital spending reached $10^{1 / 2}$ percent of GDP. The government will endeavor to limit recourse to supplemental appropriation decrees and to better estimate spending that does not have firm spending limits in the budget, and it notes that it has restricted this type of spending starting with the 2010 budget. In the future, the government will endeavor to offset budgetary expenditure overruns by reducing nonpriority spending and will transmit all supplemental appropriation decrees to Parliament in a timely manner. In accordance with applicable law, the government will ratify supplemental appropriation decrees in the following initial or supplementary budget. Given the need to support demand in a very difficult external context and the measures that the government has taken, the government will request a waiver for exceeding the ceiling on the fiscal deficit. According to preliminary data, the (indicative) target for the basic fiscal balance at end- March 2010 was met.

(b) At end-December 2009, the budgetary float had declined to a normal level of CFAF 37 billion ( $2 / 3$ percent of GDP) as compared to CFAF 175 billion ( 3 percent of GDP) in October 2008. At end-March 2010, the budgetary float amounted to CFAF 40 billion, below the (indicative) ceiling of CFAF 45 billion.

(c) The government did not accumulate any external payment arrears. 
(d) In December 2009, the government successfully launched an international bond issue in the amount of US\$200 million (CFAF 87 billion) to finance the DakarDiamniadio highway, authorized under the program for up to CFAF 90 billion. The issue was oversubscribed. The five-year bond bears a nominal interest rate of 8.75 percent and an effective interest rate of 9.25 percent.

(e) The government has not approved any Treasury cash advances since end-2008 and has executed budget expenditure according to the normal and simplified procedures. As indicated above, in 2009 the government used supplemental appropriation decrees, in accordance with the organic law of 2001 and signed by the President of the Republic and the Prime Minister, to establish new budget appropriations for expenditures totaling CFAF 30 billion, approximately half of which were used to finance the wages of contractual workers in the education sector in order to avoid widespread strikes.

(f) The government limited single-tender contracts to 6 percent of all government procurement in the fourth quarter of 2009 and 15 percent in the first quarter of 2010 (compared to a program ceiling of 20 percent).

\section{On the whole, structural reforms continued in line with program commitments. Most of the structural benchmarks were met:}

(g) As last year, the government followed applicable budget execution and accounting closure rules. The cutoff date for budget commitments was November 30 and only a few minor commitments were contracted during the early part of December. The complementary period provided in the law, which ends the administrative phase of budget execution (payment orders) at December 31 (structural benchmark), was essentially respected. To the extent allowed by the available information, Treasury suspense accounts were closed at the end of February to reduce the volume of pending operations to the strict minimum at that time. After February 28, any operations from the previous fiscal year affecting the government's cash flow were charged to the current year. The data for fiscal year 2009 in the expenditure tracking system (SIGFIP) were finalized as of April 30, 2010. To achieve greater transparency, the provisional end-ofmonth data from SIGFIP and the government financial operations table (TOFE) were published on the website of the Ministry of Economy and Finance 10 and 45 days, respectively, after the end of the month, beginning with the November 2009 TOFE and the end-December 2009 SIGFIP.

(h) The government has issued a press release summarizing the results of the audit of extrabudgetary expenditure and public institution and agency debt, setting forth its general strategy for settling payments in the first supplementary budget for 2010 (structural benchmark, January 31, 2010). Following the audit by the Office of the Inspector-General of Finance (IGF), an independent external audit of extrabudgetary expenditure completed in June 2009 identified extrabudgetary spending in the amount of 
CFAF 37 billion and public agency and institution debt of CFAF 67 billion as of December 31, 2008. Given the complexity of the task, more time was needed to determine the amount to be settled and to establish the detailed terms and conditions.

(i) The government has taken action following its study of tax expenditures, which revealed that tax expenditures and other types of tax exemption could exceed 3 percent of GDP. Bearing in mind the costs and benefits of specific tax expenditures, the government has established a plan of action identifying the key measures that could be eliminated and examining the legislative and regulatory amendments needed to eliminate them (structural benchmark, March 2010).

(j) The government has reviewed progress with the reform of the financial sector since the 2004 update of the 2001 national Financial Sector Assessment Program (FSAP) and the 2007 regional FSAP. The action plan for Senegal builds on existing plans and measures, identifies the reform manager, distributes the main tasks, and provides an indicative calendar for the reforms (structural benchmark, end-March 2010). The action plan is wide-ranging and includes measures in the following areas: banking and insurance sectors, microfinance institutions and other financial intermediaries, cash flow management, legal framework, and access to financial services.

(k) A supplementary budget will be submitted to Parliament no later than May 15 (structural benchmark). It will include the specific line items authorizing the Ministry of Finance to settle in 2010 the extrabudgetary arrears of ministries and public institutions and agencies and part of the public institution and agency debt identified in the July 2009 audit and not yet budgeted for. Debts not requiring a budgetary allocation will be presented in an annex to the supplementary budget. A detailed analysis conducted by the government has shown that in the interim some extrabudgetary expenditures have been included in the 2009 and 2010 budgets, all required transfers have been made to the public institutions and agencies, and these institutions now have full access to their Treasury deposits. Nevertheless, despite this progress, public institutions still have debts to the private sector, as well as tax and social security contribution debts, because of their own budgetary imbalances.

\section{Macroeconomic Policies For the Remainder of 2010 AND FOR 2011}

\section{A. Outlook}

\section{Economic activity in Senegal remains weak, but signs of recovery are} appearing and the gradual upturn in the global economy should help to stimulate domestic activity. Growth of about $3 \frac{1}{2} 2$ percent is expected in 2010, and inflation should return to about 2 percent as oil prices recover.

7. Uncertainties remain high. A more subdued international recovery than anticipated could slow remittances, official development assistance, exports, and foreign 
direct investment and have a negative impact on growth. Continued implementation of energy sector reform is essential to minimize the risks that repeated power cuts pose for economic activity.

\section{B. Fiscal and Financial Policy Stance}

8. The 2010 budget aims to reconcile several objectives: provide some support for overall demand, protect priority spending, and ensure debt sustainability. Given the impact on revenue of a growth outlook that is slightly below forecasts at the time the budget was prepared, high current spending, and financing constraints, the budget deficit (including grants) will need to be reduced somewhat by restricting spending for a total amount of CFAF 35 billion. Social spending ministeries (education and health) are not affected by this adjustment. The overall budget deficit (including grants) would be about $4 \frac{1}{2}$ percent of GDP in 2010 , which is slightly below the level foreseen during the last review. The revised ceiling for the basic fiscal deficit in 2010 is CFAF 97 billion (CFAF 49 billion at end-June 2010 - assessment criterion). However, in case privatization receipts occur in 2010, spending will correspondingly be reinstated up to the level in the initial budget for 2010. If privatization receipts occur in 2010, spending will be increased and the ceiling on the basic fiscal deficit correspondingly raised by up to CFAF 35 billion.

9. Given that the supplementary budget based on the macroeconomic framework prepared with IMF staff will be submitted to Parliament no later than May 15 (structural benchmark) along with the press release, the government confirms that it is committed to rapidly and transparently settling all extrabudgetary arrears and public institution and agency debt. According to the precise assessment prepared by the authorities, extrabudgetary expenditure in the amount of CFAF 30 billion and public institution and agency debt to the private sector in the amount of CFAF 16 billion remain to be cleared, of which at least 50 percent will be cleared by end-September 2010, in line with the commitment undertaken earlier to settle at least 50 percent of extrabudgetary arrears and public institution and agency debt identified in the July 2009 audit by end-September 2010 (structural benchmark, endSeptember 2010).

10. The government will ask for another internal audit by the Office of the Inspector-General of Finance to verify that no new extrabudgetary commitments or new public institution and agency debt have been accumulated since end-2008. The audit will assess the level of the debt at end-2009. The government will publish a new press release at end-September 2010 that will report on the results of the audit and summarize progress made in clearing the extrabudgetary commitments and public institution and agency debt, to the extent that all claims have not been regularized, and that will detail the next steps to complete this process (structural benchmark, endSeptember 2010). 
11. As part of the government's objectives, the composition of expenditure will receive greater attention. Social spending will increase from 33 percent of total spending in 2008 to almost 35 percent in 2010. The government intends to increase these expenditures to 40 percent of GDP in the near future (the initial PRSP target for 2010 having proven to be overly ambitious). Spending on poverty reduction will be regularly monitored. To support the ongoing development of Senegal's physical infrastructure, which remains one of the government's key objectives, current expenditure on goods and services and transfers will be reduced as a percentage of GDP in 2010. This will free resources that will be used to finance a higher level of domestically financed capital expenditure.

\section{The government bases its medium-term fiscal policy stance on debt} sustainability considerations and will continue to pursue a prudent fiscal policy. It is committed to limiting the overall fiscal deficit to 4 percent of GDP over the medium term and to approximately 3.5 percent of GDP in the longer term. The government's semiannual public debt sustainability analysis will include a risk assessment for contingent liabilities arising from guarantees issued by the government to Public-Private Partnerships (PPPs) and operations of public enterprises.

\section{To ensure debt sustainability, the government will continue to favor} concessional financing and, in general, will not contract or guarantee any new external borrowing on nonconcessional terms. The government will consult with IMF staff in advance regarding any exceptions. Nonconcessional borrowing should be guided by debt sustainability considerations and should be linked to specific economically profitable projects for which sufficient external concessional financing or domestic and regional financing could not be mobilized.

\section{In the future, the government hopes to continue its policy of developing} infrastructure and to benefit from the flexibility provided by the new rules on external debt. It is working on new, large infrastructure projects, including the extension of the Dakar-Diamniadio highway. The government will work in close collaboration with its development partners to assess the economic profitability of these projects and identify the necessary financing. It is planning to have an assessment ready before the next PSI review so that concessional resources and domestic and regional financing may be supplemented, if necessary, with other nonconcessional resources as a last resort. The government is aware that any nonconcessional financing must be linked to economically profitable projects (evaluated by a reliable entity such as the World Bank) and should not jeopardize debt sustainability. 


\section{Structural Reforms}

\section{Budget reforms}

15. To build on progress already made, the government remains committed to continuing its ambitious budget reforms. These reforms, based on an overall fiscal reform program supported by the development partners (in particular the IMF technical assistance report and the PEFA report), are essential to enhancing budget efficiency and transparency, while also helping to protect macroeconomic stability.

\section{As indicated above, the government will endeavor to limit recourse to} supplemental appropriation decrees. The government will also endeavor to maintain budgeted spending levels by offsetting expenditure overruns through equivalent reductions in other spending and will transmit all supplemental appropriation decrees to Parliament in a timely manner. In accordance with applicable law, the government will ratify supplemental appropriation decrees in the following initial or supplementary budget.

\section{The government has taken important steps to prevent the recurrence of} public institution and agency debt. It has based accountants in the public institutions and agencies to track budget execution and regularly report to the Treasury on the liquidity situation. To bolster agency cash flow, the government will ensure the timely processing (no later than 90 days after the Treasury receives the check) of transfers to the accounts of Treasury correspondents (particularly public institutions and agencies) and/or payments to enterprises for invoices by withdrawals from the correspondents' deposit accounts with the Treasury. To this end, the following measures will be applied: (i) a cash flow plan is established in advance between the Treasury and the correspondents and this plan is updated regularly; (ii) the Treasury is notified of transfer or payment intentions at least thirty days before the withdrawal or payment date.

\section{The government will continue to improve SIGFIP as a key budget} monitoring system and will implement the recommendations of the IMF technical assistance missions in the area of public financial management. Following the audit

of SIGFIP and the two technical assistance missions in late 2009 that focused essentially on the expenditure chain and budget execution, a follow-up technical assistance mission took place in late February 2010. The government has made progress using SIGFIP and ASTER as complementary information systems to improve the preparation, execution, and tracking of fiscal and accounting operations and making the government financial operations table (TOFE) more reliable. In the short term the government intends to improve SIGFIP by incorporating payroll expenditure and implementing the SIGFIPASTER interface (structural benchmark, end-July 2010). Work on implementing the interface in the ASTER-SIGFIP direction continues. The interface will strengthen the linkages between fiscal and accounting operations and facilitate preparation of the 
general account of the financial administration, thereby shortening the time required to prepare the final budget review laws (lois de règlement). Moreover, the auxiliary expenditure accounting module (CAD) will be operational in 2010. To press ahead with these reforms, a high-level information systems steering committee has been established within the Ministry of Economy and Finance.

19. The preparation of draft budget review laws will continue. The draft budget review law for 2008 was forwarded to the Audit Court by end-December 2009 and the one for 2009 will be forwarded to the Audit Court by end-September 2010.

20. To improve its cash management, the government is updating the 2006 census of all government accounts, including public institution and agency accounts, with a view to finalizing the strategy to establish a single Treasury account. All government accounts and public institution and agency accounts, including their bank accounts, will be listed. For each account the list will include the number, name of the holder, and the balances as at December 31, 2008 and 2009 (structural benchmark, July 2010). To ensure that the census is comprehensive, the government will ask ministries and public institutions and agencies for a list of accounts and authorization for their banks to provide the necessary information to the Treasury. The census is a significant step toward establishing a single Treasury account, which is an essential tool for improving the central government's daily liquidity management. The government also intends to strengthen liquidity tracking and forecasting at the Treasury by using all available information and requiring the cooperation of all government entities concerned.

21. The government will also improve its debt management. It recognizes the importance of collecting all the necessary information and involving all units concerned. The government intends to better coordinate the tracking of financing needs and management of the government's external and domestic debt, in line with the guidelines given by the National Public Debt Committee. All data on the public debt will be centralized whatever the origin of the loans contracted. In this way, the government envisages establishing a global debt management strategy. These measures could also help to improve indicators of public finance management capacity, such as the PEFA (Public Expenditure and Fiscal Accountability) assessments and CPIA (Country Policy and Institutional Assessments). To improve debt management, the government is also regularly updating the rolling two-year calendar for the issuance of public securities, either quarterly or after each issuance, in cooperation with the Central Bank of West African States (BCEAO).

\section{The process of evaluating and selecting public investment projects will be}

improved. It will be based on the Prime Minister's circular of November 2008 and the commitments undertaken in the initial MEFP (para. 25) and the MEFP for the first review. New projects involving CFAF 500 million or more will be subject to a formal economic cost-benefit/profitability analysis on which the selection process will be based. 
The government also plans to improve the method of determining the order of priority of projects. The government notes that the new airline that has just been created is a majority privately owned company that will not benefit from additional funds or guarantees from the government. Moreover, Air Sénégal International's cessation of operations has thus far required no financing from the government and no supplementary financing is expected.

\section{Tax administration}

23. Following the analysis of tax expenditures, the government prepared an action plan designed to eliminate distortionary tax expenditures with significant budgetary cost. For simplicity and effectiveness, the government will consolidate all legislation relating to domestic taxation in a single document by October 15, 2010 (structural benchmark, October 15, 2010). The government has also committed to conduct an ex ante impact evaluation prior to any adoption of new tax expenditures. This evaluation will be designed to rigorously analyze a measure's likely economic consistency, efficiency, and effectiveness and to reject it if these criteria are not met.

\section{Energy sector}

24. Progress has been made since the last review with the ten measures set out by the government, in particular the implementation of the reform of SENELEC which is progressing satisfactorily, and also the following measures that are in the process of being carried out: strengthening the commercial performance of SENELEC and the process to penalize fraudulent activities, implementing the recommendations of the audit on accounting procedures, and implementing debt restructuring, cancellation, and rescheduling.

The preliminary financial results of SENELEC for 2009 are below the forecasts made in November 2009. The government intends to take the following measures in the shortterm:

- $\quad$ Adjust tariffs, if necessary, effective April 1, complementing the measures indicated above, to reduce the difference between tariffs and variations in production costs or the cost of energy purchases;

- Given SENELEC's tax arrears in 2009, ensure that tax payments by SENELEC are included in calculations of the company's financial stability and take all legal and regulatory measures to ensure that SENELEC regularly satisfies its tax and customs obligations. SENELEC will provide a statement of its tax and customs obligations, at a minimum on a quarterly basis, to the Minister of Finance; 
- $\quad$ Return the GTI power station to service while maintaining existing contractual stipulations regarding the guarantee by the state without other financial risk for the government;

- Mobilize private bank loans in the amount of CFAF 36 billion against payment in two tranches in 2011 and 2012 of the balance of the 2008 compensation to SENELEC by the government for the freezing of electricity tariffs. However, the net debt by SENELEC to the government resulting from the agreement to offset mutual claims, as well as all new debt of SENELEC vis-à-vis the government accumulated in 2010, will be deducted from the bank loans before the proceeds are made available to SENELEC;

- $\quad$ Finalize the terms and conditions for restructuring SENELEC's short-term debt to reduce the stock of arrears and the high cost of debt servicing; and

- Undertake an assessment of the fuel supply and human resource management functions.

25. The government is committed to implement a detailed action plan in line with regulations to meet the conditions of the second tranches of budget support from the World Bank and AFD relating to the restructuring of the sector, currently planned for 2011. In cooperation with the World Bank and AFD, the government intends to unbundle SENELEC's activities (production, transport, and distribution) in the medium term and to finalize its strategy for private participation.

26. To improve tracking by the authorities, the government has transferred the management of the amounts collected by SAR as FSIPP (Fonds de Sécurisation des Importations en Produits Pétroliers) assessments from the Treasury to the DGID. Despite some implementation delays, the DGID has begun collecting FSIPP payments made by SAR clients. It deposits the payments in the account of the General Treasurer opened at the BCEAO that is dedicated to FSIPP. On the 25 th of each month, the General Treasurer gives the BCEAO an order for a transfer to a SAR account opened at ECOBANK to allow the bank to be reimbursed. The government is committed to include all subsidies and transfers to SAR, including FSIPP payments collected by the DGID and all strategic investments in the oil sector, in the first supplementary budget for 2010 .

\section{Financial sector reforms}

27. There were some cash flow difficulties in the financial sector in late $\mathbf{2 0 0 8}$ linked to domestic factors as well as the global crisis. Nonperforming loans have stabilized, but several banks still fail to meet certain prudential regulations. Against this backdrop, the authorities are determined to make every effort to maintain stability in the financial sector. The government action plan lists measures taken to date and identifies priorities for the coming year. In particular, the authorities undertake to: 
(a) Continue to monitor the banks, including analyzing bank assets and liabilities, the nature and concentration of financing sources, liquidity relationships with parent countries or institutions in the same group abroad, and overall liquidity. It will also intensify discussions with the WAEMU authorities and supervisors of foreign institutions with a presence in Senegal;

(b) Focus on macrofinancial risks arising from financing links between the government, private sector, and financial system. Banks will be subject to regular stress tests;

(c) Step up consultations with the government and banks to anticipate financing and cash requirements of the government and private sector;

(d) In consultation with the central bank, closely monitor the entry into force of the new minimum capital rules by end-2010. In this connection, a working group made up of officials from the BCEAO and a representative of the Ministry of Finance has been created in each WAEMU country at the initiative of the BCEAO and the Banking Commission to review the position of credit institutions likely to encounter difficulties in complying with the new rules and proposing targeted measures;

(e) Further strengthen the capacity and resources of the directorate in charge of regulation and supervision of microfinance institutions within the Ministry of Economy and Finance.

28. The government aims to improve the institutional, legal, and operational environment for private sector financing to enhance household access to credit and expand financial intermediation. The following initiatives will be taken:

(a) implementation of an action plan to increase the rate of bank penetration to 20 percent by end-2012 through, inter alia, government promotion of noncash means of payments;

(b) establishment of a legal framework that supports the creation of credit information bureaus;

(c) finalization of legislation governing financial leasing to promote financing of start-ups and growth of innovative small and medium-sized enterprises.

\section{Developing the private sector and improving governance}

\section{Accelerating growth requires rapid implementation of several key reforms.}

The government is determined to accelerate the momentum to improve the business environment and reach international standards. Senegal's position in the World Bank's "Doing Business" indicators has fallen. Key areas for improvement include strengthening 
contract enforcement, reducing delays in transferring property rights, modernizing labor legislation, simplifying customs procedures, and improving access to finance. The government specifically commits to achieve the following as soon as possible:

(a) improve the efficiency of the commercial justice system by providing courts with the resources required for the expeditious handling of economic disputes and implementing administrative measures already identified;

(b) reduce delays in the transfer of property rights as specified in office memoranda issued in 2008 and adopt and effectively implement the Prime Minister's circular regarding reductions in the time it takes to issue construction permits and hook up water, sewage, electricity, and telephone services;

(c) extend possibilities for renewing fixed-term labor contracts;

(d) implement the action plan that came out of the second national forum on credit, which took place on March 16 and 17, 2010, according to a timetable to be agreed.

\section{The government is also introducing various measures designed to improve governance as follows:}

(a) finalizing legislation on government property and real estate: (i) the law on real estate ownership and its implementation decree; (ii) updating of the price schedules for government-owned property;

(b) strengthening of the Audit Court by providing it with the appropriate level of resources and promoting the training of judges with the help of donors;

(c) introducing measures to guarantee the availability of appropriate resources for the procurement regulatory agency (ARMP); and

(d) introducing measures to ensure that the National Commission to Enhance Transparency and Combat Corruption and Extortion has sufficient resources to perform its work.

\section{Program Monitoring}

\section{Quantitative assessment criteria for end-June $\mathbf{2 0 1 0}$ and quantitative} indicators for end-September 2010 have been set to monitor program implementation in 2010 (see Table 1 below). The government and IMF staff also agreed on the structural benchmarks listed in Table 2 below. The sixth review under the PSI is expected to be completed by end-October 2010. The PSI expires on November 1. The government is resolved to continue to work closely with the IMF. The terms and conditions for the continuation of this collaboration will be examined during the next mission. 
Table 1 of MEFP. Quantitative Assessment Criteria and Indicative Targets for 2010 1/

(CFAF billions, unless otherwise specified)

\begin{tabular}{|c|c|c|c|}
\hline & \multicolumn{2}{|c|}{ June 30, 2010} & \multirow[t]{2}{*}{$\begin{array}{c}\text { September 30, } \\
2010\end{array}$} \\
\hline & Existing & & \\
\hline \multicolumn{4}{|l|}{ Assessment criteria } \\
\hline Floor on the basic fiscal balance $2 /$ & -59 & -49 & -73 \\
\hline \multicolumn{4}{|l|}{ Ceiling on the contracting or guaranteeing of new } \\
\hline $\begin{array}{l}\text { nonconcessional external debt by the government } 3 / 4 / 5 / \\
\text { Ceiling on spending undertaken outside normal and simplified }\end{array}$ & 0 & 0 & 0 \\
\hline $\begin{array}{l}\text { procedures } 4 / \\
\text { Ceiling on government external payment arrears (stock) } 4 /\end{array}$ & $\begin{array}{l}0 \\
0\end{array}$ & $\begin{array}{l}0 \\
0\end{array}$ & $\begin{array}{l}0 \\
0\end{array}$ \\
\hline \multicolumn{4}{|l|}{ Ceiling on the amount of the float (depenses liquidees } \\
\hline non payees par le Tresor) 6/ & 45 & 45 & 45 \\
\hline \multicolumn{4}{|l|}{ Indicative target } \\
\hline $\begin{array}{l}\text { Quarterly ceiling on the share of the value of public sector } \\
\text { contracts signed by single tender (percent) }\end{array}$ & 20 & 20 & 20 \\
\hline
\end{tabular}

1/ Indicative targets for September 2010, except for the assessment criteria monitored on a continuous basis.

2/ Defined as total revenue minus total expenditure and net lending, excluding externally financed capital expenditure,

on-lending, HIPC and MDRI spending, and payment of extrabudgetary spending and agency debt identified in the

July 2009 audit and included in the first supplementary budget of 2010. Cumulative since the beginning of the year.

Total revenue excludes privatization receipts and sales of mobile telephone licenses.

3/ Excluding government or government-guaranteed CFAF borrowing from financial institutions within the WAEMU and external loans contracted by the airport project company (AIDB) to finance the construction of the new Dakar Airport.

4/ Monitored on a continuous basis.

5/ Cumulative since approval of fourth PSI review.

6/ Defined as all expenditure for which a bill has been received and recognized (dépense liquidée) but not yet paid by the Treasury. 
Table 2 of the MEFP: Structural Benchmarks, May-October 2010

\begin{tabular}{|c|c|c|c|}
\hline Measures & $\begin{array}{l}\text { MEFP } \\
\S\end{array}$ & $\begin{array}{l}\text { Implementation } \\
\text { Date }\end{array}$ & Macroeconomic Rationale \\
\hline $\begin{array}{l}\text { 1. Submit a supplementary budget for } 2010 \text { to } \\
\text { Parliament, based on the macroeconomic } \\
\text { framework agreed with IMF staff, which } \\
\text { includes specific line items authorizing the } \\
\text { Ministry of Finance to settle in } 2010 \text { the } \\
\text { extrabudgetary arrears of ministries and public } \\
\text { institutions and agencies and part of the public } \\
\text { institution and agency debt identified in the July } \\
2009 \text { audit and not yet budgeted for. Any debt } \\
\text { settlement not requiring a budget allocation will } \\
\text { be presented in an annex to the supplementary } \\
\text { budget. This process is accompanied by a } \\
\text { press release explaining the settlement terms } \\
\text { and conditions. }\end{array}$ & 5,9 & May 15,2010 & $\begin{array}{l}\text { Complete the normalization of } \\
\text { financial relations with the private } \\
\text { sector. }\end{array}$ \\
\hline $\begin{array}{l}\text { 2. Improve SIGFIP by including payroll } \\
\text { expenditure and implementing the SIGFIP- } \\
\text { ASTER interface. }\end{array}$ & 18 & July 31, 2010 & $\begin{array}{l}\text { Strengthen the monitoring of } \\
\text { budget execution. }\end{array}$ \\
\hline $\begin{array}{l}\text { 3. Conduct a census of all accounts of general } \\
\text { government and public institutions, including } \\
\text { the number, name of the holder, and the } \\
\text { balances as of December } 31,2008 \text { and } 2009 \\
\text { for each account, with a view to adopting a } \\
\text { strategy to establish a single Treasury account. }\end{array}$ & 20 & July 31, 2010 & $\begin{array}{l}\text { Improve the management of cash } \\
\text { flows, as well as the transparency } \\
\text { of operations. }\end{array}$ \\
\hline $\begin{array}{l}\text { 4. Complete payment of at least } 50 \text { percent of } \\
\text { extrabudgetary arrears and public institution } \\
\text { and agency debt identified in the July } 2009 \\
\text { audit. }\end{array}$ & 9 & $\begin{array}{l}\text { September 30, } \\
2010\end{array}$ & $\begin{array}{l}\text { Complete the normalization of } \\
\text { financial relations with the private } \\
\text { sector and improve the business } \\
\text { climate. }\end{array}$ \\
\hline $\begin{array}{l}\text { 5. Publish a press release reporting on the } \\
\text { results of the internal audit, summarizing } \\
\text { progress made to clear the extrabudgetary } \\
\text { commitments and public institution and agency } \\
\text { debt, and detailing the steps taken to complete } \\
\text { this process. }\end{array}$ & 10 & $\begin{array}{l}\text { September } 30 \\
2010\end{array}$ & $\begin{array}{l}\text { Complete the normalization of } \\
\text { financial relations with the private } \\
\text { sector and improve the business } \\
\text { climate. }\end{array}$ \\
\hline $\begin{array}{l}\text { 6. Compile the general tax code and all } \\
\text { legislation governing domestic taxation in a } \\
\text { single document. }\end{array}$ & 23 & October 15, 2010 & Further simplify the tax system. \\
\hline
\end{tabular}




\title{
ATtaChment II \\ TeChNiCAL MEMORANDUM OF UNDERSTANDING
}

\author{
Dakar, May 7, 2010
}

1. This technical memorandum of understanding (TMU) defines the quantitative assessment (performance) criteria, indicative targets, and structural benchmarks on the basis of which the implementation of the Fund-supported program under the Policy Support Instrument (PSI) will be monitored in 2010. The quantitative program targets will also serve as performance criteria under the Exogenous Shocks Facility (ESF). The TMU also establishes the terms and time frame for transmitting the data that will enable Fund staff to monitor program implementation

\section{PROGRAM CONDITIONALITY}

2. The quantitative assessment criteria for June 30, 2010 and the quantitative indicative targets for September 30, 2010 are shown in Table 1. The prior actions and structural benchmarks established under the program are presented in Table 2.

\section{Definitions, Adjusters, ANd DATA Reporting}

\section{A. The Government}

3. Unless otherwise specified below, the government is defined as the central administration of the Republic of Senegal and does not include any local administration, the central bank, or any government-owned entity with a separate legal personality (e.g., public universities and hospitals).

\section{B. Basic Fiscal Balance (Program Definition)}

\section{Definition}

4. The basic fiscal balance (program definition) is the difference between the government's budgetary revenue and total expenditure and net lending, excluding externally financed capital expenditure (financed by donors), drawings on on-lent loans (except on-lent loans to the energy sector financed through donor budgetary assistance), expenditure funded with HIPC- and MDRI-related resources, and expenditure related to the settlement of agency debt and extrabudgetary arrears identified in the July 2009 audit and included in the first supplementary budget for 2010. Budgetary revenue excludes privatization receipts and sales of mobile telephone licenses or other government assets. Government expenditure is defined on the basis of payment orders accepted by the Treasury (dépenses ordonnancées prises en 
charge par le Trésor). This assessment/performance criterion is set as a floor on the cumulative basic fiscal balance since the beginning of the year.

\section{Example}

5. The floor for the basic balance (program definition) as at June 30, 2010 is CFAF 49 billion. It is calculated as the difference between budgetary revenue (CFAF 614 billion) and total expenditure and net lending (CFAF 834 billion), excluding externally financed capital expenditure (CFAF 137 billion), drawings on on-lent loans (CFAF 5 billion), and expenditure funded with HIPC-and MDRI-related resources (CFAF 30 billion).

\section{Reporting requirements}

6. During the program period, the authorities will report provisional data on the basic fiscal balance (program definition) and its components monthly to Fund staff with a lag of no more than 30 days. Data on revenues and expenditure that are included in the calculation of the basic fiscal balance, and on expenditure financed with HIPC- and MDRI- related resources, will be drawn from preliminary Treasury account balances. Final data will be provided as soon as the final balances of the Treasury accounts are available, but not later than two months after the reporting of the provisional data.

\section{Adjuster for the Basic Fiscal Balance}

\section{Definition}

7. The floor on the basic fiscal balance does not take into account any privatization receipts. However, if privatization receipts occur in 2010, spending will be increased and the floor on the basic fiscal balance correspondingly raised by up to CFAF 35 billion.

\section{Example}

8. If CFAF 100 billion in privatization receipts were to become available in 2010, spending would be carried out in line with the initial budget and would be raised by CFAF 35 billion, from CFAF 1668 billion to CFAF 1703 billion. The floor on the basic fiscal balance would be lowered by CFAF 35 billion, from -CFAF 97 billion to -CFAF 132 billion.

\section{Budgetary Float}

\section{Definition}

9. The budgetary float (instances de paiement) is defined as the outstanding stock of government expenditure for which bills have been received and validated but not yet paid by the Treasury (the difference between dépenses liquidées and dépenses payées). The 
assessment/performance criterion is set as a ceiling on the budgetary float, monitored at the end of the quarter.

\section{Reporting requirements}

10. The authorities will transmit to Fund staff on a weekly basis (i.e., at the end of each week), and at the end of each month, a table from the expenditure tracking system (SIGFIP) showing all committed expenditures (dépenses engagées), all certified expenditures that have not yet been cleared for payment (dépenses liquidées non encore ordonnancées), all payment orders (dépenses ordonnancées), all payment orders accepted by the Treasury (dépenses prises en charge par le Trésor), and all payments made by the Treasury (dépenses payées). The SIGFIP table will exclude delegations for regions and embassies. The SIGFIP table will also list any payments that do not have a cash impact on the Treasury accounts.

\section{E. Spending Undertaken Outside Simplified and Normal Procedures}

11. This assessment/performance criterion is applied on a continuous basis to any procedure other than the normal and simplified procedures to execute spending. It excludes only spending undertaken on the basis of an advance decree in cases of absolute urgency and need in the national interest, in application of Article 12 of the Organic Budget Law. Such spending requires the signatures of the President of the Republic and Prime Minister. This criterion has been monitored from the time of the second PSI review.

12. The authorities will report any such procedure, together with the SIGFIP table defined in paragraph 8 , to Fund staff on a monthly basis with a maximum delay of 30 days.

\section{F. Government External Payments Arrears}

\section{Definition}

13. External payments arrears are defined as the sum of payments owed and not paid on the external debt contracted or guaranteed by the government. The definition of external debt given in paragraph 13 is applicable here. The assessment (performance) criterion on external payments arrears will be monitored on a continuous basis.

\section{Reporting requirements}

14. The authorities will promptly report any accumulation of external payments arrears to Fund staff. 


\section{G. Contracting or Guaranteeing of New Nonconcessional External Debt by the Government}

\section{Definition}

15. This assessment/performance criterion applies not only to debt as defined in Point No. 9 of the Guidelines on Performance Criteria with Respect to Foreign Debt (Executive Board Decision No. 6230-(79/140), last amended by Executive Board Decision No. 12274(00/85), adopted August 24, 2000, but also to commitments contracted or guaranteed by the government for which funds have not been received. ${ }^{1}$ The criterion does not apply to:

(i) CFAF debt contracted or guaranteed by the government with WAEMU residents;

(ii) CFAF debt initially contracted or guaranteed by the government with WAEMU residents subsequently acquired by nonresidents;

(iii) CFAF government or government-guaranteed debt where the agreement is between the government and a WAEMU resident entity and there is no ensuing contractual obligation between the government and a nonresident entity, regardless of whether the resident WAEMU entity resells the debt to a nonresident;

(iv) debt rescheduling transactions of debt existing at the time of the approval of the PSI; and

(v) external debt contracted by the airport project company (AIDB) to finance construction of the new Dakar Airport.

16. This criterion is measured on a cumulative basis from the time of approval of the fourth program review and applies continuously. No adjuster will be applied to this criterion.

17. For purposes of this assessment/performance criterion, government is understood to include the government as defined in paragraph 3 above, as well as public institutions of an industrial and commercial nature (EPIC), public administrative institutions (EPA), public institutions of a scientific and technical nature, public institutions of a professional nature, public health institutions, local administrations, public enterprises, and government-owned or controlled independent companies (sociétés nationales) (i.e., public enterprises with financial

\footnotetext{
${ }^{1}$ The following reference to the IMF's external website links to a tool that allows the calculation of the grant element for a broad range of financing packages http://www.imf.org/external/np/pdr/conc/calculator/default.aspx
} 
autonomy where the government holds at least 50 percent of the capital), and government agencies.

18. Any external debt of which the net present value, calculated with the reference interest rates mentioned hereafter, is greater than 65 percent of the nominal value (grant element of less than 35 percent) is considered nonconcessional, with the exception of IMF lending. For debt with a maturity of more than 15 years, the average of the ten-year commercial interest reference rates (CIRRs) published by the OECD is used to calculate the grant element. The average of the six-month CIRRs is used for debt with shorter maturities. For loans in foreign currencies for which the OECD does not calculate a CIRR, calculation of the grant element should be based on the CIRR in SDRs.

\section{Reporting requirements}

19. The government will report any new external borrowing and its terms to Fund staff as soon as external debt is contracted or guaranteed by the government.

\section{H. Public Sector Contracts Signed by Single Tender}

\section{Definition}

20. Public sector contracts are administrative contracts, drawn up and entered into by the government or any entity subject to the procurement code, for the procurement of supplies, delivery of services, or execution of work. Public sector contracts are considered "singletender" contracts when the contracting agent signs the contract with the chosen contractor without competitive tender. The quarterly indicative target will apply to public sector contracts examined by the Central Public Procurement Directorate (DCMP).

\section{Reporting requirements}

21. The government will report quarterly to Fund staff, with a lag of no more than one month from the end of the observation period, the total value of contracts signed by all ministries and agencies and the total value of all single-tender contracts signed by these ministries and agencies.

\section{AdDitional Information for Program Monitoring}

22. The authorities will transmit the following to Fund staff, with the maximum time lags indicated:

(a) Effective immediately: any decision, circular, edict, decree, ordinance, or law having economic or financial implications for the current program. 
(b) With a maximum lag of 30 days, preliminary data on:

- Tax receipts and tax and customs assessments by categories, accompanied by the corresponding revenue;

- $\quad$ The monthly amount of expenditures committed, certified, and for which payment orders have been issued;

- $\quad$ The quarterly report of the Debt and Investments Directorate (DDI) on the execution of investment programs;

- $\quad$ The monthly preliminary government financial operations table (TOFE), based on the Treasury accounts;

- $\quad$ The provisional balance of the Treasury accounts; and

- $\quad$ Reconciliation tables between the SIGFIP table and the consolidated Treasury accounts, between the consolidated Treasury accounts and the TOFE for "budgetary revenues," between the consolidated Treasury accounts and the TOFE for "total expenditure and net lending," and between the TOFE and the net government position (NGP), on a quarterly basis.

(c) Final data will be provided as soon as the final balances of the Treasury accounts are available, but not later than one month after the reporting of provisional data.

23. During the program period, the authorities will transmit provisional data on current nonwage noninterest expenditures and domestically financed capital expenditures executed through cash advances to Fund staff on a monthly basis with a lag of no more than 30 days. The data will be drawn from preliminary consolidated Treasury account balances. Final data will be provided as soon as the final balances of the Treasury accounts are available, but no more than one month after the reporting of provisional data .

24. The government will transmit to Fund staff :

- $\quad$ The monthly balance sheet of the central bank, with a maximum lag of one month;

- $\quad$ The consolidated balance sheet of banks with a maximum lag of two months;

- $\quad$ The monetary survey, on a quarterly basis, with a maximum lag of two months;

- The lending and deposit interest rates of commercial banks, on a monthly basis; and 
- $\quad$ Prudential supervision and financial soundness indicators for bank financial institutions, as reported in the Table entitled Situation des Etablissements de Crédit vis-à-vis du Dispositif Prudentiel (Survey of Credit Institution Compliance with the Prudential Framework), on a quarterly basis.

25. The government will update monthly on the website used for this purpose the amount of airport tax - redevance de développement des infrastructures aéroportuaires (RDIA) collected, deposited in the escrow account, and used for the repayment of the loan financing the construction of the new airport. 
INTERNATIONAL MONETARY FUND

AND

INTERNATIONAL DEVELOPMENT ASSOCIATION

SENEGAL

Joint IMF/IDA Debt Sustainability Analysis

Prepared by the Staffs of the International Monetary Fund and the

International Development Association

Approved by Roger Nord and Thomas Dorsey (IMF)

and Sudhir Shetty and Sudarshan Gooptu (World Bank)

May 7, 2010

Senegal remains at a low risk of debt distress. Under the baseline scenario and various shock scenarios, all the debt burden indicators remain well below their indicative policy-dependent thresholds. External debt is vulnerable to a permanent deterioration in borrowing terms, which highlights the need for prudent debt management especially as the authorities seek greater access to external resources on nonconcessional terms. While the inclusion of domestic debt does not alter the overall assessment of Senegal's debt vulnerabilities, it raises the debt burden indicators (under the baseline and stress tests), suggesting the need for fiscal consolidation once the impact of the crisis subsides.

\section{BACKGROUND}

1. Most of Senegal's external debt is concessional. More than 60 percent of end-2009 external debt was owed to multilateral institutions (especially the World Bank and AfDB). Major bilateral creditors include France, Kuwait, Spain, China and India.

2. In December 2009, Senegal issued its first Euro Bond. The US\$200 million bond has a maturity of 5 years, and a coupon of 8.75 percent, but was priced to yield 9.25 percent. The proceeds of the issuance will finance the Dakar-Diamniadio toll road.

3. Domestic public debt is low. At end 2009, domestic debt reached 8 percent of GDP, or one-fourth of total debt. ${ }^{1}$ This debt is denominated in local currency and mostly held by WAEMU banks. In 2009, net domestic debt issuance reached almost 3 percent of GDP.

\footnotetext{
${ }^{1}$ Domestic debt includes debt issued in the WAEMU financial market.
} 
4. Private sector exposure also appears relatively limited. Private external debt was estimated at 20 percent of GDP at end-2009, limiting concerns about potential fiscal contingent liabilities stemming from private debt.

\section{UNDERLYING ASSUMPTIONS}

5. The macroeconomic framework rests on the implementation of sound macroeconomic and structural policies (Box 1).

- $\quad$ Growth is projected to accelerate over the next few years, as the effects of the international economic and financial crisis dissipate and the authorities continue their structural reforms aimed at raising growth.

- $\quad$ Over the long run, real GDP growth is projected to exceed 5 percent. Between 1995 (after the devaluation) and 2007 (before the food, fuel and financial crisis), real GDP growth averaged about $4 \frac{1}{2}$ percent. The long-run projections assume that Senegal reduces constraints to growth through continued structural reforms, including in the business climate, the energy and financial sectors, as well as labor markets. The projections also assume successful implementation of its infrastructure program (including road, port, and airport).

- $\quad$ FDI (net) is expected to remain relatively low over the short run, in part owing to the financial crisis. It is expected to pick up, as economic prospects improve and uncertainty is reduced, to average about 3 percent of GDP in the long term.

- $\quad$ The primary fiscal deficit is expected to decline over the medium term, as the impact of the crisis subsides. Fiscal consolidation is expected to continue in order to safeguard debt sustainability. Most of Senegal's public financing needs are projected to be filled through external concessional borrowing.

6. Compared to the previous DSA, macroeconomic assumptions have been revised to reflect more updated information regarding the impact of the crisis. ${ }^{2}$ GDP growth has been revised down from 3.1 percent in 2009 to 1.5 percent, while long-term real GDP growth remains unchanged. The current account deficit (excluding interest payments) in 2009 has been revised down by about 2 percentage points, reflecting a sharper-than-expected contraction in imports and stronger-than-expected remittances.

\footnotetext{
${ }^{2}$ In the previous DSA, completed in June 2009 (Country Report 09/205). Senegal was classified as a low risk of debt distress.
} 


\section{Box 1. Macroeconomic Assumptions for 2010-30}

Real GDP growth: Real GDP growth is expected to pick up once the effect of the global economic and financial crisis subsides. In particular, growth is expected to increase from 1.5 percent in 2009 , to an average of 3.8 percent over $2010-11,4.8$ percent over $2012-15$, and over $5 \frac{1}{4}$ percent over the long term.

Inflation: Inflation, as measured by the GDP deflator (in US. dollar), is expected to increase as excess capacity is reduced. Inflation is expected to stabilize at around 2 percent.

Current account deficit (excluding interest payments): the current account deficit is expected to deteriorate slightly over the medium term as uncertainty abates and economic prospects improve. The current account deficit excluding interest payments is expected to stabilize at around 8.5 percent by the end of the projection period, as the growth of exports overtakes that of imports. Remittances are expected to grow slowly over the medium term after a strongerthan-expected performance in 2009 (despite the crisis).

Fiscal deficit: after a primary deficit of 4.4 percent of GDP in 2009, modest primary fiscal deficits averaging 3.4 percent of GDP are expected in 2010 and 2011, reflecting a reduction in the need for countercyclical fiscal policy. Thereafter, the primary deficit gradually declines as public expenditure management - a reform focus under the program supported by the IMF's Policy Support Instrument and the Bank's budget support operations (PFSC and PRSCs) continues to be improved, and further efficiency gains are being made in tax administration.

Aid flows (grants and concessional loans): access to concessional resources is expected to decline as Senegal's development improves. Grants are expected to decline gradually from 2.7 percent of GDP in 2010 to 2.1 percent of GDP by the end of the projection period, while concessional loans are expected to decline from 1.9 percent of GDP to 1.7 percent of GDP over the same period.

Public domestic borrowing: as the WAEMU debt markets become more liquid and efficient, Senegal is expected to rely increasingly on domestic financing. Senegal's domestic borrowing is expected to be less than one-third of the total public financing needs and claims on the government are expected to be largely held by commercial banks.

\section{EXTERNAL DSA}

\section{External PPG debt burden indicators under the baseline scenario remain well} below their policy-dependent thresholds (Figure 1, Table 1). ${ }^{3}$ Workers' remittances represent a reliable and large source of foreign exchange for Senegal, accounting for around 10 percent of GDP and more than 40 percent of exports over the projection period. As such, they are explicitly considered in this DSA (Figure 2). ${ }^{4}$ The modified debt burden indicators-

\footnotetext{
${ }^{3}$ The indicative external debt burden thresholds for Senegal are shown in Figure 1. They are based on Senegal's classification as a "medium" performer given its (three-year average) score of 3.67 on the World Bank's Country Policy and Institutional Assessment index (CPIA). The CPIA measures the quality of policies and institutions; weak performers score below 3.25 , strong performers above 3.75 .

${ }^{4}$ For a detailed discussion of the treatment of remittances in LIC DSAs, please see "Staff Guidance Note on the Application of the Joint Fund-Bank Debt Sustainability Framework for Low-Income Countries, " SM/10/16.
} 
the PV of external debt and external debt service as ratios to the sum of exports and gross remittances, and to the sum of gross domestic product and gross remittances, respectivelyare significantly lower than the updated DSF thresholds (10 percent lower than the usual DSF thresholds).

8. Stress tests do not reveal serious vulnerabilities for external public debt, as the various indicators remain below the thresholds (Figure 2, Table 2). The most common extreme stress test is a general deterioration in the terms of external borrowing, i.e., an increase by 2 percentage points in the interest rate on new external public borrowing. Despite this adverse shock, the debt burden indicators remain well below their thresholds.

\section{Public DSA}

9. Indicators of overall public debt (external plus domestic debt) and debt service follow a similar pattern as those for external public debt alone (Table 3, Figure 3 ). While more elevated than under the external DSA, the public debt burden indicators do not suggest increased concerns for debt sustainability.

10. Public debt sustainability hinges on containing the fiscal deficit in the medium and long term. If the fiscal balance were to remain at its 2010 level, the debt burden indicators would appear to be on an upward trend, suggesting that the debt situation is unsustainable, highlighting the importance of an exit strategy for fiscal stimulus.

11. The public debt position is also vulnerable to shocks to real GDP growth. Under a scenario with permanently lower real growth, the PV of debt-to-GDP stabilizes at close to 50 percent, which is about 10 percentage points above the baseline. This highlights the need for the authorities to continue pursuing their goal of raising potential output growth.

\section{Conclusion}

12. Senegal's external debt burden is subject to a low risk of debt distress. The sustainability of Senegal's external PPG debt seems to be vulnerable to a permanent deterioration in its borrowing terms. This highlights the need for prudent debt management by Senegal, especially as it seeks to gain greater access to external resources on nonconcessional terms. Adding domestic debt, while raising the debt burden indicators, does not change the overall assessment of Senegal's debt vulnerabilities, but highlights the need for fiscal consolidation once the impact of the crisis subsides. 


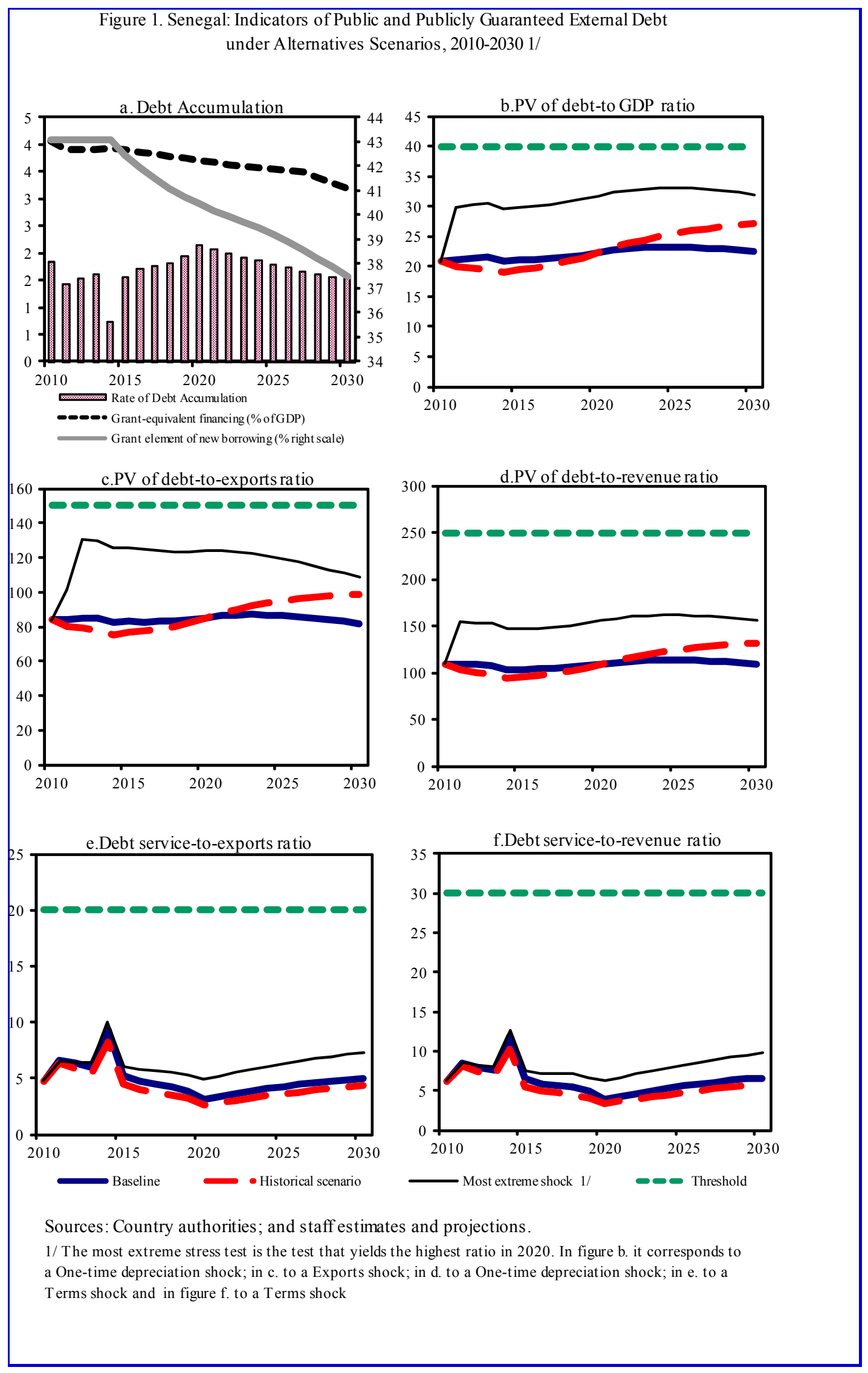


Table 1.: External Debt Sustainability Framework, Baseline Scenario, 2007-2030 1/ (In percent of GDP, unless otherwise indicated)

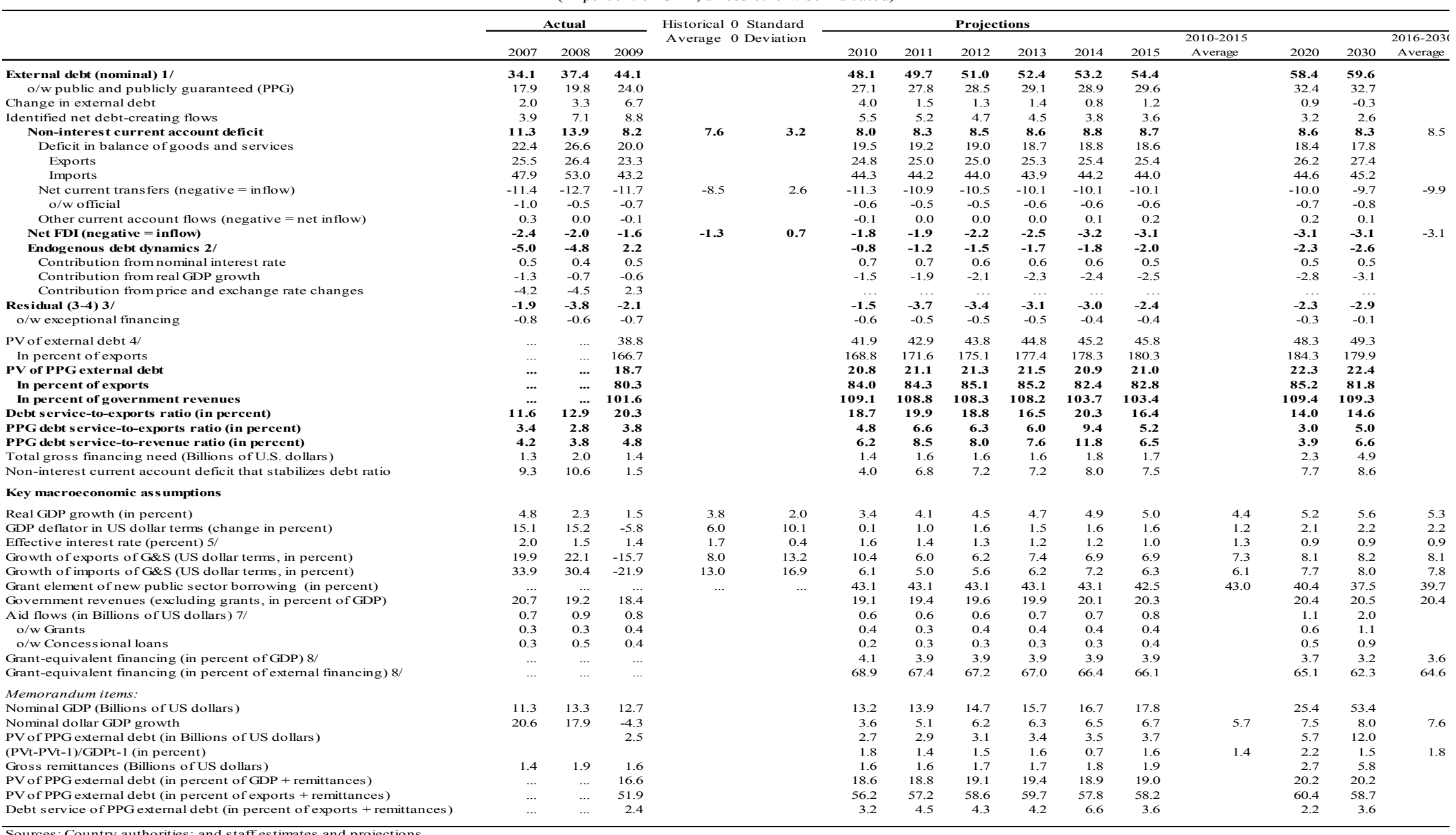

Source: Countiy authorities; and staff estimates and projections.

1/ Includes both public and private sector external debt.

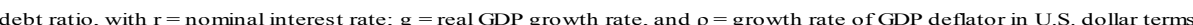

3/ Includes exceptional financing (i.e., changes in arrears and debt relief); cho

4/ Assumes that PV of private sector debt is equivalent to its face value.
$5 /$ Current-year interest payments divided by previous period debt stock.

$6 /$ Historical averages and standard deviations are generally derived over the past 10 years, subject to data availability.

8/ Grant-equivalent financing includes grants provided directly to the government and through new borrowing (difference between the face value and the PV of new debt). 


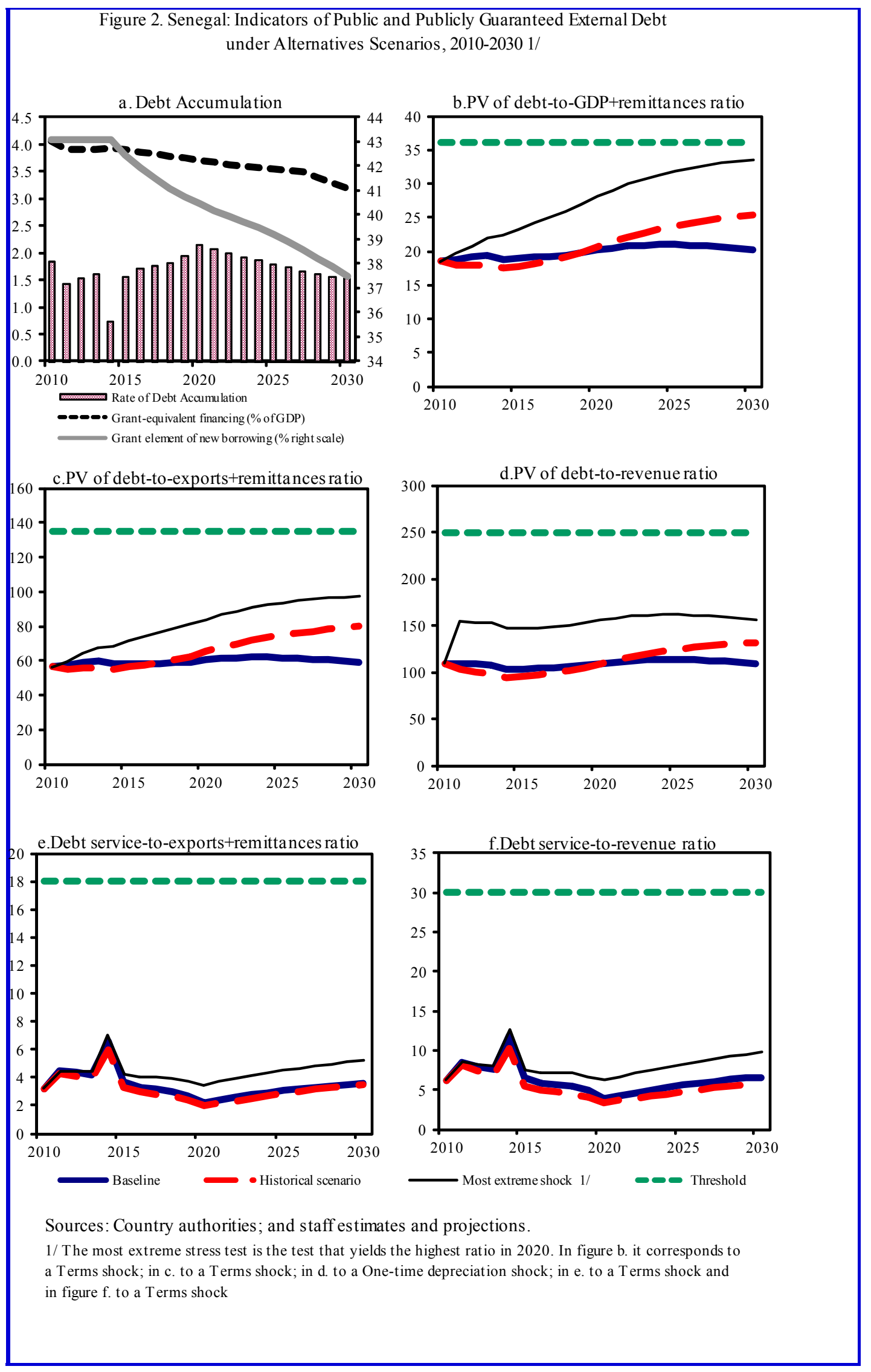


Table 2.Senegal: Sensitivity Analysis for Key Indicators of Public and Publicly Guaranteed External Debt, 2010-2030 (In percent)

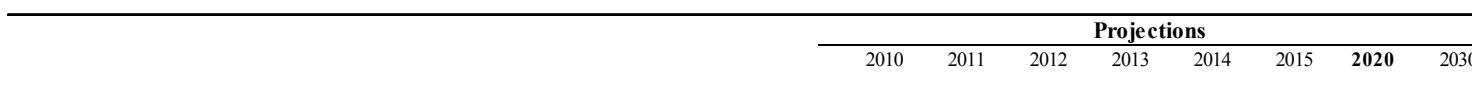

PV of debt-to-GDP+re mittances ratio

Baseline

19

A. Alternative Scenarios

A1. Key variables at their historical averages in 2010-2030 1/

A2. New public sector loans on less favorable terms in 2010-20302

$\begin{array}{llllllll}19 & 18 & 18 & 18 & 17 & 18 & \mathbf{2 1} & 25 \\ 19 & 20 & 21 & 22 & 22 & 23 & \mathbf{2 8} & 34\end{array}$

B. Bound Tests

B1. Real GDP growth at historical average minus one standard deviation in 2011-2012

B2. Export value growth at historical average minus one standard deviation in 2011-2012 3/

B3. US dollar GDP deflator at historical average minus one standard deviation in 2011-2012

B4. Net non-debt creating flows at historical average minus one standard deviation in 2011-2012 4/

B5. Combination of B1-B4 using one-half standard deviation shocks

B6. One-time 30 percent nominal depreciation relative to the baseline in $20115 /$

\section{PV of debt-to-exports + re mittances ratio}

Baseline

A. Alternative Scenarios

A1. Key variables at their historical averages in 2010-2030 1/

A2. New public sector loans on less favorable terms in 2010-20302

B. Bound Tests

B1. Real GDP growth at historical average minus one standard deviation in 2011-2012 B2. Export value growth at historical average minus one standard deviation in 2011-2012 3/

B3. US dollar GDP deflator at historical average minus one standard deviation in 2011-2012

B4. Net non-debt creating flows at historical average minus one standard deviation in 2011-2012 4/

B5. Combination of B1-B4 using one-half standard deviation shocks

B6. One-time 30 percent nominal depreciation relative to the baseline in 20115

$\begin{array}{llllllll}56 & 57 & 59 & 60 & 58 & 58 & \mathbf{6 0} & 59 \\ & & & & & & & \\ 56 & 55 & 56 & 56 & 55 & 56 & \mathbf{6 5} & 80 \\ 56 & 60 & 64 & 68 & 68 & 71 & \mathbf{8 4} & 97 \\ & & & & & & & \\ & & & & & & & \\ 56 & 57 & 58 & 60 & 58 & 58 & \mathbf{6 0} & 59 \\ 56 & 66 & 83 & 85 & 82 & 82 & \mathbf{8 2} & 73 \\ 56 & 57 & 58 & 60 & 58 & 58 & \mathbf{6 0} & 59 \\ 56 & 80 & 93 & 81 & 78 & 78 & \mathbf{7 6} & 64 \\ 56 & 78 & 96 & 86 & 83 & 82 & \mathbf{8 1} & 68 \\ 56 & 57 & 58 & 60 & 58 & 58 & \mathbf{6 0} & 59\end{array}$

PV of debt-to-revenue ratio

Baseline

109

109

108

108

109

109

A. Alternative Scenarios

A1. Key variables at their historical averages in 2010-2030 1/

A2. New public sector loans on less favorable terms in 2010-2030 2

109

109
109

114

100

98

95

$95 \quad \mathbf{1 0}$

B. Bound Tests

B1. Real GDP growth at historical average minus one standard deviation in 2011-2012

B2. Export value growth at historical average minus one standard deviation in 2011-2012 3/

B3. US dollar GDP deflator at historical average minus one standard deviation in 2011-2012

B4. Net non-debt creating flows at historical average minus one standard deviation in 2011-2012 4/

B5. Combination of B1-B4 using one-half standard deviation shocks

B6. One-time 30 percent nominal depreciation relative to the baseline in 2011 5/

$\begin{array}{llllllll}109 & 111 & 114 & 113 & 109 & 108 & \mathbf{1 1 5} & 115 \\ 109 & 117 & 133 & 132 & 126 & 125 & \mathbf{1 2 7} & 116 \\ 109 & 114 & 121 & 120 & 115 & 115 & \mathbf{1 2 2} & 122 \\ 109 & 129 & 148 & 146 & 140 & 138 & \mathbf{1 3 8} & 120 \\ 109 & 129 & 155 & 153 & 147 & 145 & \mathbf{1 4 4} & 124 \\ 109 & 154 & 154 & 153 & 147 & 147 & \mathbf{1 5 6} & 155\end{array}$


Table 2.Senegal: Sensitivity Analysis for Key Indicators of Public and Publicly Guaranteed External Debt, 2010-2030 (continued) (In percent)

Debt service-to-exports +remittances ratio

Baseline

A. Alternative Scenarios

A1. Key variables at their historical averages in 2010-2030 1/ A2. New public sector loans on less favorable terms in 2010-20302

\section{B. Bound Tests}

B1. Real GDP growth at historical average minus one standard deviation in 2011-2012 B2. Export value growth at historical average minus one standard deviation in 2011-2012 3/

B3. US dollar GDP deflator at historical average minus one standard deviation in 2011-2012 B4. Net non-debt creating flows at historical average minus one standard deviation in 2011-2012 4/

B5. Combination of B1-B4 using one-half standard deviation shocks

B6. One-time 30 percent nominal depreciation relative to the baseline in 20115 /

$\begin{array}{lllll}3 & 4 & 4 & 4 & 6 \\ 3 & 4 & 4 & 4 & 7\end{array}$

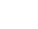

\section{Debt service-to-revenue ratio}

\section{Baselin}

A. Alternative Scenarios

A1. Key variables at their historical averages in 2010-2030 1/

A2. New public sector loans on less favorable terms in 2010-20302

\section{B. Bound Tests}

B1. Real GDP growth at historical average minus one standard deviation in 2011-2012

B2. Export value growth at historical average minus one standard deviation in 2011-2012 3 /

B3. US dollar GDP deflator at historical average minus one standard deviation in 2011-2012

B4. Net non-debt creating flows at historical average minus one standard deviation in 2011-2012 4

B5. Combination of B1-B4 using one-half standard deviation shocks

B6. One-time 30 percent nominal depreciation relative to the baseline in 20115 /

Memorandum item:

Grant element assumed on residual financing (i.e., financing required above baseline) 6/

$\begin{array}{lllllll}4 & 4 & 4 & 7 & 4 & \mathbf{2} & 4 \\ 5 & 5 & 5 & 8 & 5 & \mathbf{3} & 5 \\ 4 & 4 & 4 & 7 & 4 & \mathbf{2} & 4 \\ 5 & 5 & 5 & 7 & 4 & \mathbf{2} & 4 \\ 5 & 5 & 5 & 7 & 4 & \mathbf{3} & 4 \\ 4 & 4 & 4 & 7 & 4 & \mathbf{2} & 4\end{array}$

Sources: Country authorities; and staff estimates and projections.

1/ Variables include real GDP growth, growth of GDP deflator (in U.S. dollar terms), non-interest current account in percent of GDP, and non-debt creating flows.

2/ Assumes that the interest rate on new borrowing is by 2 percentage points higher than in the baseline., while grace and maturity periods are the same as in the baseline.

3/ Exports values are assumed to remain permanently at the lower level, but the current account as a share of GDP is assumed to return to its baseline level after the shock (implicitly assuming an offsetting adjustment in import levels).

4/ Includes official and private transfers and FDI

5/ Depreciation is defined as percentage decline in dollar/local currency rate, such that it never exceeds 100 percent.

6/ Applies to all stress scenarios except for A2 (less favorable financing) in which the terms on all new financing are as specified in footnote 2. 
Table 3.Senegal: Public Sector Debt Sustainability Framework, Baseline Scenario, 2007-2030 (In percent of GDP, unless otherwise indicated)

\begin{tabular}{|c|c|c|c|c|c|c|c|c|c|c|c|c|c|c|c|}
\hline & \multicolumn{3}{|c|}{ Actual } & \multirow[b]{2}{*}{ Average } & \multirow[b]{2}{*}{$\begin{array}{r}\text { Standard } \\
\text { Deviation } \\
\end{array}$} & \multicolumn{5}{|l|}{ Estimate } & \multicolumn{5}{|c|}{ Projections } \\
\hline & 2007 & 2008 & 2009 & & & 2010 & 2011 & 2012 & 2013 & 2014 & 2015 & $\begin{array}{l}2010-15 \\
\text { Average }\end{array}$ & 2020 & 2030 & $\begin{array}{l}\text { 2016-30 } \\
\text { Average }\end{array}$ \\
\hline $\begin{array}{l}\text { Public sector debt } 1 / \\
\text { o/w foreign-currency denominated }\end{array}$ & $\begin{array}{l}24.5 \\
17.9\end{array}$ & $\begin{array}{l}25.1 \\
19.8\end{array}$ & $\begin{array}{l}32.1 \\
24.0\end{array}$ & & & $\begin{array}{l}35.7 \\
27.1\end{array}$ & $\begin{array}{l}38.0 \\
27.8\end{array}$ & $\begin{array}{l}39.6 \\
28.5\end{array}$ & $\begin{array}{l}41.0 \\
29.1\end{array}$ & $\begin{array}{l}42.2 \\
28.9\end{array}$ & $\begin{array}{l}42.6 \\
29.6\end{array}$ & & $\begin{array}{l}46.3 \\
32.4\end{array}$ & $\begin{array}{l}45.8 \\
32.7\end{array}$ & \\
\hline Change in public sector debt & 1.5 & 0.6 & 7.0 & & & 3.6 & 2.3 & 1.5 & 1.4 & 1.2 & 0.4 & & 0.7 & -0.4 & \\
\hline Identified debt-creating flows & -0.4 & 3.3 & 3.1 & & & 4.3 & 2.1 & 1.5 & 1.4 & 1.2 & 1.1 & & 0.6 & -1.4 & \\
\hline Primary deficit & 3.3 & 4.1 & 4.4 & 2.0 & 2.4 & 3.6 & 3.3 & 2.9 & 2.9 & 2.9 & 2.9 & 3.1 & 2.8 & 1.0 & 2.4 \\
\hline Revenue and grants & 23.6 & 21.8 & 21.7 & & & 21.8 & 21.8 & 22.1 & 22.3 & 22.6 & 22.8 & & 22.8 & 22.5 & \\
\hline of which: grants & 2.9 & 2.6 & 3.4 & & & 2.7 & 2.5 & 2.5 & 2.4 & 2.4 & 2.4 & & 2.4 & 2.0 & \\
\hline Primary (noninterest) expenditure & 26.9 & 25.9 & 26.2 & & & 25.4 & 25.1 & 25.0 & 25.2 & 25.5 & 25.6 & & 25.6 & 23.5 & \\
\hline Automatic debt dynamics & -3.3 & -0.5 & -1.0 & & & 1.0 & -1.0 & -1.2 & -1.3 & -1.5 & -1.6 & & -2.0 & -2.3 & \\
\hline Contribution from interest rate/growth differential & -1.3 & -0.9 & -0.2 & & & -0.9 & -1.2 & -1.5 & -1.6 & -1.7 & -1.8 & & -2.1 & -2.4 & \\
\hline of which: contribution from average real interest rate & -0.3 & -0.3 & 0.2 & & & 0.2 & 0.2 & 0.2 & 0.2 & 0.2 & 0.2 & & 0.1 & 0.1 & \\
\hline of which: contribution from real GDP growth & -1.0 & -0.6 & -0.4 & & & -1.1 & -1.4 & -1.7 & -1.8 & -1.9 & -2.0 & & -2.3 & -2.5 & \\
\hline Contribution from real exchange rate depreciation & -2.0 & 0.4 & -0.8 & & & 1.9 & 0.3 & 0.2 & 0.3 & 0.3 & 0.2 & & $\ldots$ & & \\
\hline Other identified debt-creating flows & -0.4 & -0.3 & -0.3 & & & -0.3 & -0.3 & -0.2 & -0.2 & -0.2 & -0.2 & & -0.1 & -0.1 & \\
\hline Privatization receipts (negative) & 0.0 & 0.0 & 0.0 & & & 0.0 & 0.0 & 0.0 & 0.0 & 0.0 & 0.0 & & 0.0 & 0.0 & \\
\hline Recognition of implicit or contingent liabilities & 0.0 & 0.0 & 0.0 & & & 0.0 & 0.0 & 0.0 & 0.0 & 0.0 & 0.0 & & 0.0 & 0.0 & \\
\hline Debt relief (HIPC and other) & -0.4 & -0.3 & -0.3 & & & -0.3 & -0.3 & -0.2 & -0.2 & -0.2 & -0.2 & & -0.1 & -0.1 & \\
\hline Other (specify, e.g. bank recapitalization) & 0.0 & 0.0 & 0.0 & & & 0.0 & 0.0 & 0.0 & 0.0 & 0.0 & 0.0 & & 0.0 & 0.0 & \\
\hline Residual, including as set changes & 1.9 & -2.7 & 3.9 & & & -0.7 & 0.3 & 0.1 & 0.1 & 0.0 & -0.7 & & 0.1 & 1.0 & \\
\hline \multicolumn{16}{|l|}{ Other $S$ ustainability Indicators } \\
\hline PV of public sector debt & 6.6 & 5.3 & 26.7 & & & 29.4 & 31.2 & 32.4 & 33.4 & 34.2 & 34.1 & & 36.2 & 35.4 & \\
\hline $\mathrm{o} / \mathrm{w}$ foreign-currency denominated & 0.0 & 0.0 & 18.7 & & & 20.8 & 21.1 & 21.3 & 21.5 & 20.9 & 21.0 & & 22.3 & 22.4 & \\
\hline $\mathrm{o} / \mathrm{w}$ external & $\ldots$ & $\ldots$ & 18.7 & & & 20.8 & 21.1 & 21.3 & 21.5 & 20.9 & 21.0 & & 22.3 & 22.4 & \\
\hline PV of contingent liabilities (not included in public sector debt) & $\ldots$ & & & & & $\ldots$ & $\ldots$ & $\ldots$ & & & & & $\ldots$ & & \\
\hline Gross financing need $2 /$ & 5.5 & 6.6 & 7.8 & & & 7.4 & 7.3 & 8.6 & 9.3 & 10.8 & 10.7 & & 10.2 & 7.5 & \\
\hline $\mathrm{PV}$ of public sector debt-to-revenue and grants ratio (in percent) & 27.8 & 24.3 & 123.0 & & & 135.1 & 143.1 & 146.4 & 149.5 & 151.4 & 149.5 & & 159.0 & 157.2 & \\
\hline PV of public sector debt-to-revenue ratio (in percent) & 31.7 & 27.6 & 145.5 & & & 154.1 & 161.4 & 164.8 & 167.9 & 169.7 & 167.3 & & 177.4 & 172.8 & \\
\hline o/w external 3/ & & & 101.6 & & & 109.1 & 108.8 & 108.3 & 108.2 & 103.7 & 103.4 & & 109.4 & 109.3 & \\
\hline Debt service-to-revenue and grants ratio (in percent) 4/ & 4.5 & 6.3 & 7.2 & & & 9.0 & 12.0 & 13.0 & 13.2 & 18.1 & 14.7 & & 14.3 & 17.2 & \\
\hline Debt service-to-revenue ratio (in percent) $4 /$ & 5.1 & 7.1 & 8.6 & & & 10.2 & 13.6 & 14.6 & 14.8 & 20.3 & 16.5 & & 15.9 & 18.9 & \\
\hline Primary deficit that stabilizes the debt-to-GDP ratio & 1.8 & 3.5 & -2.6 & & & 0.0 & 0.9 & 1.4 & 1.5 & 1.7 & 2.5 & & 2.1 & 1.4 & \\
\hline \multicolumn{16}{|l|}{ Key macroeconomic and fiscal assumptions } \\
\hline Real GDP growth (in percent) & 4.8 & 2.3 & 1.5 & 3.8 & 2.0 & 3.4 & 4.1 & 4.5 & 4.7 & 4.9 & 5.0 & 4.4 & 5.2 & 5.6 & 5.3 \\
\hline Average nominal interest rate on forex debt (in percent) & 1.4 & 1.6 & 1.7 & 1.4 & 0.3 & 2.3 & 2.0 & 1.8 & 1.7 & 1.6 & 1.3 & 1.8 & 1.2 & 1.2 & 1.2 \\
\hline Average real interest rate on domestic debt (in percent) & -1.4 & -2.9 & 6.2 & 0.6 & 3.5 & 2.7 & 3.8 & 3.8 & 3.8 & 3.8 & 3.8 & 3.6 & 3.8 & 3.7 & 3.8 \\
\hline Real exchange rate depreciation (in percent, + indicates depreciation) & -11.9 & 2.2 & -3.9 & -3.4 & 10.3 & 8.1 & $\ldots$ & & & & & & $\ldots$ & & $\ldots$ \\
\hline Inflation rate (GDP deflator, in percent) & 5.4 & 7.3 & -0.5 & 2.8 & 2.4 & 2.3 & 2.1 & 2.1 & 2.1 & 2.1 & 2.1 & 2.1 & 2.1 & 2.2 & 2.2 \\
\hline Growth of real primary spending (deflated by GDP deflator, in percent) & 0.1 & 0.0 & 0.0 & 0.1 & 0.1 & 0.0 & 0.0 & 0.0 & 0.1 & 0.1 & 0.1 & 0.0 & 0.0 & 0.0 & 0.0 \\
\hline Grant element of new external borrowing (in percent) & & $\ldots$ & $\ldots$ & $\ldots$ & $\ldots$ & 43.1 & 43.1 & 43.1 & 43.1 & 43.1 & 42.5 & 43.0 & 40.4 & 37.5 & $\ldots$ \\
\hline
\end{tabular}

Sources: Country authorities; and staff estimates and projections.

2/ Gross financing need is defined as the primary deficit plus debt service plus the stock of short-term debt at the end of the last period.

$3 /$ Revenues excluding grants.

4/ Debt service is defined as the sum of interest and amortization of medium and long-term debt.

$5 /$ Historical averages and standard deviations are generally derived over the past 10 years, subject to data availability. 
Table 4.Senegal: Sensitivity Analysis for Key Indicators of Public Debt 2010-2030

Projections

\begin{tabular}{llllllll}
2010 & 2011 & 2012 & 2013 & 2014 & 2015 & 2020 & 2030 \\
\hline
\end{tabular}

PV of Debt-to-GDP Ratio

Baseline

\section{A. Alternative scenarios}

A1. Real GDP growth and primary balance are at historical averages

A2. Primary balance is unchanged from 2010

A3. Permanently lower GDP growth 1/

B. Bound tests

B1. Real GDP growth is at historical average minus one standard deviations in 2011-2012

B2. Primary balance is at historical average minus one standard deviations in 2011-2012

B3. Combination of B1-B2 using one half standard deviation shocks

B4. One-time 30 percent real depreciation in 2011

B5. 10 percent of GDP increase in other debt-creating flows in 2011

PV of Debt-to-Revenue Ratio 2/

Baseline

$\begin{array}{llllllll}29 & 31 & 32 & 33 & 34 & 34 & 36 & 35\end{array}$

$\begin{array}{llllllll}29 & 30 & 31 & 32 & 32 & 32 & 33 & 38\end{array}$

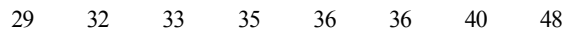

$\begin{array}{llllllll}29 & 31 & 33 & 34 & 35 & 36 & 41 & 50\end{array}$

\section{A. Alternative scenarios}

A1. Real GDP growth and primary balance are at historical averages

A2. Primary balance is unchanged from 2010

A3. Permanently lower GDP growth 1 /

\section{B. Bound tests}

B1. Real GDP growth is at historical average minus one standard deviations in 2011-2012

B2. Primary balance is at historical average minus one standard deviations in 2011-2012

B3. Combination of B1-B2 using one half standard deviation shocks

B4. One-time 30 percent real depreciation in 2011

B5. 10 percent of GDP increase in other debt-creating flows in 2011

Debt Service-to-Revenue Ratio 2

$\begin{array}{llllllll}29 & 32 & 35 & 37 & 39 & 39 & 44 & 47 \\ 29 & 32 & 34 & 35 & 36 & 36 & 38 & 36 \\ 29 & 32 & 33 & 35 & 36 & 37 & 41 & 42 \\ 29 & 40 & 40 & 40 & 40 & 39 & 39 & 35 \\ 29 & 39 & 40 & 41 & 41 & 40 & 41 & 38\end{array}$

$\begin{array}{llllllll}135 & 139 & 140 & 141 & 142 & 139 & 144 & 166 \\ 135 & 144 & 150 & 156 & 160 & 160 & 177 & 212\end{array}$

$\begin{array}{llllllll}135 & 144 & 148 & 153 & 157 & 157 & 180 & 218\end{array}$

$\begin{array}{llllllll}135 & 148 & 158 & 165 & 170 & 171 & 193 & 209 \\ 135 & 147 & 156 & 158 & 160 & 157 & 165 & 161 \\ 135 & 144 & 151 & 156 & 160 & 160 & 178 & 187 \\ 135 & 182 & 181 & 181 & 179 & 173 & 170 & 156 \\ 135 & 181 & 182 & 181 & 181 & 177 & 181 & 171\end{array}$

Baseline

\section{A. Alternative scenarios}

A1. Real GDP growth and primary balance are at historical averages A2. Primary balance is unchanged from 2010

A3. Permanently lower GDP growth 1/

\section{B. Bound tests}

B1. Real GDP growth is at historical average minus one standard deviations in 2011-2012

B2. Primary balance is at historical average minus one standard deviations in 2011-2012

B3. Combination of B1-B2 using one half standard deviation shocks

B4. One-time 30 percent real depreciation in 2011

B5. 10 percent of GDP increase in other debt-creating flows in 2011

Sources: Country authorities; and staff estimates and projections.

1/ Assumes that real GDP growth is at baseline minus one standard deviation divided by the square root of the length of the projection period.

2/ Revenues are defined inclusive of grants. 
Figure 3.Senegal: Indicators of Public Debt Under Alternative Scenarios, 2010-2030 1/
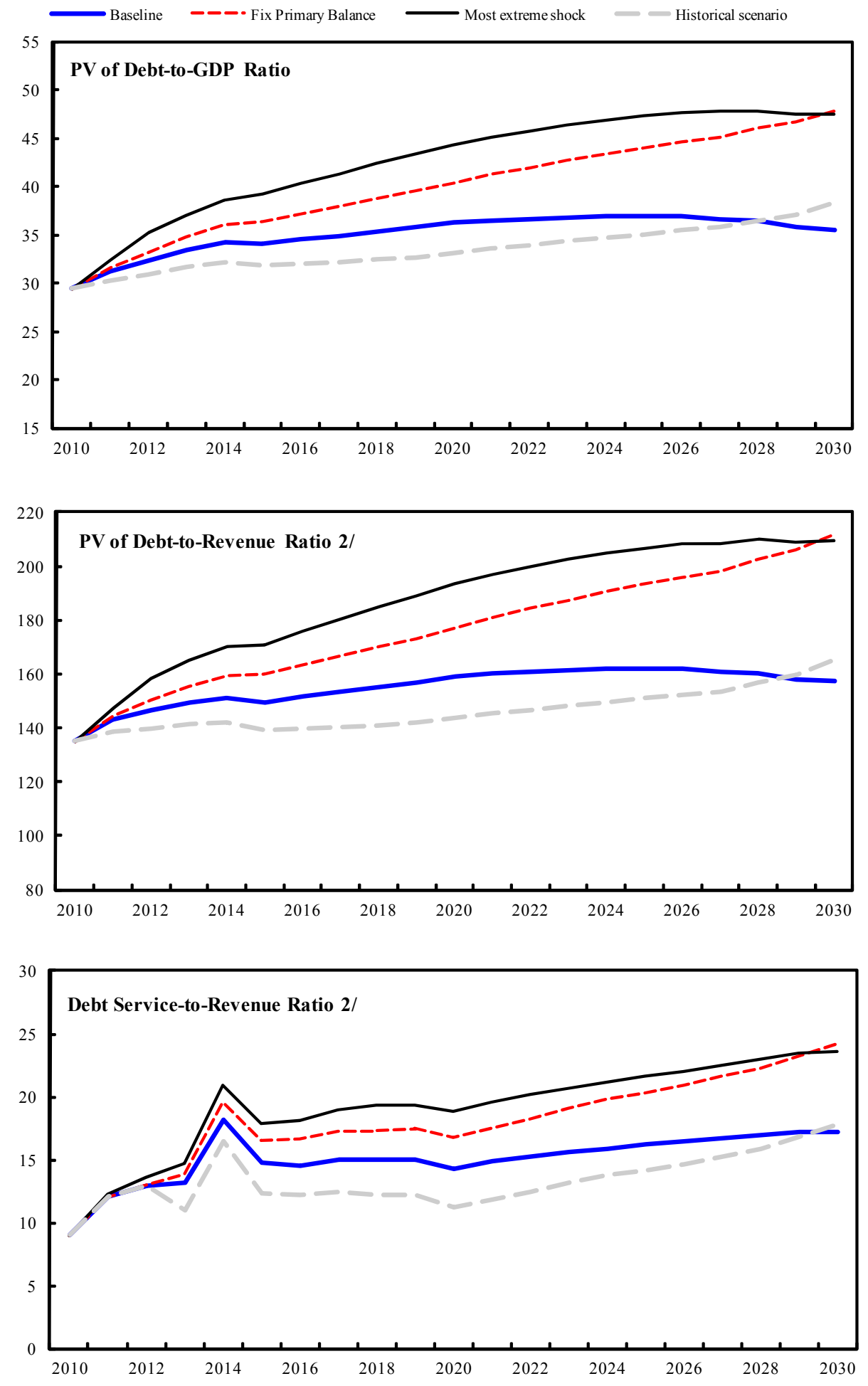

Sources: Country authorities; and staff estimates and projections.

1/ The most extreme stress test is the test that yields the highest ratio in 2020.

2/ Revenues are defined inclusive of grants. 


\section{SENEGAL}

Staff Report for the 2010 Article IV Consultation, Fifth Review Under the Policy Support Instrument, Third Review Under the Exogenous Shocks Facility, Request for Waiver of Nonobservance of Performance Criterion, and Modification of Assessment Criterion-Informational Annex

Prepared by the African Department

(In collaboration with other departments)

Approved by Roger Nord and Thomas Dorsey

May 7, 2010

- Relations with the Fund. Describes financial and technical assistance by the IMF and provides information on the safeguards assessment and exchange system. Outstanding purchases and loans amounted to SDR 106.32 million (65.71 percent of quota) at end-March 2010.

- JMAP Implementation. Describes Bank-Fund collaboration.

- $\quad$ Statistical Issues. Data provision has some shortcomings, but is broadly adequate for surveillance and program monitoring. There are weaknesses in data particularly on national accounts, production, and social indicators. 


\section{SENEGAL: RELATIONS WITH THE FUND \\ (As of March 31, 2010)}

I. Membership Status: Joined: August 31, 1962; Article VIII

II. General Resources Account:

Quota

Fund holdings of currency

Reserve Position

III. SDR Department:

Net cumulative allocation

Holdings

IV. Outstanding Purchases and Loans:

ESF Arrangements

PRGF Arrangements

SDR Million
161.80
160.08
1.74

SDR Million

154.80

130.37

SDR Million

88.99

17.33
$\%$ Quota

100.00

98.94

1.08

$\%$ Allocation

100.00

84.22

$\%$ Quota

55.00

10.71

\section{Latest Financial Arrangements:}

Type Date of Arrangement Expiration Date

$\begin{array}{lcccc}\text { Type } & \text { Date of Arrangement Expiration Date } & \begin{array}{c}\text { Amount Approved } \\ \text { (SDR Million) }\end{array} & \begin{array}{c}\text { Amount Drawn } \\ \text { (SDR Million) }\end{array} \\ \text { ESF } & \text { Dec 19, 2008 } & \text { Jun 18, 2010 } & \frac{121.35}{88.99} & 24.27 \\ \text { PRGF } & \text { Apr 28, 2003 } & \text { Apr 27, 2006 } & 24.27 & 96.47 \\ \text { PRGF } & \text { Apr 20, 1998 } & \text { Apr 19, 2002 } & 107.01 & \end{array}$

VI. Projected Payments to Fund

(SDR Million; based on existing use of resources and present holdings of SDRs):

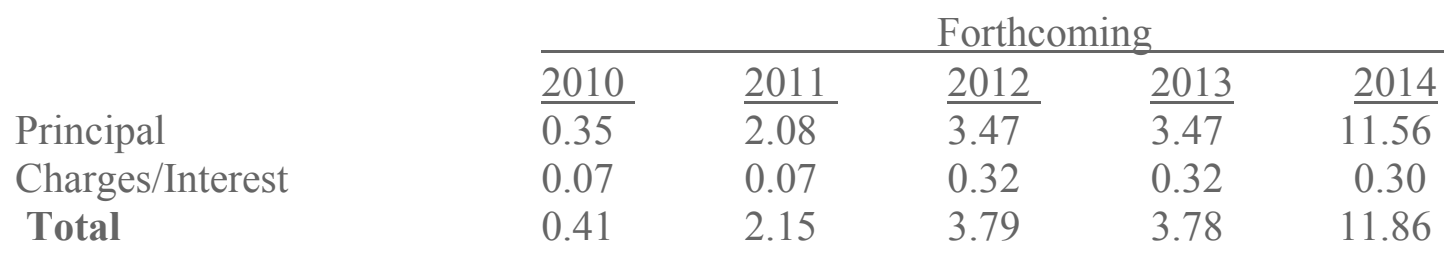

VII. Implementation of HIPC Initiative:

I. Commitment of HIPC assistance

Decision point date

Enhanced

Framework

June 2000 
Assistance committed by all creditors (US\$ Million) ${ }^{1}$

488.30

Of which: IMF assistance (US\$ million)

42.30

(SDR equivalent in millions)

33.80

Completion point date

April 2004

II. Disbursement of IMF assistance (SDR Million)

Assistance disbursed to the member

Interim assistance

14.31

Completion point balance

Additional disbursement of interest income ${ }^{2}$

Total disbursements

38.40

VIII. Implementation of Multilateral Debt Relief Initiative (MDRI):

I. $\quad$ MDRI-eligible debt (SDR Million) ${ }^{3}$

100.32

Financed by: MDRI Trust

94.76

Remaining HIPC resources

5.56

II. Debt Relief by Facility (SDR Million)

$\underline{\text { Delivery Date }}$

January 2006

GRA

N/A
EligibleDebt

PRGF

Total

100.32

100.32

\section{Safeguards Assessments:}

The Central Bank of West African States (BCEAO) is the common central bank of the countries of the West African Economic and Monetary Union, which includes Senegal. A safeguards assessment of the BCEAO was completed on November 4, 2005. The assessment indicated progress has been made in strengthening the bank's safeguards framework since the 2002 assessment and identified some areas where further steps would help solidify it.

\footnotetext{
${ }^{1}$ Assistance committed under the original framework is expressed in net present value (NPV) terms at the completion point, and assistance committed under the enhanced framework is expressed in NPV terms at the decision point. Hence the two amounts be added.

${ }^{2}$ Under the enhanced framework, an additional disbursement is made at the completion point corresponding to interest income earned on the amount committed at the decision point but not disbursed during the interim period.

${ }^{3}$ The MDRI provides 100 percent debt relief to eligible member countries that qualified for the assistance. Grant assistance from the MDRI Trust and HIPC resources provide debt relief to cover the full stock of debt owed to the Fund as of end-2004 that remains outstanding at the time the member qualifies for such debt relief.
} 
The BCEAO now publishes a full set of audited financial statements and improvements have been made to move financial reporting closer to International Financial Reporting Standards (IFRS). Furthermore, an internal audit charter has been put in place, mechanisms for improving risk management have been established, and follow-up on internal and external audit recommendations has been strengthened.

The most recent safeguards assessment of the BCEAO was completed on March 1, 2010. The 2010 update assessment found that the BCEAO continues to have controls in place at the operational level. The overall governance framework should nonetheless be strengthened by the addition of an audit committee to ensure that the Board of Directors exercises appropriate oversight over the control structure, including the audit mechanisms and financial statements. The upcoming implementation (2010) of the Institutional Reform of the WAMU and the BCEAO should help correct that situation. Efforts to implement fully the International Financial Reporting Standards reporting framework should also be pursued.

\section{Exchange System:}

Senegal is a member of the West African Economic and Monetary Union (WAEMU). The exchange system, common to all members of the union, is free of restrictions on the making of payments and transfers for current international transactions. The union's common currency, the CFA franc, had been pegged to the French franc at the rate of CFAF 1 = F 0.02. Effective January 12, 1994, the CFA franc was devalued and the new parity set at CFAF $1=$ F 0.01. Effective December 31, 1998, the parity was switched to the euro at a rate of CFAF $655.96=€ 1$.

The authorities confirmed that Senegal had not imposed measures that could give rise to exchange restrictions subject to Fund jurisdiction. They will inform the Fund if any such measure is introduced.

Aspects of the exchange system are also discussed in the February 2010 report on economic developments and regional policy issues of the WAEMU.

\section{Article IV Consultations:}

The previous 2008 Article IV consultation was completed by the Executive Board on June 18, 2008 (Country Report No.08/209). In concluding the 2008 Article IV consultation, Executive Directors encouraged the authorities to maintain prudent macroeconomic policies and persevere in implementing their structural reforms to encourage private-sector led growth. They considered that Senegal's sluggish export performance over the last decade was largely related to structural impediments in the economy, and encouraged the authorities to improve the business environment to make it more conducive to private-sector led growth, raise external competitiveness, and strengthen and diversify exports. They underlined that it was critical to contain the fiscal deficit to preserve debt sustainability, respect the limited financing capacity of the regional financial market, and help contribute to domestic stability in the WAEMU. Directors urged the careful review and expeditious settlement of payment delays, with a view to rigorously applying the existing budget framework. They welcomed the authorities' commitment to continue public financial management reform. 


\section{Financial Sector Assessment Program (FSAP) and Report on the Observance of Standards and Codes (ROSC) Participation:}

A joint team of the World Bank and the International Monetary Fund conducted a mission under the FSAP program in November 2000 and January 2001. The Financial System Stability Assessment (FSSA) was issued in August 2001 (IMF Country Report No. 01/189). An FSAP update was undertaken in June 2004, focusing on development issues (in particular nationwide supply of basic financial services and access of SMEs to credit), in line with the priorities defined in the PRSP (IMF Country Report No. 05/126). A regional FSAP for the WAEMU was undertaken in the second half of 2007.

A ROSC on the data module, based on a September 2001 mission, was published on December 2, 2002. An FAD mission conducted a ROSC on the fiscal transparency module in January 2005.

\section{Technical Assistance:}

\section{A. AFRITAC West}

\begin{tabular}{|c|c|c|}
\hline Year & Area & Focus \\
\hline 2003 & $\begin{array}{l}\text { Debt management and financial } \\
\text { markets } \\
\text { Microfinance }\end{array}$ & $\begin{array}{l}\text { Upgrading of information systems; techniques of external } \\
\text { debt management } \\
\text { Initiate work with BCEAO and donors }\end{array}$ \\
\hline 2004 & $\begin{array}{l}\text { Public expenditure management } \\
\text { Debt management and financial } \\
\text { markets } \\
\text { Public expenditure management } \\
\text { Debt management and financial } \\
\text { markets }\end{array}$ & $\begin{array}{l}\text { Workshop } \\
\text { Evaluation of software for improving debt management; } \\
\text { workshop on AFL/CFT } \\
\text { Decentralization; evaluation of TA needs } \\
\text { Assessing need for capacity improvement }\end{array}$ \\
\hline 2005 & $\begin{array}{l}\text { Macroeconomic statistics } \\
\text { Microfinance }\end{array}$ & $\begin{array}{l}\text { Making fiscal data conform to WAEMU and other } \\
\text { international norms } \\
\text { Inspection and control; workshop on good governance; } \\
\text { training of government supervisory personnel }\end{array}$ \\
\hline 2006 & $\begin{array}{l}\text { Customs administration } \\
\text { Tax administration } \\
\text { Macroeconomic statistics } \\
\text { National accounts } \\
\text { Microfinance }\end{array}$ & $\begin{array}{l}\text { Software risks } \\
\text { Reforms and TA needs } \\
\text { Evaluating implementation of prior TA and future needs } \\
\text { Work program for improvement and statistical action plan } \\
\text { Supervision }\end{array}$ \\
\hline 2007 & $\begin{array}{l}\text { Customs administration } \\
\text { Tax administration } \\
\text { Debt management and financial } \\
\text { markets }\end{array}$ & $\begin{array}{l}\text { Risk analysis and control } \\
\text { Modernization } \\
\text { Assessing TA needs; regional workshop on external debt } \\
\text { statistics }\end{array}$ \\
\hline
\end{tabular}




\begin{tabular}{|c|c|c|}
\hline Year & Area & Focus \\
\hline & Macroeconomic statistics & Public finance statistics \\
\hline & National accounts & $\begin{array}{l}\text { Institutional sectors and quarterly national accounts; } \\
\text { regional workshop on government accounts }\end{array}$ \\
\hline & Microfinance & Supervision \\
\hline \multirow[t]{3}{*}{2008} & $\begin{array}{l}\text { Debt management and financial } \\
\text { markets }\end{array}$ & DSA workshop \\
\hline & National accounts & Institutional sector and quarterly national accounts \\
\hline & Microfinance & Supervision and organization \\
\hline \multirow[t]{5}{*}{2009} & National accounts & Quarterly national accounts (QNA) \\
\hline & Tax administration & Status of the reform and scope for further TA \\
\hline & Debt management & Strengthening public debt management \\
\hline & Microfinance & Strengthening microfinance supervision \\
\hline & $\begin{array}{l}\text { Macroeconomic and financial } \\
\text { statistics }\end{array}$ & $\begin{array}{l}\text { Enhancing production and dissemination of public finances } \\
\text { statistics }\end{array}$ \\
\hline \multirow[t]{3}{*}{2010} & Debt management & Strengthening public debt management \\
\hline & National accounts & Quarterly national accounts (QNA) \\
\hline & Customs administration & Follow-up mission \\
\hline
\end{tabular}

\section{B. Headquarters}

\begin{tabular}{llll}
\hline Department & Date & Form & Purpose \\
\hline
\end{tabular}

Fiscal Affairs September 2001 Staff/consultant

$\begin{array}{lll}\text { February 2004 } & \text { Staff } & \text { reducing expenditures } \\ \text { November 2004 } & \text { Staff } & \text { PSIA-Poverty and social impact analysis } \\ \text { January 2005 } & \text { Staff } & \text { ROSC } \\ & & \\ \text { January 2008 } & \text { Staff } & \text { Public-Private Partnerships } \\ \text { February 2008 } & \text { Staff } & \text { PSIA-Poverty and Social Impact Analysis } \\ \text { October 2008 } & \text { Staff/AFRITAC } & \text { Public financial management }\end{array}$




\begin{tabular}{|c|c|c|c|}
\hline Department & Date & Form & Purpose \\
\hline & $\begin{array}{l}\text { April } 2009 \\
\text { November } 2009 \\
\text { January } 2010 \\
\text { February } 2010\end{array}$ & $\begin{array}{l}\text { FAD Expert } \\
\text { Staff/AFRITAC } \\
\text { FAD Expert } \\
\text { Staff/AFRITAC }\end{array}$ & $\begin{array}{l}\text { Public financial management } \\
\text { Revenue administration } \\
\text { Review of the expenditure chain } \\
\text { Public financial management }\end{array}$ \\
\hline $\begin{array}{l}\text { Monetary and } \\
\text { Capital } \\
\text { Markets }\end{array}$ & September 2006 & Staff & Bank supervision and regulation \\
\hline \multirow[t]{10}{*}{ Statistics } & September 2001 & Staff & ROSC assessment of data \\
\hline & July 2002 & AFRISTAT & $\begin{array}{l}\text { Real sector statistics assessment under } \\
\text { GDDS West Africa project }\end{array}$ \\
\hline & August 2002 & AFRISTAT & $\begin{array}{l}\text { National accounts assistance under GDDS } \\
\text { West Africa project. }\end{array}$ \\
\hline & August 2002 & $\begin{array}{l}\text { Regional } \\
\text { advisor }\end{array}$ & $\begin{array}{l}\text { Continued assistance with fiscal sector data } \\
\text { under GDDS West Africa project. }\end{array}$ \\
\hline & December 2002 & AFRISTAT & $\begin{array}{l}\text { Continued assistance with national } \\
\text { accounts and prices statistics under GDDS } \\
\text { West Africa project }\end{array}$ \\
\hline & February 2003 & $\begin{array}{l}\text { Regional } \\
\text { advisor }\end{array}$ & $\begin{array}{l}\text { Continued assistance with fiscal sector data } \\
\text { under GDDS West Africa project. }\end{array}$ \\
\hline & March 2006 & Staff & Real sector statistics \\
\hline & March 2006 & Staff & Government finance statistics \\
\hline & November 2008 & Staff & SDDS assessment \\
\hline & April 2009 & Staff & Government finance statistics \\
\hline
\end{tabular}

\section{Resident Representative}

Stationed in Dakar since July 24, 1984. The position has been held by Ms. Valeria Fichera since September 2009.

\section{Anti Money Laundering / Combating the Financing of Terrorism}

The onsite visit for Senegal's AML/CFT evaluation took place in July/August 2007 in the context of ECOWAS Inter-Governmental Action Group Against Money Laundering (GIABA). The report was adopted in early May 2008 by the GIABA Plenary held in Accra, Ghana. The report highlighted several areas of weaknesses in the AML/CFT system, confirmed by the score of 12 Non-Compliant and 16 Partially Compliant ratings out of the $40+9$ FAF AML/CFT recommendations. GIABA's first follow up report on the implementation of the recommendations contained in the Mutual Evaluation (2009) mentions that Senegal's adoption of Uniform Law No. 2009-16 of 02 March 2009 against terrorist 
financing enables the country to broadly comply with all the Recommendations and special recommendations concerning the issue, including customer Due Diligence (especially as regards politically exposed persons (PEP). It also notes that legal provisions made by Senegal in order to prevent the abuse of new technologies, namely the adoption of Law No. 2008-11 of 25 January 2008 on cyber criminality, enable the country to adapt its criminal system and subsequent procedures to crimes related to new information and communication technologies. GIABA Secretariat concludes that Senegal deserves encouragement for its endeavour to reinforce its AML/CFT scheme and recommends, at this juncture, the maintenance of Senegal within a regular follow-up process, pending the results of measures taken and the adoption of new measures aimed at amending the above-mentioned scheme. 


\section{Joint Management ACtion Plan IMPlementation}

World Bank and International Monetary Fund Collaboration

\begin{tabular}{|c|c|c|c|}
\hline Title & Products & $\begin{array}{c}\text { Provisional timing of } \\
\text { missions }\end{array}$ & Expected delivery date \\
\hline \multicolumn{4}{|c|}{ A. Mutual information on relevant work programs } \\
\hline World Bank & $\begin{array}{l}\text { Public Expenditure Review } \\
\text { Poverty Reduction Support } \\
\text { Credit IV }\end{array}$ & $\begin{array}{l}\text { Continuous } \\
\text { July 2009-March } 2010\end{array}$ & May 2010 (Board) \\
\hline \multirow{4}{*}{$\begin{array}{l}\text { International } \\
\text { Monetary Fund }\end{array}$} & $\begin{array}{l}\text { IMF-supported program } \\
\text { Sixth Review of PSI }\end{array}$ & September 2010 & October 2010 (Board) \\
\hline & $\begin{array}{l}\text { Technical Assistance } \\
\text { Public financial } \\
\text { management }\end{array}$ & February 2010 & May 2010 \\
\hline & $\begin{array}{l}\text { Tax administration follow- } \\
\text { up mission }\end{array}$ & November 2010 & \\
\hline & $\begin{array}{l}\text { Two short-term expert } \\
\text { visits on accounting and } \\
\text { cash-management }\end{array}$ & First half of FY 2011 & \\
\hline \multicolumn{4}{|c|}{ B. Requests for work program inputs } \\
\hline $\begin{array}{l}\text { Fund request to } \\
\text { Bank (with } \\
\text { summary } \\
\text { justification) }\end{array}$ & $\begin{array}{l}\text { Updates on progress with } \\
\text { PRSC (if implications for the } \\
\text { IMF-supported program) } \\
\text {.Energy sector reform }\end{array}$ & $\ldots$ & $\ldots$ \\
\hline $\begin{array}{l}\text { Bank request to } \\
\text { Fund (with } \\
\text { summary } \\
\text { justification) }\end{array}$ & $\ldots$ & $\ldots$ & $\ldots$ \\
\hline \multicolumn{4}{|c|}{ C. Agreement on joint products and missions } \\
\hline Joint products & DSA & March-April 2011 & May 2011 \\
\hline
\end{tabular}




\section{STATISTICAL ISSUES}

\section{Senegal - Statistical Issues Appendix}

As of April 13, 2010

\section{Assessment of Data Adequacy for Surveillance}

General: Data provision has some shortcomings, but is broadly adequate for surveillance and program monitoring. There are weaknesses in data on national accounts, production, and social indicators. The authorities are committed to improving the quality and availability of economic, financial and social indicators, partially relying on technical assistance from the Fund and other international organizations and donors.

National accounts: The compilation of the national accounts generally follows the System of National Accounts, 1993. Despite staff's professionalism, the lack of adequate financial resources has constrained efforts to collect and process data. Data sources are deficient in some areas, particularly the informal sector. Owing to financial constraints, surveys of business and households are not conducted regularly. However, efforts are being made to improve data collection procedures, strengthen the coordination among statistical agencies, and reduce delays in data dissemination. The Regional Technical Assistance Center for West Africa (West AFRITAC) has been assisting member countries, including Senegal, with the improvement of their real sector statistics, in particular annual and quarterly national accounts (QNA). Progress reported by the advisor includes: i) completion of national accounts for 1980-2004 with 1999 as the base year; ii) dissemination of the 1980-2003 series in hard copy and on the internet; iii) production of accounts by institutional sector (first series covers 2004 institutional sector accounts); and iv) production of national accounts in accordance with the dissemination schedule. The authorities plan to start production of quarterly national accounts in view of the country's intention to subscribe to the SDDS. The recent West AFRITAC missions have assisted with training to support compilation of the QNA and initiating their compilation for the period 1990-2007. The West AFRITAC and the authorities agreed on a detailed work program initially aimed at starting regular dissemination of the QNA in March 2010. A stocktaking mission is scheduled in April 2010.

Government finance statistics (GFS): GFS are compiled by the Ministry of Economy and Finance from customs, tax, and treasury directorate sources. Data last reported to STA for electronic redissemination and publication in the 2007 Government Finance Statistics Yearbook were for fiscal year 2001. Higher frequency data are not provided for redissemination in $I F S$, but the ministry compiles and disseminates quarterly government financial operations tables (TOFE) in their own publications. An AFR team worked with the authorities in February 2004 to improve fiscal reporting in the context of the last PRGF-supported program. The team focused on (i) public accounts that are outside of the direct purview of the treasury; (ii) the treatment of correspondents' accounts in the TOFE; and (iii) ensuring consistency between treasury and banking system information concerning government transactions. The proposed changes are now being implemented. They have improved the presentation of government financial operations and are the first step toward bringing the TOFE more in line with the extended WAEMU TOFE. Other steps will include implementing the WAEMU fiscal directives that are being revised. A regional advisor in GFS has been conducting technical assistance missions aimed at improving the consistency of fiscal reporting and migrating to the methodologies of the Government Finance Statistics Manual 2001. The regional advisor also supported efforts to resume reporting of annual and higher frequency data for publication in International Financial Statistics (IFS) and electronic dissemination of the GFS Yearbook. 
Monetary and financial statistics: Preliminary monetary data are compiled by the national agency of the Central Bank of West African States (BCEAO) and officially released (including to the IMF) by BCEAO headquarters. The authorities are now reporting monetary data to STA on a regular basis, with a reduction in the lag from about six months to about three to four months. There has also been an improvement in the timeliness of reporting interest rate and main depository corporation data (central bank, commercial banks and postal checks center). An area-wide page for the WAEMU zone was introduced in the January 2003 issue of IFS. In 2005, the BCEAO made substantial revisions to the estimates of banknotes in circulation in member states resulting from cross-border banknote movement. These revisions were due to changes in the method to estimate currency in circulation in the WAEMU countries. The revised method, based on updated sorting coefficients ("coefficients de tri"), has been applied retroactively from December 2003. In August 2006, as part of the authorities' continuing efforts to implement the statistical methodology recommended in the Monetary and Financial Statistics Manual, the BCEAO reported to STA test monetary data for June 2006 for all member countries using the Standardized Report Forms (SRF). In response to STA's comments, the BCEAO has provided a revised central bank report form (1SR) as well as test data on other depository corporations (2SR) for review by STA.

External sector statistics: Balance of payments statistics are compiled by the Senegalese national agency of the BCEAO. With STA support over the past few years, several steps have been taken to address certain shortcomings, including: (i) implementation of the Balance of Payments Manual, fifth edition; (ii) modification and simplification of related surveys for companies and banks; (iii) improvement in the computerization of procedures; and (iv) significant strengthening of staff training. Nevertheless, further steps could be taken to enhance the quality and coverage of the balance of payments statistics. Although definitive balance of payments statistics can now be provided with a delay of less than one year, there are significant delays in reporting the data to STA.

\section{Data Standards and Quality}

The country has begun the process of regional harmonization of statistical methodologies within the framework of the WAEMU. It participates in the General Data Dissemination System A Data ROSC was published on the IMF website on (GDDS), and its metadata were posted on the Fund's Dissemination Standards Bulletin Board on September 10, 2001. In September 2006, the authorities expressed their commitment to work toward subscription to the Special Data Dissemination Standard (SDDS) and have appointed a national SDDS coordinator. The November 2008 SDDS assessment mission evaluated dissemination practices against SDDS requirements for coverage, periodicity and timeliness and, in cooperation with the authorities, developed an action plan to address identified gaps. 
Senegal: Table of Common Indicators Required for Surveillance

(As of April 13, 2010)

\begin{tabular}{|c|c|c|c|c|c|c|c|}
\hline & \multirow[b]{2}{*}{$\begin{array}{c}\text { Latest } \\
\text { observation }\end{array}$} & \multirow[b]{2}{*}{$\begin{array}{l}\text { Date } \\
\text { received }\end{array}$} & \multirow[b]{2}{*}{$\begin{array}{l}\text { Frequency } \\
\text { of data }^{7}\end{array}$} & \multirow[b]{2}{*}{$\begin{array}{l}\text { Frequency of } \\
\text { reporting }\end{array}$} & \multirow[b]{2}{*}{$\begin{array}{l}\text { Frequency of } \\
\text { publication }^{7}\end{array}$} & \multicolumn{2}{|c|}{ Memo Items: } \\
\hline & & & & & & $\begin{array}{c}\text { Data Quality - } \\
\text { Methodological } \\
\text { soundness }\end{array}$ & $\begin{array}{c}\text { Data Quality Accuracy } \\
\text { and reliability }\end{array}$ \\
\hline Exchange Rates & Current & Current & $\mathrm{D}$ & $\mathrm{D}$ & $\mathrm{D}$ & & \\
\hline $\begin{array}{l}\text { International Reserve Assets and Reserve Liabilities of the Monetary } \\
\text { Authorities }{ }^{1}\end{array}$ & $2 / 2010$ & $3 / 2010$ & M & M & M & & \\
\hline Reserve/Base Money & $1 / 2010$ & $3 / 2010$ & M & M & M & \multirow{4}{*}{$\mathrm{LO}, \mathrm{LO}, \mathrm{O}, \mathrm{O}$} & \multirow{4}{*}{$\mathrm{LO}, \mathrm{O}, \mathrm{O}, \mathrm{LO}$} \\
\hline Broad Money & $1 / 2010$ & $3 / 2010$ & M & M & M & & \\
\hline Central Bank Balance Sheet & $2 / 2010$ & $3 / 2010$ & M & M & M & & \\
\hline Consolidated Balance Sheet of the Banking System & $12 / 2009$ & $3 / 2010$ & M & M & M & & \\
\hline Interest Rates ${ }^{2}$ & $2 / 2010$ & $3 / 2010$ & M & M & M & & \\
\hline Consumer Price Index & $2 / 2010$ & $3 / 2010$ & M & M & M & $\mathrm{O}, \mathrm{LO}, \mathrm{O}, \mathrm{O}$ & LO, O, O, NA \\
\hline $\begin{array}{l}\text { Revenue, Expenditure, Balance and Composition of Financing }{ }^{3}-\text { General } \\
\text { Government }{ }^{4}\end{array}$ & NA & NA & & & & \multirow[b]{2}{*}{$\mathrm{O}, \mathrm{LNO}, \mathrm{LO}, \mathrm{O}$} & \multirow[b]{2}{*}{$\mathrm{LO}, \mathrm{LO}, \mathrm{O}, \mathrm{LO}$} \\
\hline $\begin{array}{l}\text { Revenue, Expenditure, Balance and Composition of Financing }{ }^{3}-\text { Central } \\
\text { Government }\end{array}$ & $2 / 2010$ & $3 / 2010$ & M & M & M & & \\
\hline Stocks of Central Government and Central Government-Guaranteed Debt $t^{5 / 11}$ & 2009 & $3 / 2010$ & & & & & \\
\hline External Current Account Balance ${ }^{10 / 11}$ & 2009 & $3 / 2010$ & A & A & A & \multirow[b]{2}{*}{$\mathrm{O}, \mathrm{O}, \mathrm{O}, \mathrm{O}$} & \multirow[b]{2}{*}{$\mathrm{O}, \mathrm{O}, \mathrm{O}, \mathrm{O}$} \\
\hline Exports and Imports of Goods and Services ${ }^{10 / 11}$ & 2009 & $3 / 2010$ & A & A & A & & \\
\hline GDP/GNP ${ }^{10 / 11}$ & 2009 & $3 / 2010$ & A & I & A & LO, LO, LO, LNO & LNO, LNO, LNO, LNO \\
\hline Gross External Debt ${ }^{11}$ & 2009 & $3 / 2010$ & $\mathrm{~A}$ & I & A & & \\
\hline International Investment Position ${ }^{6 /}$ & 2007 & $4 / 2009$ & A & A & A & & \\
\hline
\end{tabular}

${ }^{1}$ Includes reserve assets pledged or otherwise encumbered as well as net derivative positions.

${ }^{2}$ Both market-based and officially-determined, including discount rates, money market rates, rates on treasury bills, notes and bonds.

${ }^{3}$ Foreign, domestic bank, and domestic nonbank financing.

${ }^{4}$ The general government consists of the central government (budgetary funds, extra budgetary funds, and social security funds) and state and local governments.

${ }^{5}$ Including currency and maturity composition.

${ }^{6}$ Includes external gross financial asset and liability positions vis-à-vis nonresidents.

${ }^{7}$ Daily (D); Weekly (W); Monthly (M); Quarterly (Q); Annually (A); Irregular (I); Not Available (NA).

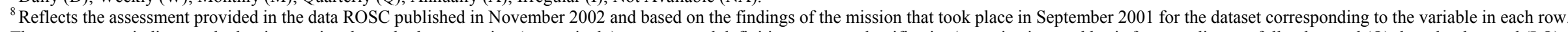

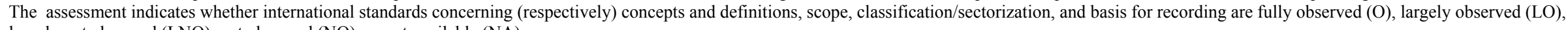
largely not observed (LNO), not observed (NO), or not available (NA).

${ }^{9}$ Same as footnote 8, except referring to international standards concerning (respectively) source data, statistical techniques, assessment and validation of source data, and revision studies.

${ }^{10}$ Estimate.

${ }^{11}$ Reported to staff during mission. 
Press Release No. $10 / 212$

FOR IMMEDIATE RELEASE

May 24, 2010
International Monetary Fund

Washington, D.C. 20431 USA

\section{IMF Completes Third and Final Review Under Senegal's ESF Arrangement, Approves US\$47.7 Million Disbursement and Completes Fifth Review Under PSI}

The Executive Board of the International Monetary Fund (IMF) completed the third and final review of Senegal's performance under an economic program supported by an Exogenous Shock Facility (ESF) arrangement. The approval enables Senegal to draw the remaining amount equivalent to SDR 32.36 million (about US $\$ 47.7$ million), bringing total disbursements under the ESF arrangement to SDR 121.35 million (about US\$178.8 million). The Executive Board also approved waivers for the nonobservance of the performance criteria on the basic fiscal balance and nonconcessional external debt.

The ESF arrangement for Senegal was approved on December 19, 2008 (see Press Release No. 08/334) to help the country meet a larger balance of payments need brought about by higher food and energy prices. On June 19, 2009, the Executive Board approved a financial increase under the ESF arrangement by SDR 72.81 million (about US\$107.3 million) to SDR 121.35 million (about US\$178.8 million) and an extension of the arrangement from 12 to 18 months to help finance the balance-of-payments impact of the global economic crisis (See Press Release No. 09/223).

The Executive Board has also completed the fifth review under the Policy Support Instrument (PSI) for Senegal. The three-year PSI for Senegal was approved on November 2, 2007 (see Press Release No. 07/246) to support the country's economic reform efforts. It is aimed at consolidating macroeconomic stability, increasing the country's growth potential, and reducing poverty. The Executive Board also approved a waiver for the nonobservance of the assessment criterion on the basic fiscal balance.

Following the Executive Board discussion on Senegal, Mr. Murilo Portugal, Deputy Managing Director and Acting Chair, stated:

"Following food and fuel price shocks in 2008, economic activity slowed further in 2009 because of the global economic downturn and domestic shocks, including temporary electricity shortages. While some uncertainties about the economic outlook persist, recent 
indicators suggest that economic growth may have bottomed out. Growth is projected to gradually recover. Consumer price inflation, which was negative in the second half of 2009 due to international price developments and the absence of domestic demand pressures, is expected to return to its long-run trend.

"The government is envisaging medium-term fiscal consolidation and continuing to fully normalize relations with the private sector. While the risks of debt distress are low, the temporary fiscal stimulus will need to be gradually reduced to reach the deficit target consistent with debt sustainability. The government is committed to a swift and transparent settlement of remaining extrabudgetary spending and agency debt. This should support the economic recovery and allow more space for the government to pursue its objectives of raising growth-enhancing public investments and sheltering priority spending.

"It will be important to continue with structural reforms to help achieve the objective of raising growth. Further public financial management reforms are needed to make expenditure planning and control more effective. Investment projects should be selected and prioritized based on rigorous economic cost-benefit analysis to raise the productivity of government spending. The energy sector reform plan needs to be fully and promptly implemented to limit fiscal and economic risks. Sustained efforts are also required to enhance the financial sector's contribution to the economy." 


\title{
Statement by Laurean Rutayisire, Executive Director and Kossi Assimaidou, Alternate Executive Director for Senegal
}

\author{
May 19, 2010
}

1. We thank the staff for a balanced report. Since the last program review, the authorities have continued to forcefully implement their economic program supported by the Policy Support Instrument (PSI) and the Exogenous Shocks Facility (ESF) with a view to making further inroads into poverty reduction through strong growth and good economic governance. After growth performance weakened last year largely on account of a slowdown in FDI inflows and reduced external demand, there is now scope for cautious optimism about growth prospects in view of latest economic developments. Indeed, clear signs of economic recovery are emerging, underpinning growth projections for 2010 which more than double last year's estimates.

\section{Program Performance}

2. Since the last PSI and ESF reviews, program developments have been broadly positive. Program implementation has been characterized on the quantitative front by the observance of four out of five quantitative criteria set forth by the program at end-December 2009. Notably the authorities have fully discontinued the use of Treasury cash advances, contained the budgetary float, maintained prudent management of external debt, and have exercised limit on recourse to single-tender contracts. However, the criterion on the basic fiscal balance was missed following a combination of lower-than-projected revenues and higher-than-expected current expenditures which were notably aimed at addressing urgent needs, particularly in the education sector, and entailed the use of legally permissible supplemental appropriation decrees. Going forward, the authorities are determined to limit recourse to such decrees and to accommodate any resulting spending increase by reducing nonpriority expenditures. In this light, we would welcome Directors' support of the authorities' request for a waiver of nonobservance of this criterion.

3. In line with commitments made on the structural front, the authorities took steps to comply with existing budgetary procedures, essentially ensuring timely execution and accounting closure of the 2009 budget and systematically publishing budgetary and fiscal tables. They also made continuous efforts to clear the remaining extrabudgetary commitments and debt contracted by public institutions and agencies. In this connection, settlement of part of these liabilities was already programmed in the 2009 and 2010 budgets. Moreover, these liabilities were audited last year and a summary of the results made public. The authorities have elaborated and submitted to the parliament a supplementary budget for 2010 that accounts for the settlement of extrabudgetary commitments as well as part of remaining debt of public institutions and agencies.

4. After completing a study according to which estimated tax expenditures and other tax exemptions could amount to over 3 percent of GDP, the authorities worked forcefully on 
developing an action plan aimed at reducing them. This plan was finalized according to schedule and some of the proposed changes are expected to be reflected in the next budget. Going forward, the authorities have stated their intention to ensure that any new tax expenditures will have to go through successfully an impact assessment prior to its approval.

5. In the financial sector, the authorities made progress in following up with FSAP recommendations. In this regard, consolidated action plan was finalized to implement these recommendations notably with the aim at promoting financial sector development and intermediation, strengthening the legal and institutional framework of the sector, and safeguarding financial stability.

\section{Policy and Reform Agenda for 2010 and 2011}

6. Macroeconomic policy will continue to aim at laying down the basis for continued private sector development and progress toward poverty reduction while taking care to safeguard fiscal and debt sustainability and financial stability. In particular, this policy orientation is mirrored on the fiscal front by the budgeted increase in social expenditure from 33 percent of total expenditures in 2008 to 35 percent in 2010. At the same time, efforts to close the infrastructure gap will be pursued and to this end additional fiscal space is expected to be freed, among others, by the projected decline in current expenditures by about 0.8 percent of GDP between 2009 and 2010. Overall, fiscal policy will be conducted with prudence, taking into account potential adverse implications of domestic and external developments for revenue performance and financing prospects. Reflecting the authorities' attachment to fiscal prudence, program fiscal targets have been revised on the basis of more conservative assumptions about revenue and financing. Over the medium term, the authorities remain firmly committed to constraining the overall fiscal deficit to 4 percent of GDP over the medium term.

7. The authorities attach a high premium to continued improvements in public financial management (PFM) and high value to Fund technical assistance in this area. As rightly underscored in the staff report, the authorities' positive reaction to Fund advice materialized inter alia into the launch of far-reaching PFM reforms building notably on the recommendations formulated by Fund technical assistance missions. The authorities' PFM reform agenda also sets forth further improvements in the budget monitoring system (SIGFIP) notably through the inclusion of payroll data and the implementation of an interface between SIGFIP and the Treasury accounting system (ASTER).

8. Relations with the private sector will be further normalized, notably with the planned clearance of at least 50 percent of the estimated remaining unsettled extrabudgetary spending and public institutions and agencies' debt. In parallel, an audit will be carried out to ascertain if new extrabudgetary commitments were made or additional debt accumulated by public institutions and agencies since end-2008. The results of this audit will be made public along with the status of the clearance process and the prospective steps to be taken to make these extrabudgetary expenditures and agency debt history. It is expected that the new reporting mechanisms which are now in place in public agencies and institutions, coupled with improved management of their cash flow transactions at the Treasury, will facilitate 
monitoring and strengthening their liquidity situation, thereby helping them avoid accumulation of new debt.

9. More generally, the authorities are taking steps to improve liquidity management by finalizing a strategy for establishing a Treasury single account. In this respect, an exhaustive census of government accounts is being updated and the Treasury's liquidity forecasting capacities are intended to be upgraded.

10. Among other main areas covered by the authorities' ambitious reform agenda are the reforms of the energy and financial sectors. In the energy sector, efforts that are underway are geared towards strengthening performance and the financial and debt situation of the electricity company, SENELEC. To this end, the authorities are working in close collaboration with key country's partners, including the World Bank, with a view to disaggregating SENELEC's activities into production, transport, and distribution of electricity. To ensure improved tax compliance of the company, the envisaged implementation of legal and regulatory measures will be helpful.

11. With regard to the financial sector, the encompassing reform agenda set forth in the government action plan will be pursued. In particular, it will entail continued, close monitoring of banks' soundness indicators and introduction of new minimum capital rules, conduct of regular stress tests, and capacity building at the finance ministry's unit in charge of regulating and supervising microfinance institutions.

12. The authorities' attention was recently drawn on the fact that the grant element of a loan equivalent to less than 0.1 percent of GDP which was contracted last September with a regional bank was about one point of percentage short of the 35 percent required by the program. As the loan was yet to be disbursed, they promptly contacted the lender upon being informed of this unintentional deviation from this program requirement, requesting an increase in the grant element of the loan to make it concessional in the program sense. Going forward, the authorities' intention to consult more closely with staff in similar situations will help avoid recurrence of this kind of incident.

13. In light of the authorities' strong commitment to the program and sound policy implementation, we would appreciate it if Directors would consider favorably today's request for completion of the fifth PSI and third ESF reviews. 


\section{INTERNATIONAL MONETARY FUND}

Public Information Notice (PIN) No. 10/65

FOR IMMEDIATE RELEASE

May 26, 2010
International Monetary Fund $70019^{\text {th }}$ Street, NW

Washington, DC 20431

USA

\section{IMF Executive Board Concludes 2010 Article IV Consultation with Senegal}

On May 24, 2010, the Executive Board of the International Monetary Fund (IMF) concluded the Article IV consultation with Senegal. ${ }^{1}$

\section{Background}

Following the food and fuel price shocks in 2008 , economic activity slowed further in 2009 because of the global economic downturn and domestic shocks, including temporary electricity shortages.

Real GDP growth is estimated to have been $1 \frac{1}{2}$ percent in 2009. Lower external demand and downward pressure on remittances, tourism receipts, and FDI have impacted growth. The agricultural sector, which benefited from supportive policies and favorable weather, helped to mitigate the impact of depressed demand at home and abroad on the secondary sector (construction, energy) and particularly the tertiary sector (transport, tourism services). The drop in crude oil and food prices pushed down consumer prices. Some fiscal easing has helped to cushion the impact of the global financial crisis. The overall fiscal deficit widened to 5.1 percent of GDP in 2009 from 3.7 percent of GDP two years earlier on account of lower tax revenues and higher expenditure. Inflation has been negative since the

\footnotetext{
${ }^{1}$ Under Article IV of the IMF's Articles of Agreement, the IMF holds discussions with each member, usually every year. A staff team visits the country, collects economic and financial information, and discusses with officials the country's economic developments and policies. On return to headquarters, the staff prepares a report, which forms the basis for discussion by the Executive Board. At the conclusion of the discussion, the Managing Director, as Chairman of the Board, summarizes the views of Executive Directors, and this summary is transmitted to the country's authorities.
} 
second half of 2009. The current account deficit declined mainly because of lower energy and food prices.

There are signs that Senegal's economy has started to recover. Growth is projected to reach $3 \frac{1}{2}$ percent in 2010 , driven by a pick-up in external demand and supportive domestic economic policies. Risks to the outlook relate to unexpectedly subdued external demand, financing constraints that limit the fiscal room for maneuver, and renewed problems with electricity supply. Opportunistic changes in economic policies for political reasons could also dampen prospects.

Senegal is pursuing its macroeconomic policies within an economic program supported by the IMF's Policy Support Instrument (PSI), which was approved in November 2007 (see Press Release No. 07/246). The authorities' program has four pillars: (i) containing the fiscal deficit to underpin macroeconomic stability and safeguard debt sustainability; (ii) improving fiscal governance and transparency so as to enhance policy credibility and sustain external assistance; (iii) encouraging private sector activity by improving the business environment and addressing structural impediments to economic growth and competitiveness; and (iv) limiting financial sector vulnerabilities and raising the sector's contribution to the economy.

\section{Executive Board Assessment}

Directors welcomed the broadly satisfactory implementation of the Senegalese authorities' economic program supported under the PSI and the ESF. However, a difficult external environment and domestic shocks, including in the energy sector, had slowed down the growth momentum. Directors noted that signs of a recovery have become visible and emphasized that prudent domestic macroeconomic policies and an accelerated implementation of structural reforms would help to strengthen growth further and reduce poverty.

While Senegal's risk of debt distress is low, Directors underscored the need to gradually withdraw the temporary fiscal stimulus and reduce the budget deficit to a level consistent with debt sustainability. They welcomed the authorities' plans to further strengthen revenue collection, which is already higher than the regional average, and encouraged the authorities to ensure that public entities pay their taxes on time. Directors stressed that spending pressures had to be contained to preserve macroeconomic stability and debt sustainability and meet the WAEMU convergence criteria, while safeguarding priority spending.

Directors welcomed the progress made by the authorities in eliminating payment delays to the private sector. They supported the authorities' commitment to finalize the full normalization of financial relations with the private sector by settling remaining extrabudgetary spending and public institution and agency debt through a transparent and publicly communicated process.

Directors supported efforts to reform public financial management and emphasized the need to maintain the reform momentum. They encouraged the authorities to improve their liquidity 
and debt management to complement the increasing integrity of their budget framework and expressed concern about program slippages that indicate that closer attention needs to be paid to spending procedures and control mechanism. Directors saw room for further strengthening the authorities' investment planning and evaluation with a view to ensuring high productivity of government spending.

While Senegal's real effective exchange rate has appreciated over the past years, Directors noted staff's assessment that it is not significantly overvalued. They underscored the need to overcome the weak export performance and to improve competitiveness through a more supportive business climate and better governance that would stimulate private-sector growth.

Directors underlined that other complementary policies need to be put in place to regain Senegal's growth momentum and return to previous growth trajectories. Sustained efforts are required to enhance the financial sector's contribution to the economy. Directors also encouraged the authorities to implement their energy sector reform plan to limit supply bottlenecks and fiscal risks.

Public Information Notices (PINs) form part of the IMF's efforts to promote transparency of the IMF's views and analysis of economic developments and policies. With the consent of the country (or countries) concerned, PINs are issued after Executive Board discussions of Article IV consultations with member countries, of its surveillance of developments at the regional level, of post-program monitoring, and of ex post assessments of member countries with longer-term program engagements. PINs are also issued after Executive Board discussions of general policy matters, unless otherwise decided by the Executive Board in a particular case. 
Senegal: Selected Economic Indicators

\begin{tabular}{|c|c|c|c|c|c|}
\hline & 2005 & 2006 & 2007 & 2008 & $\begin{array}{r}2009 \\
\text { Est. }\end{array}$ \\
\hline \multicolumn{6}{|l|}{ National income and prices (percent change) } \\
\hline GDP at constant prices & 5.6 & 2.4 & 4.8 & 2.3 & 1.5 \\
\hline Inflation (average) & 1.7 & 2.1 & 5.9 & 5.8 & -1.1 \\
\hline \multicolumn{6}{|l|}{ External sector } \\
\hline Current account balance (percent of GDP) & -9.0 & -9.5 & -11.8 & -14.3 & -8.7 \\
\hline Exports (in CFA francs, percent change) & 4.4 & 0.1 & -3.7 & 23.0 & -15.2 \\
\hline Imports (in CFA francs, percent change) & 15.6 & 9.6 & 19.5 & 25.8 & -20.4 \\
\hline Real effective exchange rate (percent change) & -1.0 & -0.2 & 5.3 & 4.4 & -1.7 \\
\hline \multicolumn{6}{|l|}{ Money and credit } \\
\hline Credit to the economy (percent change) & 24.5 & 4.2 & 10.5 & 17.2 & 3.6 \\
\hline \multicolumn{6}{|l|}{ Government budget (percent of GDP) } \\
\hline Revenue & 19.2 & 19.7 & 21.1 & 19.4 & 18.7 \\
\hline Grants & 1.6 & 1.5 & 2.6 & 2.4 & 3.0 \\
\hline Total expenditure and net lending & 24.0 & 27.2 & 27.6 & 26.6 & 27.1 \\
\hline Overall balance & -3.0 & -5.7 & -3.7 & -4.6 & -5.1 \\
\hline Central government domestic debt & 3.3 & 5.3 & 6.6 & 5.3 & 8.1 \\
\hline External public debt & 42.3 & 17.7 & 17.9 & 19.8 & 24.0 \\
\hline
\end{tabular}

Source: Senegalese authorities and IMF staff estimates. 